\title{
On conformal supergravity and harmonic superspace
}

\author{
Daniel Butter \\ Nikhef Theory Group, \\ Science Park 105, 1098 XG Amsterdam, The Netherlands \\ E-mail: dbutter@nikhef.nl
}

ABSTRACT: This paper describes a fully covariant approach to harmonic superspace. It is based on the conformal superspace description of conformal supergravity and involves extending the supermanifold $\mathcal{M}^{4 \mid 8}$ by the tangent bundle of $\mathbb{C} P^{1}$. The resulting superspace $\mathcal{M}^{4 \mid 8} \times T \mathbb{C} P^{1}$ can be identified in a certain gauge with the conventional harmonic superspace $\mathcal{M}^{4 \mid 8} \times S^{2}$. This approach not only makes the connection to projective superspace transparent, but simplifies calculations in harmonic superspace significantly by eliminating the need to deal directly with supergravity prepotentials. As an application of the covariant approach, we derive from harmonic superspace the full component action for the sigma model of a hyperkähler cone coupled to conformal supergravity. Further applications are also sketched.

Keywords: Extended Supersymmetry, Superspaces, Supergravity Models

ARXiv EPrint: 1508.07718 


\section{Contents}

1 Introduction $\quad 2$

2 Harmonic analysis on the complexified $S^{2} \quad 6$

2.1 Elements of analysis on a real $S^{2} \quad 6$

$\begin{array}{ll}2.2 & \text { Analysis on the complexified } S^{2} \text { and twisted biholomorphy }\end{array}$

2.3 The emergence of a complex harmonic structure 9

$\begin{array}{ll}2.4 \text { Complex harmonic integration } & 11\end{array}$

3 Complex harmonic superspace on $\mathcal{M}^{4 \mid 8} \times \mathrm{SU}(2)_{\mathrm{v}} \times \mathrm{SU}(2)_{\mathrm{w}} \quad 12$

$\begin{array}{lll}3.1 & \text { Construction in the central basis } & 13\end{array}$

$\begin{array}{lll}3.2 & \text { Covariant primary analytic superfields } & 16\end{array}$

4 Superspace action principles on $\mathcal{M}^{4 \mid 8} \times \mathrm{SU}(2)_{\mathrm{v}} \times \mathrm{SU}(2)_{\mathrm{w}} \quad 20$

$\begin{array}{lll}4.1 & \text { Full superspace } & 20\end{array}$

$\begin{array}{ll}4.2 \text { Analytic superspace } & 21\end{array}$

$\begin{array}{lll}4.3 & \text { Analytic superspace component action } & 22\end{array}$

4.4 Examples of complex harmonic superspace actions 25

5 The analytic basis and conventional harmonic superspace $\quad 27$

$\begin{array}{lll}5.1 & \text { Rigid harmonic superspace and its analytic basis } & 27\end{array}$

5.2 The analytic basis in complex harmonic superspace 30

$\begin{array}{lll}5.3 & \text { Curved harmonic superspace and analytic densities } & 32\end{array}$

5.4 Examples in the analytic basis 34

5.5 Harmonic superspace on $\mathcal{M}^{4 \mid 8} \times S^{2} \quad 35$

6 Superconformal sigma models from curved harmonic superspace $\quad 37$

6.1 Hyperkähler geometry and on-shell $\mathcal{N}=2$ superfields 38

6.2 The component fields of $Q^{\mathrm{a}+}$ and auxiliary equations of motion 43

6.3 Summary of the component calculation 44

$\begin{array}{lll}7 & \text { Further applications and outlook } & 48\end{array}$

A Analytic integrals and densities $\quad \mathbf{5 2}$

$\begin{array}{lll}\text { A.1 Analytic submanifolds } & 52\end{array}$

$\begin{array}{lll}\text { A.2 Analytic gauge, densities and transformation rules } & 54\end{array}$

A.3 Rules for total derivatives $\quad 55$ 


\section{Introduction}

$\mathcal{N}=2$ supersymmetric theories in four dimensions face a particular hurdle relative to their $\mathcal{N}=1$ cousins: the general matter hypermultiplet cannot be off-shell without introducing an infinite number of auxiliary fields. This understandably makes the direct construction of supersymmetric actions - a straightforward procedure for $\mathcal{N}=1$ actions even with supergravity couplings and higher derivatives - significantly more difficult as conventional $\mathcal{N}=2$ superspace proves insufficient. Instead, one requires a more elaborate superspace where infinite sets of auxiliary fields are encoded in a controlled way so that the most general off-shell actions of hypermultiplets and vector multiplets may be described. There are two well-developed options: harmonic superspace and projective superspace. Harmonic superspace, developed by Galperin, Ivanov, Kalitzin, Ogievetsky and Sokatchev [1, 2] exploits an additional bosonic manifold $S^{2}$, with the infinite auxiliary fields appearing in a convergent harmonic expansion. In contrast, the projective superspace approach, constructed by Karlhede, Lindström, and Roček [3-5] (see also the recent reviews [6, 7]) involves an auxiliary $\mathbb{C} P^{1}$ where the hypermultiplet is a holomorphic function near one of the poles, with the auxiliary fields described by coefficients in a Taylor expansion. ${ }^{1}$

While $S^{2}$ and $\mathbb{C} P^{1}$ describe the same manifold, the differing nature of the superfields has important consequences. For example, the respective action principles on the two spaces are quite different: harmonic actions involve integrals over the $S^{2}$ and are completely specified by their Lagrangians, while projective actions are defined on a contour in $\mathbb{C} P^{1}$, with different contours corresponding (in principle) to different actions for the same Lagrangian. But there are other differences between these two approaches which are less obviously connected with their auxiliary structures. Quite early on, prepotential superfields were identified within harmonic superspace both for gauge theories and supergravity [1]; these enabled a large body of supergraph calculations involving vector and hypermultiplets. In contrast, while projective prepotentials appeared in $[5]$ (see $[11,12]$ for a discussion of gauge prepotentials on curved supermanifolds) projective supergraph calculations in non-abelian gauge theories have appeared only relatively recently [13-17], while supergravity prepotentials remain terra incognita. This does not prevent the construction of supergravity actions in projective superspace; to the contrary, an extremely powerful manifestly covariant method has been developed over the last few years to address general supergravity-matter systems and their component reduction, first in five dimensions [18-20] and then in four [10, 21-23], building on the initial work of [24]. Within harmonic superspace, covariant methods have been explored in two papers $[25,26]$, which addressed how to derive supergravity prepotentials from a covariant supergeometry, but further applications of harmonic superspace to supergravity systems have mainly used prepotentials.

The distinction between a prepotential-based approach and a fully covariant method can be illustrated with a simple example. Take pure (gauged) supergravity consisting of a

\footnotetext{
${ }^{1}$ A general framework for discussing higher $\mathcal{N}$ analogues of harmonic and projective superspace involves the so-called $(\mathcal{N}, p, q)$ superspaces, introduced by Hartwell and Howe [8, 9]. These emphasize the geometrization of the $R$-symmetry group and the nature of the superconformal transformations, both of which play an important role here. As in [10], our discussion corresponds to the case $(2,1,1)$.
} 
single vector multiplet compensator coupled to a single hypermultiplet compensator. Using the arctic multiplet $\Upsilon^{+}$of projective superspace as the hypermultiplet, the action reads ${ }^{2}$

$$
S=-\frac{1}{4} \int \mathrm{d}^{4} x \mathrm{~d}^{4} \theta \mathcal{E} W^{2}+\frac{1}{2 \pi} \oint_{\mathcal{C}} \mathrm{d} \tau \int \mathrm{d}^{4} x \mathrm{~d}^{4} \theta^{+} \mathcal{E}^{--}\left(2 i \breve{\Upsilon}^{+} \Upsilon^{+}\right)
$$

The arctic multiplets in the second term carry charge $g$ under the vector multiplet; if the vector prepotential were made explicit, this term would be written $\breve{\Upsilon}^{+} e^{g V} \Upsilon^{+}$. The important feature is that both terms above are manifestly covariant and defined in any gauge. In particular, one can make arbitrary conformal supergravity gauge transformations (with arbitrary superfield parameters) for both actions, and invariance is ensured using the properties of the respective chiral and analytic measures $\mathcal{E}$ and $\mathcal{E}^{--}$[10]. In harmonic superspace, the corresponding action involving the harmonic hypermultiplet $\widehat{Q}^{+}$is rendered with explicit prepotentials as

$$
S=-\frac{1}{4} \int \mathrm{d} \hat{u} \mathrm{~d}^{4} \hat{x} \mathrm{~d}^{8} \hat{\theta} \hat{E} V^{++} V^{--}+2 \int \mathrm{d} \hat{u} \mathrm{~d}^{4} \hat{x} \mathrm{~d}^{4} \hat{\theta}^{+}\left(\widetilde{\widehat{Q}}^{+}\left(\hat{\mathcal{D}}^{++}-i g V^{++}\right) \widehat{Q}^{+}\right) .
$$

One works in the analytic basis for the coordinates, which we have denoted with hats. In this gauge, explicit gravitational prepotentials appear within the harmonic covariant derivative $\hat{\mathcal{D}}^{++}$; these can in turn be used to construct the full superspace measure in the analytic basis, denoted $\hat{E}$ above. There is no analytic measure as $\widehat{Q}^{+}$is chosen to transform as a scalar density under analytic diffeomorphisms.

The advantage of the second action, and a prepotential approach in particular, is the relative ease with which one can calculate superspace equations of motion and perform quantum calculations. These were major successes of the harmonic approach, and require the dependence on the prepotentials to be laid bare; that dependence is obscured in a covariant formulation. On the other hand, there are a number of advantages of a covariant formulation. The first action (1.1) is constructed in a generic gauge, and its component reduction can be performed in a manifestly covariant manner (see e.g. [27, 28] for the two pieces). To reduce the second action (1.2) to components, one must adopt a Wess-Zumino gauge for the various analytic prepotentials, perform the $\theta$ integrals, and then reconstitute various composite objects such as the covariant derivative, the spin connection, etc. While this is possible in principle, to our knowledge it has never been explicitly undertaken for all terms in any supergravity action; even the most extensive component treatment [29] of general supergravity-matter actions in harmonic superspace restricted to bosonic terms.

Of course, for actions like those discussed above, the question of how easy it is to perform a component reduction is essentially moot, as the results are well-known. A more interesting question is how to construct new higher-derivative actions for hypermultiplets coupled to supergravity, and to analyze the dependence of such higher derivative terms on the underlying prepotentials so that one may analyze supersymmetric equations of motion, supercurrents, and so forth. For addressing such questions, it is useful to have a formulation of general supergravity-matter systems with both a covariant and a prepotential description.

\footnotetext{
${ }^{2}$ The projective action for an arctic multiplet minimally coupled to a vector multiplet and conformal supergravity first appeared in 5D [20] where a different but equivalent action principle was employed. We are using here the reformulation of projective superspace given in [10].
} 
Our major goal in this paper will be to provide a covariant reformulation of supergravity-matter actions in harmonic superspace so that any action, even a higherderivative one, can be addressed in a manifestly covariant way. Because the prepotential approach for harmonic superspace already exists, we will begin by seeking a manifestly covariant formulation from the outset. This will cover some similar ground as [25, 26], but where these authors were concerned with Einstein supergravity (with two hidden implicit compensators within the supergeometry), we will build conformal supergravity into the structure group of superspace from the very beginning. This so-called conformal superspace approach, which corresponds to the superconformal tensor calculus in components, offers significant simplifications to calculations: recent applications have included constructing previously unknown higher-derivative invariants in $5 \mathrm{D}$ as well as the construction of all off-shell 3D $\mathcal{N} \leq 6$ conformal supergravity actions, including auxiliary fields [30, 31]. ${ }^{3}$ In applying this to harmonic superspace, it will permit us to give not only covariant reformulations of all harmonic superspace actions, but will also allow the construction of the covariant component reduction rule for a general analytic Lagrangian - novel results not found in any previous formulation of harmonic superspace.

Interestingly, the incorporation of the superconformal algebra directly into the structure group, and the presence of the $\mathrm{SU}(2) R$-symmetry group in particular, will necessitate a reinterpretation of just what harmonic superspace actually is. Before elaborating further, we should pause to answer the following question: just why should one focus on harmonic superspace when another formulation - projective superspace - already offers fully developed covariant methods? The answer is that projective and harmonic superspace are actually not intrinsically different approaches, but possess a quite non-trivial relation. By fully addressing these issues within harmonic superspace — in part using inspiration from projective superspace - we can learn important lessons about both. Recently, Jain and Siegel have argued to interpret projective superspace as an analytic continuation of harmonic superspace [33]. ${ }^{4}$ This has proven to be a robust scheme and has enabled a direct link between the harmonic and projective descriptions of vector prepotentials [33] and between their respective descriptions of hyperkähler sigma models [36]. The main idea is to "complexify" the $S^{2}$ of harmonic superspace to the tangent bundle of $\mathbb{C} P^{1}$, identified as $\mathbb{C} P^{1} \times \mathbb{C} P^{1}$ with the anti-diagonal removed. The two $\mathbb{C} P^{1}$ factors possess different $\mathrm{SU}(2)$ isometry groups. The first is to be identified with the $R$-symmetry subgroup of the superconformal group, while the second is a spectator. The connection between projective and harmonic superfields can be described concisely as follows:

- The natural superfields on (complex) harmonic superspace are biharmonic functions $\mathcal{F}^{(n, m)}$ with charge $(n, m)$ under the $\mathrm{U}(1)_{\mathrm{v}} \times \mathrm{U}(1)_{\mathrm{w}}$ subgroup of $\mathrm{SU}(2)_{\mathrm{v}} \times \mathrm{SU}(2)_{\mathrm{w}}$ of

\footnotetext{
${ }^{3}$ The $3 \mathrm{D} \mathcal{N} \leq 5$ component actions were constructed for the first time using superspace techniques. The $\mathcal{N}=6$ action was constructed first in components [32] and then in superspace.

${ }^{4}$ This analytic continuation is related to a similar approach in twistor theory [34]. An earlier proposal by Kuzenko [35] to relate harmonic and projective superspace works rather differently.
} 
the form (for $n+m \geq 0$ )

$$
\mathcal{F}^{(n, m)}=\left(v^{+}, w^{-}\right)^{n} \sum_{k=0}^{\infty} \mathcal{F}^{\left(i_{1} \cdots i_{n+m+k} j_{1} \cdots j_{k}\right)} \frac{v_{i_{1}}^{+}}{\left(v^{+}, w^{-}\right)} \cdots \frac{v_{i_{n+m+k}}^{+}}{\left(v^{+}, w^{-}\right)} w_{j_{1}}^{-} \cdots w_{j_{k}}^{-} .
$$

(A similar series exists for $n+m<0$.) The series $(1.3)$ is presumed to converge on the so-called real $S^{2}$ manifold, corresponding to the diagonal submanifold of $\mathbb{C} P^{1} \times \mathbb{C} P^{1}$ where $v^{i+} \propto w^{i+}$. The anti-diagonal subset of $\mathbb{C} P^{1} \times \mathbb{C} P^{1}$ where $v^{i+} \propto w^{i-}$ must be excluded so that the series exists (at least asymptotically) everywhere; this implies that we are dealing with the tangent bundle of $\mathbb{C} P^{1}$.

- Associated with every biharmonic function $\mathcal{F}^{(n, m)}$ is an arctic superfield $\Upsilon^{(n)}$ and an antarctic superfield $\breve{\Upsilon}^{(n)}$ given by

$$
\begin{aligned}
& \Upsilon^{(n)}=\left.\mathcal{F}^{(n, m)}\right|_{w_{i}^{-}=(1,0)}=\left(v^{\underline{1}}\right)^{n} \sum_{j=0}^{\infty} \Upsilon_{j} \zeta^{j}, \\
& \breve{\Upsilon}^{(n)}=\left.\mathcal{F}^{(n, m)}\right|_{w_{i}^{-}=(0,1)}=\left(v^{\underline{2}}\right)^{n} \sum_{j=0}^{\infty} \tilde{\Upsilon}_{j}(-\zeta)^{-j} .
\end{aligned}
$$

The arctic nature of $\Upsilon^{(n)}$ and the antarctic nature of $\breve{\Upsilon \Upsilon}^{(n)}$ are guaranteed because of the presumed convergence of (1.3) in the vicinity of $S^{2}$.

This interpretation of harmonic superspace is actually not particularly revolutionary. As discussed in the original harmonic superspace literature [37], the superconformal group acts on complex harmonics $u^{i \pm}$, but one performs the harmonic integrals as if they were real. This suggests (see the comment in chapter 9 of [2]) that the harmonic $S^{2}$ should be reinterpreted as lying within $\mathbb{C} P^{1} \times \mathbb{C} P^{1}$. Guided by these old observations and the requirement to reproduce the harmonic-projective mapping in curved space, a covariant scheme immediately presents itself. We will begin with the covariant projective superspace of [10] defined on the supermanifold $\mathcal{M}^{4 \mid 8} \times \mathrm{SU}(2)$. (As fields and operators had fixed charges in $\mathrm{U}(1) \subset \mathrm{SU}(2)$, this effectively became $\mathcal{M}^{4 \mid 8} \times \mathbb{C} P^{1}$.) The auxiliary $\mathrm{SU}(2)$ was identified with the $\mathrm{SU}(2) R$-symmetry group and non-trivial $R$-symmetry curvature was encoded in the fibering of the $\mathrm{SU}(2)$ over $\mathcal{M}^{4 \mid 8}$. We then extend the auxiliary manifold with an additional completely rigid $\mathrm{SU}(2)$ factor, giving the supermanifold $\mathcal{M}^{4 \mid 8} \times \mathrm{SU}(2) \times$ $\mathrm{SU}(2)$ (effectively $\mathcal{M}^{4 \mid 8} \times T \mathbb{C} P^{1}$ ). Over this complex harmonic manifold, we will introduce superfields defined exactly as in the rigid case sketched above. The virtue of this approach is that it efficiently meets two goals. First, it gives a covariant formulation that agrees (as we will show) with the conventional harmonic superspace description whose harmonics are naturally complex but integrated on a real $S^{2}$. Second, it permits the mapping between harmonic and projective superspace to be lifted to a general curved supermanifold.

To describe the biharmonic space, we will need two sets of harmonics $v^{i \pm}$ and $w^{i \pm}$, or equivalently, complex harmonics $u^{i \pm}$ and additional coordinates $z^{ \pm \pm}$and $z^{0}$. This complex harmonic description has already been employed within the harmonic superspace literature to describe the target space of quaternionic sigma models, where some of the harmonics are 
interpreted as compensator fields for the sigma model [38]. We will be using these complex harmonics to instead describe the auxiliary harmonic manifold of superspace itself. Once this point of view is adopted, we will see that many curious features of the conventional harmonic approach reveal themselves quite naturally.

It is worth observing that biprojective superfields have already been discussed in [3941] to describe extended supersymmetric systems in two dimensions (see also [42, 43] for curved superspace applications of biprojective superfields in $2 \mathrm{D}$ and $3 \mathrm{D})$. In these cases, the $R$-symmetry group is $\mathrm{SU}(2) \times \mathrm{SU}(2)$, and so conventional projective and harmonic approaches already lead to $\mathbb{C} P^{1} \times \mathbb{C} P^{1}$. Constructing a complex harmonic superspace for these cases would seem to lead to a quadriharmonic space involving $\left(\mathbb{C} P^{1}\right)^{4}$.

This paper is organized as follows. In section 2, we review some details of harmonic analysis on $T \mathbb{C} P^{1}$. Our approach will use some of the tools and ideas introduced in [38], but our conventions and emphasis will differ rather extensively. In section 3 , we present a concise discussion of the covariant harmonic superspace $\mathcal{M}^{4 \mid 8} \times \mathrm{SU}(2) \times \mathrm{SU}(2)$ built on the covariant projective superspace $\mathcal{M}^{4 \mid 8} \times \mathrm{SU}(2)$. Covariant action principles, including the covariant component reduction, will be discussed in section 4 . These two sections establish the self-consistency of our approach. Section 5 is somewhat disconnected and may be omitted for those interested only in the covariant superconformal approach: there we show that this formulation agrees with the conventional harmonic superspace in the analytic basis, and we relate it to the existing covariant approach of $[25,26]$.

The main applications are contained within the last two sections. As a sample calculation, we demonstrate in section 6 the covariant component reduction of a general superconformal sigma model, reproducing the general hyperkähler cone sigma model coupled to conformal supergravity [44] just as in projective superspace [27]. The result of this calculation is not new, but it provides a useful test that covariant component reductions within harmonic superspace are tractable. Our interest is actually in exploring higher-derivative actions. A brief discussion of these applications follows in the concluding section. Our notation and conventions follow [10]. A technical appendix addresses aspects of integration on analytic submanifolds, to which we will refer as needed.

\section{Harmonic analysis on the complexified $S^{2}$}

We begin with a discussion of elements of harmonic analysis on the complexified $S^{2}$, which is equivalent to $T \mathbb{C} P^{1}$. The formulation uses the biharmonic approach of [38], although we will use somewhat different conventions and emphasize different aspects.

\subsection{Elements of analysis on a real $S^{2}$}

Let us briefly review the harmonic description of an $S^{2}$ manifold [2]. It is described by harmonics $u^{i+}$ and $u_{i}^{-}$obeying $u_{i}^{-}=\left(u^{i+}\right)^{*}$ with $u^{i+} u_{i}^{-}=1$. These parametrize a group element $\mathbf{g}$ of $\mathrm{SU}(2)$,

$$
\mathbf{g}=\left(\begin{array}{cc}
u^{\underline{1}+} & -u_{\underline{2}}^{-} \\
u^{\underline{2}} & u_{\underline{1}}^{-}
\end{array}\right), \quad \mathbf{g}^{-1}=\mathbf{g}^{\dagger}, \quad \operatorname{det} \mathbf{g}=1
$$


The two-sphere is isomorphic to $\mathrm{SU}(2) / \mathrm{U}(1)$ with the equivalence relation $u^{i+} \sim e^{i \alpha} u^{i+}$. That is, the harmonics are in one-to-one correspondence with real coordinates $X^{I}=$ $u_{j}^{-}\left(\sigma^{I}\right)^{j}{ }_{k} u^{k+}$ obeying $\sum_{I} X^{I} X^{I}=1$, where $\sigma^{I}$ are the Pauli matrices.

Rather than introduce two derivatives and one tangent space rotation on $S^{2}$, it is customary to introduce three $\mathrm{SU}(2)$ derivatives, $D_{\mathrm{u}}^{++}, D_{\mathrm{u}}^{0}$ and $D_{\mathrm{u}}^{--}$defined as

$$
D_{\mathrm{u}}^{++} \equiv u_{i}^{+} \frac{\partial}{\partial u_{i}^{-}}, \quad D_{\mathrm{u}}^{--} \equiv u^{i-} \frac{\partial}{\partial u^{i+}}, \quad D_{\mathrm{u}}^{0} \equiv u^{i+} \frac{\partial}{\partial u^{i+}}-u_{i}^{-} \frac{\partial}{\partial u_{i}^{-}},
$$

possessing the commutation relations $\left[D_{\mathrm{u}}^{++}, D_{\mathrm{u}}^{--}\right]=D_{\mathrm{u}}^{0}$ and $\left[D_{\mathrm{u}}^{0}, D_{\mathrm{u}}^{ \pm \pm}\right]= \pm 2 D_{\mathrm{u}}^{ \pm \pm}$. Associated with these are vielbeins

$$
\mathcal{U}^{++}=u_{i}^{+} \mathrm{d} u^{i+}, \quad \mathcal{U}^{--}=u_{i}^{-} \mathrm{d} u^{i-}, \quad \mathcal{U}^{0}=u_{i}^{-} \mathrm{d} u^{i+}=u_{i}^{+} \mathrm{d} u^{i-},
$$

so that the exterior derivative can be written $\mathrm{d}=-\mathcal{U}^{++} D_{\mathrm{u}}^{--}+\mathcal{U}^{0} D_{\mathrm{u}}^{0}+\mathcal{U}^{--} D_{\mathrm{u}}^{++}$. We employ the usual superspace conventions for differential forms so that the exterior derivative acts from the right.

Given some globally defined function $f^{(0)}\left(u^{+}, u^{-}\right)$, its integral on $S^{2}$ is given by

$$
\int_{S^{2}} \mathrm{~d} u f^{(0)}=\frac{i}{2 \pi} \int_{S^{2}} \mathcal{U}^{++} \wedge \mathcal{U}^{--} f^{(0)}
$$

normalized so that $\int_{S^{2}} \mathrm{~d} u=\frac{i}{2 \pi} \int_{S^{2}} \mathcal{U}^{++} \wedge \mathcal{U}^{--}=1$. The integrand can be interpreted as a closed two-form $\omega=\mathcal{U}^{++} \wedge \mathcal{U}^{--} f^{(0)}$ on either $S^{2}$ or $\mathrm{SU}(2)$.

\subsection{Analysis on the complexified $S^{2}$ and twisted biholomorphy}

We define the complexified $S^{2}$ as the complex affine quadric $Q^{2}$,

$$
Q^{2}=\left\{Z^{I} \in \mathbb{C}^{3}: \sum_{I=1}^{3}\left(Z^{I}\right)^{2}=1\right\}
$$

One can show that $Q^{2} \subset \mathbb{C} P^{1} \times \mathbb{C} P^{1}$ by identifying

$$
Z^{I}=\frac{1}{(v, \bar{w})} \bar{w}_{j}\left(\sigma^{I}\right)_{k}^{j} v^{k}, \quad(v, \bar{w}):=v^{k} \bar{w}_{k}
$$

which defines $v^{i}$ and $\bar{w}_{j}$ up to the identifications $v^{i} \sim \lambda v^{i}$ and $\bar{w}_{j} \sim \widetilde{\lambda}_{j}$ for $\lambda$ and $\tilde{\lambda}$ unrelated complex numbers. In other words, $Q^{2}$ can be identified as $\mathbb{C} P^{1} \times \mathbb{C} P^{1}$ with the anti-diagonal region $(v, \bar{w})=0$ excised: this is just the tangent bundle of $S^{2}{ }^{5}$

As with the real $S^{2}$, it is convenient to identify each $\mathbb{C} P^{1}$ with $\mathrm{SU}(2) / \mathrm{U}(1)$ and to introduce harmonic coordinates on the respective $\mathrm{SU}(2)$ groups. Denote the two groups by $\mathrm{SU}(2)_{\mathrm{v}}$ and $\mathrm{SU}(2)_{\mathrm{w}}$ with harmonics $v^{i \pm}$ and $w^{i \pm}$ defined as

$$
v^{i+}=\frac{v^{i}}{\sqrt{(v, \bar{v})}}, \quad v_{i}^{-}=\frac{\bar{v}_{i}}{\sqrt{(v, \bar{v})}},
$$

\footnotetext{
${ }^{5}$ One can prove $Q^{2} \cong T \mathbb{C} P^{1}$ directly by decomposing $Z^{I}$ into its real and imaginary parts.
} 
and similarly for $w^{i \pm}$. The corresponding derivatives $\left(D_{\mathrm{v}}^{ \pm \pm}, D_{\mathrm{v}}^{0}\right)$ and $\left(D_{\mathrm{w}}^{ \pm \pm}, D_{\mathrm{w}}^{0}\right)$, as well as the vielbeins $\left(\mathcal{V}^{ \pm \pm}, \mathcal{V}^{0}\right)$ and $\left(\mathcal{W}^{ \pm \pm}, \mathcal{W}^{0}\right)$, are defined analogously to $(2.2)$ and (2.3). We write the exterior derivative as $\mathrm{d}=\mathcal{V}^{\underline{a}} D_{\mathrm{v} \underline{a}}+\mathcal{W}^{\bar{a}} D_{\mathrm{w} \bar{a}}$ where

$$
\mathcal{V}^{\underline{a}} D_{\mathrm{v} \underline{a}}=-\mathcal{V}^{++} D_{\mathrm{v}}^{--}+\mathcal{V}^{0} D_{\mathrm{v}}^{0}+\mathcal{V}^{--} D_{\mathrm{v}}^{++}=\mathcal{V}^{++} D_{\mathrm{v}++}+\mathcal{V}^{0} D_{\mathrm{v} 0}+\mathcal{V}^{--} D_{\mathrm{v}--}
$$

and similarly for $\mathcal{W}^{\bar{a}} D_{\mathrm{w} \bar{a}}$.

The space $Q^{2} \cong T \mathbb{C} P^{1}$ is defined above in a twisted biholomorphic manner - that is, the coordinates $Z^{I}$ are holomorphic in $v^{i}$ and anti-holomorphic in $\bar{w}_{i}$. We will be mainly interested in fields that share this feature, properly interpreted on the harmonic coordinates. Following the same abuse of nomenclature as in [10], we will refer to fields annihilated by $D_{\mathrm{v}}^{++}$as holomorphic on (an open domain of) $\mathrm{SU}(2)_{\mathrm{v}}$ and those annihilated by $D_{\mathrm{w}}^{--}$as anti-holomorphic on (an open domain of) $\mathrm{SU}(2)_{\mathrm{w}}$. Fields satisfying both conditions will be called (twisted) biholomorphic. We specialize to such fields $\mathcal{F}^{(n, m)}$ with charge $(n, m)$ under $\mathrm{U}(1)_{\mathrm{v}} \times \mathrm{U}(1)_{\mathrm{w}}$, so that

$$
D_{\mathrm{v}}^{0} \mathcal{F}^{(n, m)}=n \mathcal{F}^{(n, m)}, \quad D_{\mathrm{w}}^{0} \mathcal{F}^{(n, m)}=m \mathcal{F}^{(n, m)}, \quad D_{\mathrm{v}}^{++} \mathcal{F}^{(n, m)}=D_{\mathrm{w}}^{--} \mathcal{F}^{(n, m)}=0 .
$$

The natural integration principle is a twisted biholomorphic integral

$$
S=\frac{i}{2 \pi} \int_{\mathcal{S}} \mathcal{V}^{++} \wedge \mathcal{W}^{--} \omega^{(-2,+2)}\left(v^{+}, w^{-}\right)=\frac{i}{2 \pi} \int_{\mathcal{S}} \omega
$$

where $\omega$ is a two-form and $\mathcal{S}$ is some closed two-dimensional surface in $T \mathbb{C} P^{1} . \omega$ is closed as a consequence of being twisted biholomorphic. The action is invariant under an infinitesimal diffeomorphism $\delta_{\xi} \omega=\mathrm{d}\left(\imath_{\xi} \omega\right)$ corresponding to a small deformation of the surface $\mathcal{S}$, and so it depends only on the homotopy class of the surface provided $\omega$ is non-singular in the interior. In fact, as we will discuss shortly, there is but one interesting class for $\mathcal{S}$.

We will actually need a slightly more general two-form given by

$$
\omega=\mathcal{V}^{++} \wedge \mathcal{W}^{--} \omega^{(-2,2)}-\mathcal{V}^{--} \wedge \mathcal{W}^{--} \omega^{(2,2)}
$$

In this case, the closure condition amounts to two requirements. The first is

$$
D_{\mathrm{w}}^{--} \omega^{(-2,2)}=0, \quad D_{\mathrm{w}}^{--} \omega^{(2,2)}=0,
$$

equivalent to the condition that $\omega$ is anti-holomorphic in $w_{i}^{-}$. The second requirement

$$
D_{\mathrm{v}}^{++} \omega^{(-2,2)}=D_{\mathrm{v}}^{--} \omega^{(2,2)},
$$

constrains the $v_{i}^{-}$dependence of $\omega^{(-2,2)}$.

For later use, it will be convenient to establish two analogues of Stokes' formula. Taking $\Lambda^{(0,2)}$ and $\Lambda^{(-2,0)}$ to be functions annihilated by $D_{\mathrm{w}}^{--}$but otherwise arbitrary, one can show

$$
\int_{\mathcal{S}} \mathcal{V}^{++} \wedge \mathcal{W}^{--} D_{\mathrm{v}}^{--} \Lambda^{(0,2)}=\int_{\mathcal{S}} \mathcal{V}^{--} \wedge \mathcal{W}^{--} D_{\mathrm{v}}^{++} \Lambda^{(0,2)}, \quad D_{\mathrm{w}}^{--} \Lambda^{(0,2)}=0
$$




$$
\int_{\mathcal{S}} \mathcal{V}^{++} \wedge \mathcal{W}^{--} D_{\mathrm{w}}^{++} \Lambda^{(-2,0)}=-\int_{\mathcal{S}} \mathcal{V}^{++} \wedge \mathcal{V}^{--} D_{\mathrm{v}}^{++} \Lambda^{(-2,0)}, \quad D_{\mathrm{w}}^{--} \Lambda^{(-2,0)}=0
$$

An important special case is when $\Lambda^{(0,2)}$ and $\Lambda^{(-2,0)}$ are twisted biholomorphic and then the right-hand sides of (2.14) vanish.

A special class of diffeomorphisms are the isometries that leave the vielbeins invariant. $\mathrm{An} \mathrm{SU}(2)_{\mathrm{v}}$ isometry acts as

$$
\delta_{\mathrm{v}}(\lambda)=-\lambda_{\mathrm{v}}^{++} D_{\mathrm{v}}^{--}+\lambda_{\mathrm{v}}^{0} D_{\mathrm{v}}^{0}+\lambda_{\mathrm{v}}^{--} D_{\mathrm{v}}^{++}, \quad \lambda_{\mathrm{v}}^{ \pm \pm}=\lambda^{i j} v_{i}^{ \pm} v_{j}^{ \pm}, \quad \lambda_{\mathrm{v}}^{0}=\lambda^{i j} v_{i}^{+} v_{j}^{-}
$$

in terms of harmonic-independent $\lambda^{i j}$. Similar formulae hold for an $\operatorname{SU}(2)_{\mathrm{w}}$ isometry $\delta_{\mathrm{w}}(\rho)$. It is interesting to note for these isometries that

$$
\begin{aligned}
\delta_{\mathrm{v}} \omega^{(-2,2)} & =D_{\mathrm{v}}^{--}\left(\lambda_{\mathrm{v}}^{--} \omega^{(2,2)}-\lambda_{\mathrm{v}}^{++} \omega^{(-2,2)}\right), & \delta_{\mathrm{w}} \omega^{(-2,2)} & =D_{\mathrm{w}}^{++}\left(\rho_{\mathrm{w}}^{--} \omega^{(-2,2)}\right), \\
\delta_{\mathrm{v}} \omega^{(2,2)} & =D_{\mathrm{v}}^{++}\left(\lambda_{\mathrm{v}}^{--} \omega^{(2,2)}-\lambda_{\mathrm{v}}^{++} \omega^{(-2,2)}\right), & \delta_{\mathrm{w}} \omega^{(2,2)} & =D_{\mathrm{w}}^{++}\left(\rho_{\mathrm{w}}^{--} \omega^{(2,2)}\right) .
\end{aligned}
$$

\subsection{The emergence of a complex harmonic structure}

Now let us recover the complex harmonic structure required for harmonic superspace. The simplest choice of closed surface $\mathcal{S}$ is the real $S^{2}=\mathrm{SU}(2) / \mathrm{U}(1)$ constructed from the diagonal $\mathrm{SU}(2)$ submanifold of $\mathrm{SU}(2)_{\mathrm{v}} \times \mathrm{SU}(2)_{\mathrm{w}}$ with $v^{i \pm}=w^{i \pm}$. Up to small deformations this is the only homotopically non-trivial choice: any non-contractible $\mathcal{S}$ in $T \mathbb{C} P^{1}$ is continuously deformable into the real $S^{2}$. We require an additional assumption that each of the twisted biholomorphic quantities are globally defined along this submanifold. This implies that $\mathcal{F}^{(n, m)}$ possesses an expansion as in [38]

$$
\mathcal{F}^{(n, m)}=\left(v^{+}, w^{-}\right)^{n} \sum_{k=0}^{\infty} \mathcal{F}^{\left(i_{1} \cdots i_{n+m+k} j_{1} \cdots j_{k}\right)} \frac{v_{i_{1}}^{+}}{\left(v^{+}, w^{-}\right)} \cdots \frac{v_{i_{n+m+k}}^{+}}{\left(v^{+}, w^{-}\right)} w_{j_{1}}^{-} \cdots w_{j_{k}}^{-} .
$$

A similar expansion applies for $n+m<0$. To shed some light on the meaning of this, we follow [38] and introduce new complex harmonics,

$$
u_{i}^{+} \equiv u_{i}^{(0,1)}=\frac{v_{i}^{+}}{\left(v^{+}, w^{-}\right)}, \quad u_{i}^{-} \equiv u_{i}^{(0,-1)}=w_{i}^{-}, \quad u^{i+} u_{i}^{-}=1,
$$

and three additional complex coordinates,

$$
\begin{aligned}
z^{++} & \equiv z^{(0,2)}=\frac{\left(v^{+}, w^{+}\right)}{\left(v^{+}, w^{-}\right)} \\
z^{--} & \equiv z^{(0,-2)}=\left(v^{-}, w^{-}\right)\left(v^{+}, w^{-}\right) \\
z^{0} & \equiv z^{(1,-1)}=\left(v^{+}, w^{-}\right)
\end{aligned}
$$

We have followed existing convention in labeling the coordinates by the sums of their $\mathrm{U}(1)_{\mathrm{v}}$ and $\mathrm{U}(1)_{\mathrm{w}}$ charges. Note in particular that the complex harmonics and the coordinates $z^{ \pm \pm}$carry only $\mathrm{U}(1)_{\mathrm{w}}$ charge. Relative to the conventions of [38], we have exchanged the roles of $u^{i \pm}$ and $w^{i \pm}$ so that $u^{i \pm}$ is reserved for the complex harmonic coordinate. 
Now the original harmonics are given in terms of the new coordinates as

$$
v^{i+}=z^{0} u^{i+}, \quad v_{i}^{-}=\frac{1}{z^{0}}\left(u_{i}^{-}+u_{i}^{+} z^{--}\right), \quad w^{i+}=u^{i+}+z^{++} u^{i-}, \quad w_{i}^{-}=u_{i}^{-} .
$$

In the new variables, the old derivatives become

$$
\begin{aligned}
& D_{\mathrm{v}}^{++}=\left(z^{0}\right)^{2} \frac{\partial}{\partial z^{--}}, \quad D_{\mathrm{v}}^{0}=z^{0} \frac{\partial}{\partial z^{0}} \\
& D_{\mathrm{v}}^{--}=\frac{1}{\left(z^{0}\right)^{2}}\left(\partial_{\mathrm{u}}^{--}-\frac{\partial}{\partial z^{++}}+z^{--} z^{0} \frac{\partial}{\partial z^{0}}+\left(z^{--}\right)^{2} \frac{\partial}{\partial z^{--}}\right),
\end{aligned}
$$

and

$$
\begin{aligned}
D_{\mathrm{w}}^{++} & =\partial_{\mathrm{u}}^{++}-z^{++} \partial_{\mathrm{u}}^{0}-\left(z^{++}\right)^{2} \frac{\partial}{\partial z^{++}}+z^{++} z^{0} \frac{\partial}{\partial z^{0}}+\left(2 z^{++} z^{--}-1\right) \frac{\partial}{\partial z^{--}}, \\
D_{\mathrm{w}}^{0} & =\partial_{\mathrm{u}}^{0}+2 z^{++} \partial_{z^{++}}-2 z^{--} \partial_{z^{--}}-z^{0} \frac{\partial}{\partial z^{0}}, \quad D_{\mathrm{w}}^{--}=\frac{\partial}{\partial z^{++}} .
\end{aligned}
$$

The corresponding vielbeins are

$$
\begin{aligned}
\mathcal{V}^{++} & =\left(z^{0}\right)^{2} \mathcal{U}^{++}, \quad \mathcal{V}^{0}=\mathcal{U}^{0}+z^{--} \mathcal{U}^{++}+\frac{\mathrm{d} z^{0}}{z^{0}} \\
\mathcal{V}^{--} & =\frac{1}{\left(z^{0}\right)^{2}}\left(\mathcal{U}^{--}+2 z^{--} \mathcal{U}^{0}+\left(z^{--}\right)^{2} \mathcal{U}^{++}+\mathrm{d} z^{--}\right) \\
\mathcal{W}^{++} & =\mathcal{U}^{++}+2 z^{++} \mathcal{U}^{0}+\left(z^{++}\right)^{2} \mathcal{U}^{--}-\mathrm{d} z^{++} \\
\mathcal{W}^{0} & =\mathcal{U}^{0}+z^{++} \mathcal{U}^{--}, \quad \mathcal{W}^{--}=\mathcal{U}^{--} .
\end{aligned}
$$

We have introduced

$$
\begin{aligned}
& \partial_{\mathrm{u}}^{++}=u_{i}^{+} \frac{\partial}{\partial u_{i}^{-}}, \quad \partial_{\mathrm{u}}^{--}=u^{i-} \frac{\partial}{\partial u^{i+}}, \quad \partial_{\mathrm{u}}^{0}=u^{i+} \frac{\partial}{\partial u^{i+}}-u_{i}^{-} \frac{\partial}{\partial u_{i}^{-}}, \\
& \mathcal{U}^{++}=u_{i}^{+} \mathrm{d} u^{i+}, \quad \mathcal{U}^{--}=u_{i}^{-} \mathrm{d} u^{i-}, \quad \mathcal{U}^{0}=u_{i}^{-} \mathrm{d} u^{i+}=u_{i}^{+} \mathrm{d} u^{i-} .
\end{aligned}
$$

The $\mathrm{SU}(2)_{\mathrm{v}} \times \mathrm{SU}(2)_{\mathrm{w}}$ isometry transformations can be rewritten

$$
\begin{aligned}
& \delta_{\mathrm{v}}(\lambda)=\lambda_{\mathrm{u}}^{++}\left(\partial_{z^{++}}-\partial_{\mathrm{u}}^{--}\right)+\lambda_{\mathrm{u}}^{0}\left(z^{0} \partial_{z^{0}}+2 z^{--} \partial_{z^{--}}\right)+\lambda_{\mathrm{u}}^{--} \partial_{z^{--}}, \\
& \delta_{\mathrm{w}}(\rho)=-\rho_{\mathrm{u}}^{++} \partial_{z^{++}}+\rho_{\mathrm{u}}^{0}\left(\partial_{\mathrm{u}}^{0}-z^{0} \partial_{z^{0}}-2 z^{--} \partial_{z^{--}}\right)+\rho_{\mathrm{u}}^{--}\left(\partial_{\mathrm{u}}^{++}-\partial_{z^{--}}\right),
\end{aligned}
$$

where $\lambda_{\mathrm{u}}^{ \pm \pm}=\lambda^{i j} u_{i}^{ \pm} u_{j}^{ \pm}$and similarly for $\rho$. These special diffeomorphisms are induced by infinitesimal general coordinate transformations ${ }^{6}$

$$
\begin{aligned}
\delta_{\mathrm{v}}^{*} u^{i+} & =\lambda_{\mathrm{u}}^{++} u^{i-}, & \delta_{\mathrm{v}}^{*} u^{i-} & =0, \\
\delta_{\mathrm{v}}^{*} z^{++} & =-\lambda_{\mathrm{u}}^{++}, & \delta_{\mathrm{v}}^{*} z^{0} & =-\lambda_{\mathrm{u}}^{0} z^{0},
\end{aligned}
$$

and

$$
\delta_{\mathrm{w}}^{*} u^{i+}=-\rho_{\mathrm{u}}^{0} u^{i+}, \quad \delta_{\mathrm{w}}^{*} u^{i-}=-\rho_{\mathrm{u}}^{--} u^{i+}-\rho_{\mathrm{u}}^{0},
$$

${ }^{6}$ We denote an infinitesimal (passive) general coordinate transformation by $\delta^{*} x^{m}=-\xi^{m}$. The corresponding (active) diffeomorphism induced on a scalar field $f(x)$ is always written $\delta f(x)=\xi^{m} \partial_{m} f(x)$. This notation is opposite that employed in [2]. 


$$
\delta_{\mathrm{w}}^{*} z^{++}=\rho_{\mathrm{u}}^{++}, \quad \delta_{\mathrm{w}}^{*} z^{0}=\rho_{\mathrm{u}}^{0} z^{0}, \quad \delta_{\mathrm{w}}^{*} z^{--}=\rho_{\mathrm{u}}^{--}+2 z^{--} \rho_{\mathrm{u}}^{0} .
$$

The $\mathrm{SU}(2)_{\mathrm{v}}$ transformations with parameters $\lambda^{i j}$, which will be identified with the $\mathrm{SU}(2)_{R}$ gauge transformations of conformal supergravity in the central basis, are generated by asymmetric transformations of the complex harmonics. The diagonal isometry group generated by $\rho^{i j}=\lambda^{i j}$ corresponds to the external group of automorphisms $\mathrm{SU}(2)_{A}$ on $u^{i \pm}$, taking $\delta^{*} u^{i \pm}=\lambda^{i}{ }_{j} u^{j \pm}$ and leaving $z^{ \pm \pm}$and $z^{0}$ invariant.

We have denoted the derivatives of the complex harmonics by simple partial derivatives $\partial_{\mathrm{u}}^{ \pm \pm}$and $\partial_{\mathrm{u}}^{0}$ to emphasize that they are not covariant with respect to $\mathrm{SU}(2)_{\mathrm{v}} \times \mathrm{SU}(2)_{\mathrm{w}}$. Following [38], one can introduce covariant derivatives $\mathscr{D}^{ \pm \pm}$and $\mathscr{D}^{0}$ defined by

$$
\mathscr{D}^{ \pm \pm}:=D_{\mathrm{v}}^{ \pm \pm}+D_{\mathrm{w}}^{ \pm \pm}, \quad \mathscr{D}^{0}:=D_{\mathrm{v}}^{0}+D_{\mathrm{w}}^{0}
$$

These obey the usual algebra $\left[\mathscr{D}^{++}, \mathscr{D}^{--}\right]=\mathscr{D}^{0}$ and $\left[\mathscr{D}^{0}, \mathscr{D}^{ \pm \pm}\right]= \pm 2 \mathscr{D}^{ \pm \pm}$and act on $D_{\mathrm{v} a}$ and $D_{\mathrm{w} \bar{a}}$ as external automorphisms, e.g. $\left[\mathscr{D}^{ \pm \pm}, D_{\mathrm{v}}^{\mp \mp}\right]= \pm D_{\mathrm{v}}^{0}$ and $\left[\mathscr{D}^{0}, D_{\mathrm{v}}^{ \pm \pm}\right]= \pm 2 D_{\mathrm{v}}^{0}$. Note that the $\mathrm{SU}(2)_{\mathrm{v}}$ derivatives were denoted $Z^{ \pm \pm}$and $Z^{0}$ in [38].

In the complex harmonic coordinates, twisted biholomorphic functions are independent of $z^{++}$and $z^{--}$, while their dependence on $z^{0}$ is constrained to a single overall factor,

$$
\mathcal{F}^{(n, m)}=\left(z^{0}\right)^{n} \sum_{k=0}^{\infty} \mathcal{F}^{\left(i_{1} \cdots i_{n+m+k} j_{1} \cdots j_{k}\right)} u_{i_{1}}^{+} \cdots u_{i_{n+m+k}}^{+} u_{j_{1}}^{-} \cdots u_{j_{k}}^{-}=\left(z^{0}\right)^{n} \mathcal{F}^{(n+m)}\left(u^{ \pm}\right)
$$

where $\mathcal{F}^{(n+m)}\left(u^{ \pm}\right)$is a convergent expansion. Observe that

$$
\mathscr{D}^{++} \mathcal{F}^{(n, m)}=D_{\mathrm{w}}^{++} \mathcal{F}^{(n, m)}=\partial_{\mathrm{u}}^{++} \mathcal{F}^{(n, m)}-m z^{++} \mathcal{F}^{(n, m)}
$$

is not twisted biholomorphic unless $m=0$. Similarly, $\mathscr{D}^{--} \mathcal{F}^{(n, m)}=D_{\mathrm{v}}^{--} \mathcal{F}^{(n, m)}$ is not twisted biholomorphic unless $n$ vanishes.

\subsection{Complex harmonic integration}

In the remainder of this paper, we will primarily work with the harmonics $v^{i \pm}$ and $w^{i \pm}$, but it is enlightening to rewrite some of the previous formulae using the complex harmonics. For example, the integral $(2.10)$ becomes, using $\omega^{(-2,+2)}\left(v^{+}, w^{-}\right)=\left(z^{0}\right)^{-2} \omega\left(u^{ \pm}\right)$,

$$
S=\frac{i}{2 \pi} \int_{\mathcal{S}} \mathcal{U}^{++} \wedge \mathcal{U}^{--} \omega\left(u^{ \pm}\right)
$$

Because $\omega$ is closed, the integral is unchanged if we continuously deform $\mathcal{S}$ to $S^{2}$. This is apparent in the above form as the integrand is manifestly independent of the coordinates $z^{++}, z^{--}$and $z^{0}$, so we may certainly choose $z^{++}=z^{--}=0$ and $z^{0}=1$. This recovers the usual notion of harmonic integration.

If instead we have the more general two-form (2.11), it is convenient to rewrite

$$
\omega^{(-2,2)}\left(v^{+}, w^{-}\right)=\frac{1}{\left(z^{0}\right)^{2}} \omega\left(u^{ \pm}, z^{--}\right), \quad \omega^{(2,2)}\left(v^{+}, w^{-}\right)=\left(z^{0}\right)^{2} \omega^{+4}\left(u^{ \pm}, z^{--}\right) .
$$


The components are each independent of $z^{++}$and are required to obey

$$
\frac{\partial}{\partial z^{--}} \omega\left(u^{ \pm}, z^{--}\right)=\left(\partial_{\mathrm{u}}^{--}+2 z^{--}+\left(z^{--}\right)^{2} \frac{\partial}{\partial z^{--}}\right) \omega^{+4}\left(u^{ \pm}, z^{--}\right) .
$$

The action principle is now a bit more complicated for a general surface $\mathcal{S}$,

$$
S=\frac{i}{2 \pi} \int_{\mathcal{S}} \mathcal{U}^{++} \wedge \mathcal{U}^{--} \omega+\frac{i}{2 \pi} \int_{\mathcal{S}} \mathcal{U}^{--} \wedge\left(2 z^{--} \mathcal{U}^{0}+\left(z^{--}\right)^{2} \mathcal{U}^{++}+\mathrm{d} z^{--}\right) \omega^{+4}
$$

For these two-forms, it is more convenient to use the original expressions with $v_{i}^{ \pm}$and $w_{i}^{ \pm}$.

One may introduce analogues of Stokes' theorem just as before, but as we will mainly be working with the original harmonics $v^{ \pm}$and $w^{ \pm}$, reformulating (2.14) for complex harmonics will not be necessary. However, it is useful to note that when the integrands are twisted biholomorphic,

$$
\int_{\mathcal{S}} \mathcal{U}^{++} \wedge \mathcal{U}^{--} \partial_{\mathrm{u}}^{--} \Lambda^{++}\left(u^{ \pm}\right)=0, \quad \int_{\mathcal{S}} \mathcal{U}^{++} \wedge \mathcal{U}^{--} \partial_{\mathrm{u}}^{++} \Lambda^{--}\left(u^{ \pm}\right)=0
$$

Using these identities, we can prove a number of results that establish that complex harmonic integration works exactly as real harmonic integration. First, one can show that

$$
\frac{i}{2 \pi} \int_{\mathcal{S}} \mathcal{U}^{++} \wedge \mathcal{U}^{--} u_{\left(i_{1}\right.}^{+} \cdots u_{i_{\ell}}^{+} u_{j_{1}}^{-} \cdots u_{\left.j_{\ell}\right)}^{-}=0 \quad \text { for } \quad \ell \geq 1
$$

for any closed surface $\mathcal{S}$. This follows by choosing $\Lambda^{--}=u_{\left(i_{1}\right.}^{+} \cdots u_{i_{\ell-1}}^{+} u_{i_{\ell}}^{-} u_{j_{1}}^{-} \cdots u_{\left.j_{\ell}\right)}^{-}$ in (2.36). Similar identities with unequal numbers of symmetrized positive and negative harmonics can be established. We would like to also impose the normalization condition $\frac{i}{2 \pi} \int_{\mathcal{S}} \mathcal{U}^{++} \wedge \mathcal{U}^{--}=1$. This obviously holds when $\mathcal{S}=S^{2}$ - we chose the overall normalization of the integral to ensure this - and holds more generally because the integrand is closed. It follows that

$$
\frac{i}{2 \pi} \int_{\mathcal{S}} \mathcal{U}^{++} \wedge \mathcal{U}^{--} \equiv \frac{i}{2 \pi} \int_{\mathcal{S}} \mathcal{V}^{++} \wedge \mathcal{W}^{--}\left(z^{0}\right)^{-2}=\frac{i}{2 \pi} \int_{\mathcal{S}} \mathcal{V}^{++} \wedge \mathcal{V}^{--}=1
$$

These results will prove crucial when performing component reductions in superspace.

\section{Complex harmonic superspace on $\mathcal{M}^{4 \mid 8} \times \mathrm{SU}(2)_{\mathrm{v}} \times \mathrm{SU}(2)_{\mathrm{w}}$}

Now we are prepared to introduce the first main result of this paper: the construction of complex harmonic superspace on the supermanifold $\mathcal{M}^{4 \mid 8} \times \mathrm{SU}(2)_{\mathrm{v}} \times \mathrm{SU}(2)_{\mathrm{w}}$. This approach is based on the projective superspace $\mathcal{M}^{4 \mid 8} \times \mathrm{SU}(2)_{\mathrm{v}}$ elaborated upon in [10], with the underlying structure of supergravity on $\mathcal{M}^{4 \mid 8}$ described by $\mathcal{N}=2$ conformal superspace [45].

Conformal superspace is a recent approach in the superspace literature that gauges the entire superconformal group including dilatations, special conformal transformations, and $S$-supersymmetry; it is precisely the superspace version of the superconformal tensor calculus [46]. In contrast to other superspace formulations gauging at most the Lorentz and $R$-symmetry groups, conformal superspace proves to be quite economical and simple to work with, as seen in the rather simple algebra of covariant spinor derivatives. 


\subsection{Construction in the central basis}

Let us begin by recalling the salient details of [10]. That superspace, which we can identify as $\mathcal{M}^{7 \mid 8}=\mathcal{M}^{4 \mid 8} \times \mathrm{SU}(2)_{\mathrm{v}}$, involves local coordinates $z^{\underline{M}}=\left(z^{M}, y^{\underline{\underline{m}}}\right)$ and a vielbein $E_{\underline{M}^{\underline{A}}}$ given in block form as

$$
E_{\underline{M}}^{\underline{A}}=\left(\begin{array}{ll}
E_{M}^{A} & E_{M}^{\mathrm{v} \underline{a}} \\
E_{\underline{m}}^{A} & E_{\underline{\underline{m}}}^{\mathrm{v} \underline{a}}
\end{array}\right),
$$

where the tangent space index $A=(a, \underline{\alpha} \pm)=(a, \alpha \pm, \dot{\alpha} \pm)$ is associated with $\mathcal{M}^{4 \mid 8}$ and $\underline{a}=$ $( \pm \pm, 0)$ is associated with $\mathrm{SU}(2)_{\mathrm{v}}$. The covariant derivatives $\nabla_{\underline{A}}=\left(\nabla_{a}, \nabla_{\underline{\alpha} \pm}, \nabla_{\mathrm{v} \pm \pm}, \nabla_{\mathrm{v} 0}\right)$ are defined by the relation

$$
\partial_{\underline{M}}=E_{\underline{M}}^{\underline{A}} \nabla_{\underline{A}}+\frac{1}{2} \Omega_{\underline{M}}^{a b} M_{b a}+A_{\underline{M}} \mathbb{A}+B_{\underline{M}} \mathbb{D}+F_{\underline{M}}^{A} K_{A},
$$

involving the Lorentz generator $M_{a b}$, the dilatation generator $\mathbb{D}$, the $\mathrm{U}(1)_{R}$ generator $\mathbb{A}$, and the special (super)conformal generators $K_{A}=\left(K_{a}, S_{\underline{\alpha} \pm}\right)$. The Lorentz, dilatation, and $\mathrm{U}(1)_{R}$ generators are normalized as

$$
\begin{aligned}
{\left[M_{a b}, \nabla_{\gamma}^{ \pm}\right] } & =\left(\sigma_{a b}\right)_{\gamma}{ }^{\beta} \nabla_{\beta}^{ \pm}, & {\left[M_{a b}, \bar{\nabla}^{\dot{\gamma} \pm}\right] } & =\left(\bar{\sigma}_{a b}\right)_{\dot{\beta}}^{\dot{\gamma}} \bar{\nabla}^{\dot{\beta} \pm}, & {\left[M_{a b}, \nabla_{c}\right] } & =\eta_{b c} \nabla_{a}-\eta_{a c} \nabla_{b}, \\
{\left[\mathbb{D}, \nabla_{\alpha}^{ \pm}\right] } & =\frac{1}{2} \nabla_{\alpha}^{ \pm}, & {\left[\mathbb{D}, \bar{\nabla}^{\dot{\alpha} \pm}\right] } & =\frac{1}{2} \bar{\nabla}^{\dot{\alpha} \pm}, & & {\left[\mathbb{D}, \nabla_{a}\right]=\nabla_{a}, } \\
{\left[\mathbb{A}, \nabla_{\alpha}^{ \pm}\right] } & =-i \nabla_{\alpha}^{ \pm}, & {\left[\mathbb{A}, \bar{\nabla}^{\dot{\alpha} \pm}\right] } & =+i \bar{\nabla}^{\dot{\alpha} \pm} . & &
\end{aligned}
$$

We use the following prescription for raising the \pm tangent space indices,

$$
\nabla_{\underline{\alpha} \mp}= \pm \nabla_{\underline{\alpha}}^{ \pm}, \quad \nabla_{\mathrm{v} \mp \mp}= \pm \nabla_{\mathrm{v}}^{ \pm \pm}, \quad S_{\underline{\alpha} \mp}=\mp S_{\underline{\alpha}}^{ \pm}, \quad \nabla_{\mathrm{v} 0}=\nabla_{\mathrm{v}}^{0},
$$

so that they corresponded to the $\nabla_{\mathrm{v}}^{0}$ charge of the operators. The algebra of the special superconformal generators with the spinor derivatives generates the $\mathrm{SU}(2)_{\mathrm{v}}$ derivatives,

$$
\begin{aligned}
\left\{S_{\beta}^{ \pm}, \nabla_{\alpha}^{ \pm}\right\} & = \pm 4 \epsilon_{\beta \alpha} \nabla_{\mathrm{v}}^{ \pm \pm}, \quad\left\{\bar{S}^{\dot{\beta} \pm}, \bar{\nabla}^{\dot{\alpha} \pm}\right\}=\mp 4 \epsilon^{\dot{\beta} \dot{\alpha}} \nabla_{\mathrm{v}}^{ \pm \pm}, \\
\left\{S_{\beta}^{\mp}, \nabla_{\alpha}^{ \pm}\right\} & = \pm\left(2 \epsilon_{\beta \alpha} \mathbb{D}-2 M_{\beta \alpha}-i \epsilon_{\beta \alpha} \mathbb{A}\right)-2 \epsilon_{\beta \alpha} \nabla_{\mathrm{v}}^{0}, \\
\left\{\bar{S}^{\dot{\beta} \mp}, \bar{\nabla}^{\dot{\alpha} \pm}\right\} & =\mp\left(2 \epsilon^{\dot{\beta} \dot{\alpha}} \mathbb{D}-2 M^{\dot{\beta} \dot{\alpha}}+i \epsilon^{\dot{\beta} \dot{\alpha}} \mathbb{A}\right)+2 \epsilon^{\dot{\beta} \dot{\alpha}} \nabla_{\mathrm{v}}^{0} .
\end{aligned}
$$

This identifies $\mathrm{SU}(2)_{\mathrm{v}}$ with the superconformal group $\mathrm{SU}(2)_{R}$. The remaining relations between these generators and their action on the covariant derivatives can be found in [10].

In the central basis (or central gauge), the vielbein decomposes as $E^{A}=\mathrm{d} z^{M} E_{M}{ }^{A}$ and $E^{\mathrm{v}} \underline{\underline{a}} \equiv \mathcal{V}^{\underline{a}}=\mathrm{d} y^{\underline{m}} \underline{V}_{\underline{m}}^{\underline{a}}+\mathrm{d} z^{M} \mathcal{V}_{M^{\underline{a}}}$, or in block form

$$
E_{\underline{M}^{\underline{A}}}=\left(\begin{array}{cc}
E_{M} & \mathcal{V}_{M^{\underline{a}}} \\
0 & \mathcal{V}_{\underline{m}^{\underline{a}}}^{\underline{a}}
\end{array}\right) .
$$

The components of $E^{A}$ correspond to the vielbein on $\mathcal{M}^{4 \mid 8}$, while the vielbein $E^{\mathrm{v} a}$, which we rename to $\mathcal{V}^{\underline{a}}$ in the central basis for convenience, decomposes into the vielbein $\underline{\mathcal{V}}_{\underline{m}} \underline{a}$ on 
$\mathrm{SU}(2)_{\mathrm{v}}$ and the $\mathrm{SU}(2)_{R}$ connection $\mathcal{V}_{M^{\underline{a}}}$ on $\mathcal{M}^{4 \mid 8}$. The covariant derivatives may then be grouped into the $\mathcal{M}^{4 \mid 8}$ covariant derivative

$$
\nabla_{A}=E_{A}^{M}\left(\partial_{M}-\mathcal{V}_{M}^{\underline{a}} D_{\underline{a}}-\frac{1}{2} \Omega_{M}^{b c} M_{c b}-A_{M} \mathbb{A}-B_{M} \mathbb{D}-F_{M}^{B} K_{B}\right)
$$

with $E_{A}{ }^{M}=\left(E_{M}^{A}\right)^{-1}$ and the $\mathrm{SU}(2)_{\mathrm{v}}$ covariant derivative

$$
\nabla_{\underline{a}} \equiv D_{\underline{a}}=\mathcal{V}_{\underline{a}}^{\underline{\underline{m}}} \partial_{\underline{m}}, \quad D_{\mathrm{v}++} \equiv-D_{\mathrm{v}}^{--}, \quad D_{\mathrm{v}--} \equiv D_{\mathrm{v}}^{++}, \quad D_{\mathrm{v} 0} \equiv D_{\mathrm{v}}^{0},
$$

with $\mathcal{V}_{\underline{a}}^{\underline{m}}=\left(\mathcal{V}_{\underline{m}}{ }^{\underline{a}}\right)^{-1}$. The constraints chosen on the curvatures imply that

$$
\nabla_{\underline{\alpha}}^{ \pm}=v_{i}^{ \pm} \nabla_{\underline{\alpha}}^{i}
$$

with the connections in the covariant derivatives $\nabla_{\underline{\alpha}}^{i}$ and $\nabla_{a}$ essentially independent of the harmonics $v^{i \pm}$.

Now let us extend this curved superspace to complex harmonic superspace. Beginning in the central basis, we attach the completely decoupled space $\mathrm{SU}(2)_{\mathrm{w}}$ with local coordinates $y^{\bar{m}}$ and vielbein $\mathcal{W}_{\bar{m}}{ }^{\bar{a}}$, obeying $\partial_{\bar{m}}=\mathcal{W}_{\bar{m}}{ }^{\bar{a}} D_{\bar{a}}$, for covariant $\mathrm{SU}(2)_{\mathrm{w}}$ derivatives $D_{\mathrm{w} \bar{a}}=$ $\left(D_{\mathrm{w}++}, D_{\mathrm{w}--}, D_{\mathrm{w} 0}\right)=\left(-D_{\mathrm{w}}^{--}, D_{\mathrm{w}}^{++}, D_{\mathrm{w}}^{0}\right)$. Denoting the full set of coordinates by $z^{\mathcal{M}}$, the full vielbein $E_{\mathcal{M}^{\mathcal{A}}}$ is in block form

$$
E_{\mathcal{M}^{\mathcal{A}}}=\left(\begin{array}{ccc}
E_{M^{A}} \mathcal{V}_{M^{\underline{a}}} & 0 \\
0 & \mathcal{V}_{\underline{\underline{m}}}^{\underline{a}} & 0 \\
0 & 0 & \mathcal{W}_{\bar{m}}^{\bar{a}}
\end{array}\right) .
$$

The other connections are even simpler,

$$
\Omega_{\mathcal{M}}^{a b}=\left(\Omega_{M}^{a b}, 0,0\right), \quad A_{\mathcal{M}}=\left(A_{M}, 0,0\right), \quad \text { etc. }
$$

and the covariant derivative $\nabla_{\mathcal{A}}=\left(\nabla_{A}, D_{\mathrm{v} \underline{\mathrm{a}}}, D_{\mathrm{w} \bar{a}}\right)$ is given by

$$
\partial_{\mathcal{M}}=E_{\mathcal{M}}{ }^{\mathcal{A}} \nabla_{\mathcal{A}}+\frac{1}{2} \Omega_{\mathcal{M}}{ }^{a b} M_{b a}+A_{\mathcal{M}} \mathbb{A}+B_{\mathcal{M}} \mathbb{D}+F_{\mathcal{M}}{ }^{A} K_{A}
$$

In the central basis, there is a clear distinction between $\mathrm{SU}(2)_{\mathrm{v}}$ and $\mathrm{SU}(2)_{\mathrm{w}}$. The first is identified with the $\mathrm{SU}(2) R$-symmetry group, while the second remains decoupled. In particular, one finds for $\nabla_{A}=\left(\nabla_{a}, \nabla_{\underline{\alpha}}\right)$ the same expression (3.7) in the central basis. The covariant derivatives $\nabla_{\mathrm{v} \underline{a}}$ and $\nabla_{\mathrm{w} \bar{a}}$ retain their flat forms, $D_{\mathrm{v} \underline{a}}=\left(D_{\mathrm{v} \pm \pm}, D_{\mathrm{v} 0}\right)$ and $D_{\mathrm{w} \bar{a}}=\left(D_{\mathrm{w} \pm \pm}, D_{\mathrm{w} 0}\right)$.

Of course, there is no barrier to going to a basis (or gauge) where the vielbein and connections take a more general form. We retain the same algebra of covariant derivatives given in [10], and append the $\mathrm{SU}(2)_{\mathrm{w}}$ covariant derivatives $\nabla_{\mathrm{w} \bar{a}}=\left(\nabla_{\mathrm{w}++}, \nabla_{\mathrm{w}--}, \nabla_{\mathrm{w} 0}\right)=$ $\left(-\nabla_{\mathrm{w}}^{--}, \nabla_{\mathrm{w}}^{++}, \nabla_{\mathrm{w}}^{0}\right)$, which commute with all the other generators and covariant derivatives. The resulting algebra of covariant spinor derivatives in any gauge is

$$
\left\{\nabla_{\underline{\alpha}}^{ \pm}, \nabla_{\underline{\beta}}^{ \pm}\right\}=0
$$




$$
\left\{\nabla_{\alpha}^{ \pm}, \bar{\nabla}_{\dot{\beta}}^{\mp}\right\}=\mp 2 i \nabla_{\alpha \dot{\beta}}, \quad\left\{\nabla_{\alpha}^{ \pm}, \nabla_{\beta}^{\mp}\right\}= \pm 2 \epsilon_{\alpha \beta} \overline{\mathcal{W}}, \quad\left\{\bar{\nabla}^{\dot{\alpha} \pm}, \bar{\nabla}^{\dot{\beta} \mp}\right\}= \pm 2 \epsilon^{\dot{\alpha} \dot{\beta}} \mathcal{W}
$$

The first equation implies the existence of covariantly analytic superfields. The operator $\mathcal{W}$ appearing in the latter equations is constructed from a single complex superfield $W_{\alpha \beta}$,

$$
\begin{aligned}
& \mathcal{W}=\frac{1}{2} W^{\alpha \beta} M_{\beta \alpha}+\frac{1}{4} \nabla^{\beta+} W_{\beta}{ }^{\alpha} S_{\alpha}^{-}-\frac{1}{4} \nabla^{\beta-} W_{\beta}{ }^{\alpha} S_{\alpha}^{+}+\frac{1}{4} \nabla^{\dot{\alpha} \beta} W_{\beta}{ }^{\alpha} K_{\alpha \dot{\alpha}}, \\
& \overline{\mathcal{W}}=\frac{1}{2} \bar{W}_{\dot{\alpha} \dot{\beta}} M^{\dot{\beta} \dot{\alpha}}+\frac{1}{4} \bar{\nabla}_{\dot{\beta}}^{-} \bar{W}^{\dot{\beta}} \bar{S}^{\dot{\alpha}+}-\frac{1}{4} \bar{\nabla}_{\dot{\beta}}^{+} \bar{W}^{\dot{\beta}} \dot{\alpha} \bar{S}^{\dot{\alpha}-}+\frac{1}{4} \nabla_{\alpha \dot{\beta}} \bar{W}^{\dot{\beta}} \dot{\alpha} K^{\dot{\alpha} \alpha} .
\end{aligned}
$$

$W_{\alpha \beta}$ is covariantly independent of the harmonics and is the single curvature superfield of conformal superspace [45]. The remaining curvatures may be compactly written

$$
\begin{aligned}
{\left[\nabla_{\beta}^{ \pm}, \nabla_{\alpha \dot{\alpha}}\right] } & =-2 \epsilon_{\beta \alpha} \overline{\mathcal{W}}_{\dot{\alpha}}^{ \pm}, \quad\left[\bar{\nabla}_{\dot{\beta}}^{ \pm}, \nabla_{\alpha \dot{\alpha}}\right]=-2 \epsilon_{\dot{\beta} \dot{\alpha}} \mathcal{W}_{\alpha}^{ \pm}, \\
{\left[\nabla_{\beta \dot{\beta}}, \nabla_{\alpha \dot{\alpha}}\right] } & =-\mathcal{F}_{\beta \dot{\beta} \alpha \dot{\alpha}}=-2 \epsilon_{\dot{\beta} \dot{\alpha}} \mathcal{F}_{\beta \alpha}+2 \epsilon_{\beta \alpha} \mathcal{F}_{\dot{\beta} \dot{\alpha}} .
\end{aligned}
$$

The spinor operators $\mathcal{W}_{\underline{\alpha}}^{ \pm}$and anti-selfdual and selfdual components of $\mathcal{F}_{b a}$ are given by

$$
\mathcal{W}_{\underline{\alpha}}^{ \pm}=-\frac{i}{2}\left[\nabla_{\underline{\alpha}}^{ \pm}, \mathcal{W}\right], \quad \mathcal{F}_{\beta \alpha}=\frac{1}{4}\left\{\nabla_{(\beta}^{+},\left[\nabla_{\alpha)}^{-}, \mathcal{W}\right]\right\}, \quad \mathcal{F}_{\dot{\beta} \dot{\alpha}}=\frac{1}{4}\left\{\bar{\nabla}_{(\dot{\beta}}^{+},\left[\bar{\nabla}_{\dot{\alpha})}^{-}, \overline{\mathcal{W}}\right]\right\} .
$$

Explicit expressions for these can be found in [10]. The simplicity of these relations is one of the main advantages of conformal superspace.

In a general gauge, an arbitrary covariant diffeomorphism and gauge transformation may be written

$$
\delta=\xi^{\mathcal{A}} \nabla_{\mathcal{A}}+\frac{1}{2} \lambda^{a b} M_{b a}+\Lambda_{\mathrm{D}} \mathbb{D}+\Lambda_{\mathrm{A}} \mathbb{A}+\eta^{\underline{\alpha}}+S_{\underline{\alpha}}^{-}-\eta^{\underline{\alpha}}-S_{\underline{\alpha}}^{+}+\epsilon^{a} K_{a}
$$

in terms of arbitrary parameters $\xi^{\mathcal{A}}, \lambda^{a b}, \Lambda_{\mathrm{A}}, \Lambda_{\mathrm{D}}, \epsilon^{a}$ and $\eta^{\underline{\alpha}}$. We remind that

$$
\begin{aligned}
\xi^{\mathcal{A}} \nabla_{\mathcal{A}}= & \xi^{a} \nabla_{a}+\xi^{\underline{\underline{\alpha}}-} \nabla_{\underline{\alpha}}^{+}-\xi^{\underline{\alpha}+} \nabla_{\underline{\alpha}}^{-}+\xi_{\mathrm{v}}^{--} \nabla_{\mathrm{v}}^{++}+\xi_{\mathrm{v}}^{0} \nabla_{\mathrm{v}}^{0}-\xi_{\mathrm{v}}^{++} \nabla_{\mathrm{v}}^{--} \\
& +\xi_{\mathrm{w}}^{--} \nabla_{\mathrm{w}}^{++}+\xi_{\mathrm{w}}^{0} \nabla_{\mathrm{w}}^{0}-\xi_{\mathrm{w}}^{++} \nabla_{\mathrm{w}}^{--} .
\end{aligned}
$$

The charges on each parameter refer to their $\mathrm{SU}(2)_{\mathrm{v}}$ charge, except for the local $\mathrm{SU}(2)_{\mathrm{w}}$ parameters $\xi_{\mathrm{w}}^{ \pm \pm}$. In practice, one should restrict to gauges connected to the central basis by complex harmonic gauge transformations, that is, gauge transformations that are at most twisted biholomorphic on $\mathrm{SU}(2)_{\mathrm{v}} \times \mathrm{SU}(2)_{\mathrm{w}}$. This means that the vielbein and connections will generally be constrained so that $\nabla_{\mathrm{w}}^{++}$and $\nabla_{\mathrm{v}}^{--}$acquire additional connections while the other covariant harmonic derivatives remain relatively simple.

In the central basis, the harmonic dependence on the parameters is restricted to maintain the block form (3.10),

$$
\begin{aligned}
\xi^{\underline{\alpha}} \pm & =v_{i}^{ \pm} \xi^{\underline{\alpha} i}, & \eta^{\underline{\alpha}} & =v_{i}^{ \pm} \eta^{\underline{\alpha} i} \\
\xi_{\mathrm{v}}^{ \pm \pm} & \equiv \lambda_{\mathrm{v}}^{ \pm \pm}=v_{i}^{ \pm} v_{j}^{ \pm} \lambda^{i j}, & \xi_{\mathrm{v}}^{0} & \equiv \lambda_{\mathrm{v}}^{0}=v_{i}^{+} v_{j}^{-} \lambda^{i j}, \\
\xi_{\mathrm{w}}^{ \pm \pm} & \equiv \rho_{\mathrm{w}}^{ \pm \pm}=w_{i}^{ \pm} w_{j}^{ \pm} \rho^{i j}, & \xi_{\mathrm{w}}^{0} & \equiv \rho_{\mathrm{w}}^{0}=w_{i}^{+} w_{j}^{-} \rho^{i j}
\end{aligned}
$$


with the other parameters harmonic-independent. Moreover, the absence of $\mathrm{SU}(2)_{\mathrm{w}}$ connections means that $\rho^{i j}$ are always constants in the central basis. In fact, we may refrain from ever performing $\mathrm{SU}(2)_{\mathrm{w}}$ diffeomorphisms.

This is perhaps a good place to emphasize again that the derivatives $\nabla_{\alpha}^{ \pm}$are given in the central basis by (3.9) and not, as one might otherwise expect, by $u_{i}^{ \pm} \nabla_{\underline{\alpha}}{ }^{\bar{i}}$. That is, the algebra they obey with the $\mathrm{SU}(2)_{\mathrm{v}}$ and $\mathrm{SU}(2)_{\mathrm{w}}$ derivatives can be written

$$
\begin{array}{llll}
{\left[D_{\mathrm{v}}^{ \pm \pm}, \nabla_{\underline{\alpha}}^{ \pm}\right]=0,} & {\left[D_{\mathrm{v}}^{ \pm \pm}, \nabla_{\underline{\alpha}}^{\mp}\right]=\nabla_{\underline{\alpha}}^{ \pm},} & & {\left[D_{\mathrm{v}}^{0}, \nabla_{\underline{\alpha}}^{ \pm}\right]= \pm \nabla_{\underline{\alpha}}^{ \pm},} \\
{\left[D_{\mathrm{w}}^{ \pm \pm}, \nabla_{\underline{\alpha}}^{ \pm}\right]=0,} & {\left[D_{\mathrm{w}}^{ \pm \pm}, \nabla_{\underline{\alpha}}^{\mp}\right]=0,} & {\left[D_{\mathrm{w}}^{0}, \nabla_{\underline{\alpha}}^{ \pm}\right]=0 .}
\end{array}
$$

The closest analogues of the conventional harmonic derivative relations are

$$
\left[\mathscr{D}^{ \pm \pm}, \nabla_{\underline{\alpha}}^{ \pm}\right]=0, \quad\left[\mathscr{D}^{ \pm \pm}, \nabla_{\underline{\alpha}}^{\mp}\right]=\nabla_{\underline{\alpha}}^{ \pm}, \quad\left[\mathscr{D}^{0}, \nabla_{\underline{\alpha}}^{ \pm}\right]= \pm \nabla_{\underline{\alpha}}^{ \pm},
$$

using the derivatives $\mathscr{D}:=D_{\mathrm{v}}+D_{\mathrm{w}}$ defined in section 2.3. These commutators hold in any gauge, replacing $D_{\mathrm{v}} \rightarrow \nabla_{\mathrm{v}}$ and $D_{\mathrm{w}} \rightarrow \nabla_{\mathrm{w}}$.

\subsection{Covariant primary analytic superfields}

Because the covariant spinor derivatives obey the conditions (3.13), the superspace admits analytic superfields $\Psi$ obeying $\nabla_{\underline{\alpha}}^{+} \Psi=0$. We are interested only in superfields that are also primary, $S_{\underline{\alpha}}^{ \pm} \Psi=K_{a} \Psi=0$. Consistency with the operator algebra implies that $\Psi$ is a Lorentz scalar, invariant under $\mathrm{U}(1)_{R}$, and obeys ${ }^{7}$

$$
\nabla_{\mathrm{v}}^{0} \Psi=\mathbb{D} \Psi, \quad \nabla_{\mathrm{v}}^{++} \Psi=0
$$

In other words, $\Psi$ must have a $\mathrm{U}(1)_{\mathrm{v}}$ charge equal to its conformal dimension - for definiteness, let us denote both quantities by $n$ - and $\Psi$ must be holomorphic on an open domain of $\mathrm{SU}(2)_{\mathrm{v}}$. We may further choose this open domain to be the vicinity of the diagonal $\mathrm{SU}(2)$ of $\mathrm{SU}(2)_{\mathrm{v}} \times \mathrm{SU}(2)_{\mathrm{w}}$ and restrict $\Psi$ to be a twisted biholomorphic scalar $\mathcal{F}^{(n, m)}$ with charges $(n, m)$ under $\mathrm{U}(1)_{\mathrm{v}} \times \mathrm{U}(1)_{\mathrm{w}}$. A general conformal supergravity transformation of such a superfield is

$$
\delta \mathcal{F}^{(n, m)}=\xi^{A} \nabla_{A} \mathcal{F}^{(n, m)}+n\left(\Lambda_{\mathrm{D}}+\lambda_{\mathrm{v}}^{0}\right) \mathcal{F}^{(n, m)}-\lambda_{\mathrm{v}}^{++} D_{\mathrm{v}}^{--} \mathcal{F}^{(n, m)}
$$

when written in the central basis. In terms of the complex harmonic coordinates, one finds

$$
\delta \mathcal{F}^{(n, m)}=\xi^{A} \nabla_{A} \mathcal{F}^{(n, m)}+n\left(\Lambda_{\mathrm{D}}+\lambda_{\mathrm{u}}^{0}\right) \mathcal{F}^{(n, m)}-\lambda_{\mathrm{u}}^{++} \partial_{\mathrm{u}}^{--} \mathcal{F}^{(n, m)} .
$$

Below we will summarize the various types of multiplets commonly encountered in harmonic superspace (see e.g. [2] for further details and references) and discuss their twisted biholomorphic description in the central basis.

\footnotetext{
${ }^{7}$ These conditions were discussed in chapter 9 of the monograph [2]. They are also the conditions required for covariant projective multiplets [24].
} 
$\mathcal{O}(\boldsymbol{n})$ multiplets. In flat harmonic superspace, one can introduce complex $\mathcal{O}(n)$ multiplets $[47,48]$ that obey $D^{++} \mathcal{H}^{(n)}=D_{\underline{\alpha}}^{+} \mathcal{H}^{(n)}=0$. The generalization to curved harmonic superspace is straightforward: we need twisted biholomorphic analytic superfields $\mathcal{H}^{(n, m)}$ obeying the additional restriction $D_{\mathrm{w}}^{++} \mathcal{H}^{(n, m)}=0$. As a consequence of the twisted biholomorphy, one finds the integrability condition $\left[D_{\mathrm{w}}^{++}, D_{\mathrm{w}}^{--}\right] \mathcal{H}^{(n, m)}=m \mathcal{H}^{(n, m)}=0$, so we are restricted to superfields $\mathcal{H}^{(n, 0)}$ with

$$
\mathcal{H}^{(n, 0)}=\mathcal{H}^{j_{1} \cdots j_{n}} v_{j_{1}}^{+} \cdots v_{j_{n}}^{+}, \quad \nabla_{\underline{\alpha}}^{(i} \mathcal{H}^{\left.j_{1} \cdots j_{n}\right)}=0 .
$$

As a consequence of $(3.23), \mathcal{H}^{(n, 0)}$ must have weight $n$ under dilatations. If $n$ is an even integer, it is possible to impose a reality condition. The most familiar such multiplet is the $\mathcal{O}(2)$ multiplet, or tensor multiplet, $\mathcal{G}^{++}=\mathcal{G}^{i j} v_{i}^{+} v_{j}^{+}$, which plays a major role as a compensator in one of the off-shell formulations of $\mathcal{N}=2$ Poincaré supergravity [49]. It possesses the same form in either complex harmonic or projective superspace, and the same holds for the general complex $\mathcal{O}(n)$ multiplets.

Relaxed hypermultiplets. We next consider the general class of so-called relaxed hypermultiplets. In flat harmonic superspace, these are given by analytic superfields $\mathcal{R}^{+q}$ obeying $\left(D^{++}\right)^{p} \mathcal{R}^{+q}=0$ for some set of integers $p$ and $q$ (see [2] for a discussion and further references). Their generalization in curved harmonic superspace involves twisted biholomorphic superfields $\mathcal{R}^{(n, m)}$ with $n+m=q$ and the constraint $\left(D_{\mathrm{w}}^{++}\right)^{p} \mathcal{R}^{(n, m)}=0$. As a consequence of the twisted biholomorphic condition, one finds $m=1-p$.

To understand this condition, it helps to specialize to the case where $n+m=2$ and $p=2$. Here one finds a superfield $\mathcal{R}^{(3,-1)}$ with

$$
\mathcal{R}^{(3,-1)}=\left(z^{0}\right)^{3}\left(\mathcal{R}^{(i j)} u_{i}^{+} u_{j}^{+}+\mathcal{R}^{(i j k \mathrm{a})} u_{i}^{+} u_{j}^{+} u_{k}^{+} u_{\mathrm{a}}^{-}\right) .
$$

We have denoted the index of the negative harmonic with a Roman index to distinguish it from the others. This is because the above expression can be rewritten

$$
\mathcal{R}^{(3,-1)}=\mathcal{R}^{i j k \mathrm{a}} v_{i}^{+} v_{j}^{+} v_{k}^{+} w_{\mathrm{a}}^{-},
$$

where $\mathcal{R}^{i j k \mathrm{a}}=\mathcal{R}^{(i j k) \mathrm{a}}$ is symmetric in its first three indices only. The expression (3.27) is recovered by decomposing $\mathcal{R}^{i j k \mathrm{a}}=-\mathcal{R}^{(i j} \epsilon^{k) \mathrm{a}}+\mathcal{R}^{(i j k \mathrm{a})}$. The form (3.28) is advantageous for several reasons: the constraint is manifestly satisfied for $p=2$, the analyticity condition amounts to $\nabla_{\underline{\alpha}}^{(l} \mathcal{R}^{i j k) \mathrm{a}}=0$, and the transformation (3.24) leads to

$$
\delta \mathcal{R}^{i j k \mathrm{a}}=\xi^{A} \nabla_{A} \mathcal{R}^{i j k \mathrm{a}}+3 \Lambda_{\mathrm{D}} \mathcal{R}^{i j k \mathrm{a}}+3 \lambda^{(i}{ }_{l} \mathcal{R}^{j k) l \mathrm{a}} .
$$

This is consistent with the simple interpretation that $\mathcal{R}^{i j k \text { a }}$ possesses three $\mathrm{SU}(2)_{R}$ indices associated with the isometric action on $\mathrm{SU}(2)_{\mathrm{v}}$, and an additional external $\mathrm{SU}(2)$ index associated with $\mathrm{SU}(2)_{\mathrm{w}}$. The superfield $\mathcal{R}^{i j k \mathrm{a}}$ is just a globally defined $\mathcal{O}(3)$ superfield $\mathcal{R}^{(3) a}$ in projective superspace with an extra inert index. It is naturally embedded into complex harmonic superfield by writing $\mathcal{R}^{(3,-1)} \equiv \mathcal{R}^{(3) \mathrm{a}} w_{\mathrm{a}}^{-}$.

In like fashion, the general relaxed hypermultiplet $\mathcal{R}^{(n, 1-p)}$ obeying $\left(D_{\mathrm{w}}^{++}\right)^{p} \mathcal{R}^{(n, 1-p)}=$ 0 , is associated with a harmonic-independent superfield $\mathcal{R}^{i_{1} \cdots i_{n} a_{1} \cdots a_{p-1}}$. This can be interpreted as a projective superspace $\mathcal{O}(n)$ multiplet $\mathcal{R}^{(n) \mathrm{a}_{1} \cdots \mathrm{a}_{p-1}}$ with $p-1$ symmetric external $\mathrm{SU}(2)$ indices. 
The nonlinear multiplet. Our third example is the nonlinear multiplet. Within real harmonic superspace, it is given by an analytic superfield $\mathrm{N}^{++}$obeying the constraint $D^{++} N^{++}=-\left(N^{++}\right)^{2}$. Because the complex harmonic version of this analytic superfield must be weight zero under dilatations, it must have vanishing $D_{\mathrm{v}}^{0}$ charge, and so it should be given by an analytic multiplet $N^{(0,2)}$. As usual, analyticity implies that $D_{\mathrm{v}}^{++} N^{(0,2)}=0$, so the constraint must be given by $D_{\mathrm{w}}^{++}$. In fact, it turns out two constraints are needed,

$$
D_{\mathrm{w}}^{++} N^{(0,2)}=-\left(N^{(0,2)}\right)^{2}, \quad D_{\mathrm{w}}^{--} N^{(0,2)}=1 .
$$

The second implies that $N^{(0,2)}$ is not twisted biholomorphic, but instead possesses some dependence on $w^{i+}$. This leads the conformal supergravity transformation $\delta N^{(0,2)}=$ $\xi^{A} \nabla_{A} N^{(0,2)}-\lambda_{\mathrm{v}}^{++} D_{\mathrm{v}}^{--} N^{(0,2)}$ to take an unusual form when written in terms of complex harmonic coordinates:

$$
\delta N^{(0,2)}=\xi^{A} \nabla_{A} N^{(0,2)}-\lambda_{\mathrm{u}}^{++} \partial_{\mathrm{u}}^{--} N^{(0,2)}-\lambda_{\mathrm{u}}^{++} .
$$

The inhomogeneous term appears also in the conventional harmonic superspace description of this multiplet: there it arises as consistency condition for the constraint.

It is well-known that the nonlinear multiplet possesses a formulation in terms of a harmonic-independent superfield $L^{\mathrm{a} i}$. In complex harmonic superspace, it is encoded as

$$
N^{(0,2)} \equiv \frac{L^{\mathrm{a}+} w_{\mathrm{a}}^{+}}{L^{\mathrm{b}+} w_{\mathrm{b}}^{-}}=\frac{L^{\mathrm{a} i} w_{\mathrm{a}}^{+} v_{i}^{+}}{L^{\mathrm{b} j} w_{\mathrm{b}}^{-} v_{j}^{+}} .
$$

One can easily confirm the constraints (3.30). In terms of the complex harmonic coordinates, this expression becomes

$$
N^{(0,2)}=z^{++}+\frac{L^{\mathrm{a} i} u_{\mathrm{a}}^{+} u_{i}^{+}}{L^{\mathrm{b} j} u_{\mathrm{b}}^{-} u_{j}^{+}} .
$$

The first term may be understood as generating the inhomogeneous term in (3.31). Following [2], we take $L^{\mathrm{a} i}$ to be normalized as $L^{\mathrm{a} i} L_{\mathrm{a} j}=\delta_{j}^{i}$ and $L^{\mathrm{a} i} L_{\mathrm{b} i}=\delta_{\mathrm{b}}^{\mathrm{a}}$ and raise/lower the indices in the same way. The analyticity condition and transformation rule becomes

$$
L^{\mathrm{a}(k} \nabla_{\underline{\alpha}}^{i} L_{\mathrm{a}}^{j)}=0, \quad \delta L^{\mathrm{a} i}=\xi^{A} \nabla_{A} L^{\mathrm{a} i}+\lambda^{i}{ }_{j} L^{\mathrm{a} j} .
$$

As with the relaxed hypermultiplet, these conditions indicate that the index $i$ of $L^{\text {ai }}$ is an $\mathrm{SU}(2)_{R}$ index, while a is an external index, consistent with (3.32). This form of the nonlinear multiplet frequently appears as a compensator in $\mathcal{N}=2$ supergravity [46, 49-51].

The $Q^{+}$hypermultiplet. Now we turn to the $Q^{+}$hypermultiplet, which is the general matter multiplet of harmonic superspace as well as the general compensating multiplet of supergravity [52]. In the complex harmonic description, it possesses charge $(1,0)$ under $\mathrm{U}(1)_{\mathrm{v}} \times \mathrm{U}(1)_{\mathrm{w}}$. Assuming that $Q^{+}$is twisted biholomorphic, it is easy to see that the charge assignments are consistent with the free hypermultiplet equation of motion $D_{\mathrm{w}}^{++} Q^{+}=0$ : in that case the free on-shell $Q^{+}$is an $\mathcal{O}(1)$ superfield. Because of the importance of this multiplet, we will make a few further comments that are obvious generalizations of 
its conventional description in harmonic superspace. Its general off-shell version can be expanded in the central basis as

$$
\begin{aligned}
Q^{+} & =\sum_{n=0}^{\infty} Q^{\left(i_{1} \cdots i_{n+1} j_{1} \cdots j_{n}\right)} v_{i_{1}}^{+} \cdots v_{i_{n+1}}^{+} w_{j_{1}}^{-} \cdots w_{j_{n}}^{-}\left(z^{0}\right)^{-n} \\
& =z^{0} \sum_{n=0}^{\infty} Q^{\left(i_{1} \cdots i_{n+1} j_{1} \cdots j_{n}\right)} u_{i_{1}}^{+} \cdots u_{i_{n+1}}^{+} u_{j_{1}}^{-} \cdots u_{j_{n}}^{-}
\end{aligned}
$$

We have not attempted here to maintain any distinction between the $\mathrm{SU}(2)_{\mathrm{v}}$ and $\mathrm{SU}(2)_{\mathrm{w}}$ indices because the presence of the $z^{0}$ factors renders the distinction meaningless; the various terms in this expansion will mix under $\mathrm{SU}(2)_{R}$. To see this, we note that the transformation property of $Q^{+}$may be written

$$
\delta Q^{+}=\xi^{A} \nabla_{A} Q^{+}+\Lambda_{\mathrm{D}} Q^{+}+\lambda_{\mathrm{u}}^{0} Q^{+}-\lambda_{\mathrm{u}}^{++} \partial_{\mathrm{u}}^{--} Q^{+} .
$$

This implies for the leading term $Q^{i}$ in (3.35) the transformation

$$
\delta Q^{i}=\xi^{A} \nabla_{A} Q^{i}+\Lambda_{\mathrm{D}} Q^{i}+\lambda^{i}{ }_{j} Q^{j}-\frac{1}{2} \lambda^{j k} Q_{j k}{ }^{i} .
$$

For the free on-shell hypermultiplet, all the higher terms vanish, leaving an $\mathcal{O}(1)$ multiplet.

The $\boldsymbol{\omega}$ hypermultiplet. The $\omega$ hypermultiplet is a variant version of the hypermultiplet, which can take several forms. We discuss here its simplest version, which can be constructed in conventional harmonic superspace from a pseudoreal doublet $Q^{\mathrm{a}+}=\left(Q^{+}, \widetilde{Q}^{+}\right)$involving a $Q^{+}$hypermultiplet and its conjugate $\widetilde{Q}^{+}$as $\omega=u_{\mathrm{a}}^{-} Q^{\mathrm{a}+}$. If $Q^{+}$is free, then $\omega$ obeys the free equation of motion $\left(D^{++}\right)^{2} \omega=0$ and corresponds to a relaxed hypermultiplet of Weyl weight 1.

In complex harmonic superspace, this version of $\omega$ becomes a twisted biholomorphic analytic superfield $\omega^{(1,-1)}$. Its $\mathrm{U}(1)_{\mathrm{v}}$ charge is implied by its Weyl weight, while its $\mathrm{U}(1)_{\mathrm{w}}$ charge is implied if we assume that the free equation of motion should be $\left(D_{\mathrm{w}}^{++}\right)^{2} \omega^{(1,-1)}=0$. Each of these properties is consistent with the choice $\omega^{(1,-1)}=w_{\mathrm{a}}^{-} Q^{\mathrm{a}+}$. Such a multiplet is manifestly twisted biholomorphic with each of the requisite weights. The general $\omega^{(1,-1)}$ hypermultiplet transforms as

$$
\delta \omega^{(1,-1)}=\xi^{A} \nabla_{A} \omega^{(1,-1)}-\lambda_{\mathrm{u}}^{++} \partial_{\mathrm{u}}^{--} \omega^{(1,-1)}+\left(\Lambda_{\mathrm{D}}+\lambda_{\mathrm{u}}^{0}\right) \omega^{(1,-1)} .
$$

Abelian vector multiplet. Finally, we turn to the vector multiplet. For simplicity, our attention here will be on the abelian case, but the non-abelian version is a straightforward extension. Recall that the abelian vector multiplet is described by an analytic prepotential $V^{++}$constructed in terms of a bridge superfield $B$ via $V^{++}=D^{++} B$. The bridge $B$ is globally defined on $S^{2}$ but not analytic. Both the bridge and $V^{++}$must have vanishing Weyl weight.

In complex harmonic superspace, the vector multiplet is described by a twisted biholomorphic analytic superfield $V^{(0,2)}$. Its $\mathrm{U}(1)_{\mathrm{v}}$ charge must vanish, consistent with its Weyl weight. Obviously, $V^{(0,2)}$ should be interpreted as a connection for the complex harmonic 
derivative $\nabla_{\mathrm{w}}^{++}$in the analytic basis. This implies that it should be related to a bridge superfield $B$ via $V^{(0,2)}=D_{\mathrm{w}}^{++} B$. The bridge $B$ should be twisted biholomorphic with vanishing harmonic charges. To confirm this interpretation, we note that the transformation of $V^{(0,2)}$ reproduces the transformation in conventional harmonic superspace,

$$
\delta V^{(0,2)}=\xi^{A} \nabla_{A} V^{(0,2)}-\lambda_{\mathrm{v}}^{++} D_{\mathrm{v}}^{--} V^{(0,2)}=\xi^{A} \nabla_{A} V^{(0,2)}-\lambda_{\mathrm{u}}^{++} \partial_{\mathrm{u}}^{--} V^{(0,2)} .
$$

In contrast, we expect $V^{--} \equiv V^{(-2,0)}$ to be the connection for the complex harmonic derivative $\nabla_{\mathrm{v}}^{--}$in the analytic basis and given by $V^{(-2,0)}=D_{\mathrm{v}}^{--} B$. It is easy to check that $V^{(-2,0)}$ is also twisted biholomorphic, though it is not analytic. It transforms as

$$
\begin{aligned}
\delta V^{(-2,0)} & =\xi^{A} \nabla_{A} V^{(-2,0)}-\lambda_{\mathrm{v}}^{++} D_{\mathrm{v}}^{--} V^{(-2,0)}-2 \lambda_{\mathrm{v}}^{0} V^{(-2,0)} \\
& =\xi^{A} \nabla_{A} V^{(-2,0)}-\lambda_{\mathrm{u}}^{++} \partial_{\mathrm{u}}^{--} V^{(-2,0)}-2 \lambda_{\mathrm{u}}^{0} V^{(-2,0)}
\end{aligned}
$$

The differences in the covariant transformation laws for $V^{(-2,0)}$ and $V^{(0,2)}$ are naturally explained by their $\mathrm{U}(1)_{\mathrm{v}} \times \mathrm{U}(1)_{\mathrm{w}}$ charges in the complex harmonic approach.

\section{Superspace action principles on $\mathcal{M}^{4 \mid 8} \times \mathrm{SU}(2)_{\mathrm{v}} \times \mathrm{SU}(2)_{\mathrm{w}}$}

In this section, we will address both the full harmonic superspace and analytic superspace actions, discuss how to relate one to the other, and provide the component reduction formula for the analytic action. A few specific examples will also be discussed.

\subsection{Full superspace}

The natural twisted biholomorphic integral over full superspace is given by

$$
\frac{i}{2 \pi} \int \mathrm{d}^{4} x \mathrm{~d}^{4} \theta \mathrm{d}^{4} \bar{\theta} E \int_{\mathcal{S}} \mathcal{V}^{++} \wedge \mathcal{W}^{--} \mathscr{L}^{(-2,2)},
$$

where the first integral is evaluated in the central basis and the second is over the closed surface $\mathcal{S}$ homotopic to the real $S^{2}$. The harmonic charges of the Lagrangian are chosen to counter the measure factor. The Lagrangian must have vanishing dilatation and $\mathrm{U}(1)_{R}$ weights and be a conformal primary. This expression can be generalized to any gauge,

$$
\frac{i}{2 \pi} \int_{\mathcal{S}} \mathrm{d}^{2} \zeta \int \mathrm{d}^{4} x \mathrm{~d}^{4} \theta^{+} \mathrm{d}^{4} \theta^{-} E^{(2,-2)} \mathscr{L}^{(-2,2)}
$$

with the measure

$$
E^{(2,-2)}=\operatorname{sdet}\left(\begin{array}{ccc}
E_{M^{A}} & E_{M^{\mathrm{v}++}} & E_{M^{\mathrm{w}}--} \\
E_{\zeta}^{A} & E_{\zeta^{\mathrm{v}}++} & E_{\zeta^{\mathrm{w}}--} \\
E_{\tilde{\zeta}}^{A} & E_{\tilde{\zeta}^{\mathrm{v}++}} & E_{\tilde{\zeta}^{\mathrm{w}--}}
\end{array}\right) .
$$

The complex coordinates $\zeta$ and $\tilde{\zeta}$ parametrize $\mathcal{S}$ with $(\zeta)^{*} \neq \tilde{\zeta}$ in general, and $E_{\zeta}{ }^{\mathcal{A}}$ and $E_{\tilde{\zeta}} \mathcal{A}$ are the pullback of the vielbein. The charge assignments of the measure $E^{(2,-2)}$ correspond to its weight under covariant $\nabla_{\mathrm{v}}^{0}$ and $\nabla_{\mathrm{w}}^{0}$ diffeomorphisms. We have written the Grassmann coordinates suggestively as $\theta^{+}$and $\theta^{-}$, but in a general gauge they possess no meaningful charge or relation to the harmonics. Using e.g. the results of appendix 
B of [10] (see the summary in appendix A) one may confirm that (4.2) is a gauge and diffeomorphism-invariant quantity.

It is useful to know when a quantity is a total derivative in the covariant approach. In appendix A, we show that the covariant expression $\mathscr{L}^{(-2,2)}=\nabla_{\mathrm{w}}^{++} V^{(-2,0)}+\nabla_{\mathrm{v}}^{--} V^{(0,2)}$ is a total derivative for any twisted biholomorphic conformal primary superfields $V^{(-2,0)}$ and $V^{(0,2)}$ with vanishing Weyl and $\mathrm{U}(1)_{R}$ weights. Other expressions which appear to be total derivatives such as $\nabla_{A} V^{A}$ generally fail to be primary - and so are not permitted as covariant integrands - or leave residual connections when integrated by parts.

\subsection{Analytic superspace}

We will be particularly interested in the action principle for analytic superspace. In the analytic basis, its form is well-known:

$$
\int_{S^{2}} \mathrm{~d} u \int \mathrm{d}^{4} \hat{x} \mathrm{~d}^{4} \hat{\theta}^{+} \hat{\mathscr{L}}^{+4}
$$

The analytic Lagrangian $\hat{\mathscr{L}}^{+4}$ must transform as a scalar density, ensuring that the action is invariant under analytic gauge transformations. The integration is performed over the real $S^{2}$ manifold. To describe the same action principle in a general gauge, we propose

$$
\frac{i}{2 \pi} \int_{\mathcal{S}} \mathrm{d}^{2} \zeta \int \mathrm{d}^{4} x \mathrm{~d}^{4} \theta^{+} \mathcal{E}^{(-2,-2)} \mathscr{L}^{(2,2)}
$$

The Lagrangian $\mathscr{L}^{(2,2)}$ is now a scalar function rather than a scalar density and the analytic measure is given by

$$
\mathcal{E}^{(-2,-2)}=\operatorname{sdet}\left(\begin{array}{cccc}
E_{m}{ }^{a} & E_{m}{ }^{\underline{\alpha}+} & E_{m}{ }^{\mathrm{v}++} & E_{m}{ }^{\mathrm{w}--} \\
E_{\underline{\mu}+}{ }^{a} & E_{\underline{\mu}+}{ }^{\underline{\alpha}+} & E_{\underline{\mu}+}{ }^{\mathrm{v}++} & E_{\underline{\mu}+}{ }^{\mathrm{w}--} \\
E_{\zeta}{ }^{a} & E_{\zeta} \underline{\alpha}+ & E_{\zeta}{ }^{\mathrm{v}++} & E_{\zeta^{\mathrm{w}}--} \\
E_{\tilde{\zeta}}{ }^{a} & E_{\tilde{\zeta}}{ }^{\underline{\alpha}+} & E_{\tilde{\zeta}^{\mathrm{v}}++} & E_{\tilde{\zeta}^{\mathrm{w}}--}
\end{array}\right) .
$$

We have labeled the measure again with its weights under covariant $\nabla_{\mathrm{v}}^{0}$ and $\nabla_{\mathrm{w}}^{0}$ diffeomorphisms, and we reiterate that the charge assignment of the Grassmann coordinates is not meaningful in a general gauge. Later on, when we return to the analytic basis, we will find a different notion of charge for these objects that concurs with (4.4). One can show that (4.5) is gauge-invariant provided $\mathscr{L}^{(2,2)}$ is an analytic twisted biholomorphic conformal primary of Weyl weight two. To establish the equivalence of (4.4) and (4.5) requires a more elaborate discussion, which will be postponed until section 5 .

It is a straightforward exercise (by e.g. generalizing the argument in [10]) to show that any full superspace integral (4.2) can be written as an analytic superspace integral

$$
\frac{i}{2 \pi} \int_{\mathcal{S}} \mathrm{d}^{2} \zeta \int \mathrm{d}^{4} x \mathrm{~d}^{8} \theta E^{(2,-2)} \mathscr{L}^{(-2,2)}=\frac{i}{2 \pi} \int_{\mathcal{S}} \mathrm{d}^{2} \zeta \int \mathrm{d}^{4} x \mathrm{~d}^{4} \theta^{+} \mathcal{E}^{(-2,-2)}\left(\nabla^{+}\right)^{4} \mathscr{L}^{(-2,2)} .
$$

The superfield $\left(\nabla^{+}\right)^{4} \mathscr{L}^{(-2,2)}$ obeys all the requirements of an analytic Lagrangian $\mathscr{L}^{(2,2)}$. Similarly, one can lift any analytic action to a full superspace action. Following the same 
procedure as in [10], we introduce a real nowhere-vanishing harmonic-independent superfield $\Omega$ of Weyl weight two, writing

$$
\frac{i}{2 \pi} \int_{\mathcal{S}} \mathrm{d}^{2} \zeta \int \mathrm{d}^{4} x \mathrm{~d}^{4} \theta^{+} \mathcal{E}^{(-2,-2)} \mathscr{L}^{(2,2)}=\frac{i}{2 \pi} \int_{\mathcal{S}} \mathrm{d}^{2} \zeta \int \mathrm{d}^{4} x \mathrm{~d}^{8} \theta E^{(2,-2)} \frac{\Omega}{\left(\nabla^{+}\right)^{4} \Omega} \mathscr{L}^{(2,2)}
$$

Now let us choose $\Omega=W \bar{W}$ for a vector multiplet $W$ and then adopt the gauge where $W=1$. This effects the conversion of conformal superspace [45] to $\mathrm{SU}(2)$ superspace $[21,53]$ and lets one rewrite (4.8) as

$$
\frac{i}{2 \pi} \int_{\mathcal{S}} \mathrm{d}^{2} \zeta \int \mathrm{d}^{4} x \mathrm{~d}^{8} \theta \frac{E^{(2,-2)}}{\left(S^{++}\right)^{2}} \mathscr{L}^{(2,2)}=\frac{i}{2 \pi} \int \mathrm{d}^{4} x \mathrm{~d}^{8} \theta E \int_{\mathcal{S}} \mathcal{V}^{++} \wedge \mathcal{W}^{--} \frac{\mathscr{L}^{(2,2)}}{\left(S^{++}\right)^{2}},
$$

where $S^{++}$is a torsion superfield of $\mathrm{SU}(2)$ superspace and the right-hand side is written in the central basis. If we restrict to the real $S^{2}$, this simplifies still further to

$$
\int \mathrm{d}^{4} x \mathrm{~d}^{8} \theta E \int_{S^{2}} \mathrm{~d} u \frac{1}{\left(S^{++}\right)^{2}} \mathscr{L}^{+4}
$$

This is a convenient formulation of curved harmonic superspace in the central basis using $\mathrm{SU}(2)$ superspace and is inspired by an analogous formula in projective superspace [21].

In appendix A, we briefly discuss how to show that $\mathscr{L}^{(2,2)}=\nabla_{\mathrm{w}}^{++} V^{(2,0)}$ is a total derivative when $V^{(2,0)}$ is an analytic twisted biholomorphic conformal primary. As an exercise, one may show this by introducing a prepotential for $V^{(2,0)}$ as $V^{(2,0)}=\left(\nabla^{+}\right)^{4} V^{(-2,0)}$ and then observing that $\nabla_{\mathrm{w}}^{++} V^{(-2,0)}$ is a total derivative as a full superspace Lagrangian. Note that a similar quantity, $\nabla_{\mathrm{v}}^{--} V^{(4,2)}$, is not an allowed analytic Lagrangian as it is not twisted biholomorphic.

\subsection{Analytic superspace component action}

Now we turn to deriving the component form of the analytic superspace action (4.5). Upon integration over the Grassmann coordinates, the final form of the action should be

$$
S=\frac{i}{2 \pi} \int_{\mathcal{M}^{4} \times \mathcal{S}} \mathcal{J}
$$

for some closed six-form $\mathcal{J}$ integrated over the product of $4 D$ spacetime $\mathcal{M}^{4}$ and the auxiliary manifold $\mathcal{S}$. Here it helps to recall the projective superspace result [10], where

$$
-\frac{1}{2 \pi} \oint_{\mathcal{C}} \mathrm{d} \tau \int \mathrm{d}^{4} x \mathrm{~d}^{4} \theta^{+} \mathcal{E}^{--} \mathscr{L}^{++}=-\frac{1}{2 \pi} \int_{\mathcal{M}^{4} \times \mathcal{C}} \mathcal{J}_{P}
$$

in terms of a five-form $\mathcal{J}_{P}$, which was quite complicated in a general gauge. Its leading term was

$$
\mathcal{J}_{P}=e^{0} \wedge e^{1} \wedge e^{2} \wedge e^{3} \wedge e^{\mathrm{v}++}\left(\nabla^{-}\right)^{4} \mathscr{L}^{++} \mid+\cdots
$$

with the subleading terms each involving a five-form multiplied by a certain number of covariant derivatives of $\mathscr{L}^{++}$. Here we have written $e^{a}=E^{a} \|$ and $e^{\mathrm{v}++}=E^{\mathrm{v}++} \|$ as the double-bar projections (setting $\theta=\mathrm{d} \theta=0$ ) of the corresponding vielbeins. Keeping 
in mind that complex harmonic superspace can be understood as projective superspace combined with an additional $\mathbb{C} P^{1}$ manifold, one can make the guess that $\mathcal{J}$ should be given by inserting the harmonic Lagrangian into $\mathcal{J}_{P}$ and taking the wedge product with $e^{\mathrm{w}--}=E^{\mathrm{w}--} \|$, that is,

$$
\mathcal{J}=\mathcal{J}_{P}\left[\mathscr{L}^{(2,2)}\right] \wedge e^{\mathrm{w}--}=e^{0} \wedge e^{1} \wedge e^{2} \wedge e^{3} \wedge e^{\mathrm{v}++} \wedge e^{\mathrm{w}--}\left(\nabla^{-}\right)^{4} \mathscr{L}^{(2,2)} \mid+\cdots
$$

This turns out to be the correct answer.

There are two approaches to checking this result. The first is simply to repeat the normal coordinate calculation given in appendix $\mathrm{C}$ of [10]. The main difference is that one encounters the volume six-form $\hat{e}^{(2,-2)}=\hat{e}^{++} \wedge e^{\mathrm{w}--}$ where $\hat{e}^{++}$is the volume fiveform when restricted to the bosonic body $\mathcal{M}^{4} \times \mathbb{C} P^{1}$ of projective superspace. Viewed as a superform, its $\theta^{+}$expansion is responsible for giving the subleading terms in the component action. But because $e^{\mathrm{w}--}$ has a trivial $\theta^{+}$expansion when written in Grassmann normal coordinates, no new features are encountered. This reproduces (4.14).

A less direct approach is to observe that $\mathcal{J}$ must be a closed six-form in superspace. Because of the twisted biholomorphic nature of $\mathscr{L}^{(2,2)}$, one can show that the closure of $\mathcal{J}_{P}$, interpreted as a five-form in projective superspace, implies the closure of $\mathcal{J}$. One observes for the leading term of $\mathcal{J}_{P}$ (and similarly for the subleading terms)

$$
0=\mathrm{d} \mathcal{J}_{P}=\mathrm{d} \hat{e}^{++}\left(\nabla^{-}\right)^{4} \mathscr{L}^{++}+\hat{e}^{++} \wedge\left(E^{A} \nabla_{A}+E^{\mathrm{v} \underline{a}} \nabla_{\mathrm{v} \underline{a}}+\cdots\right)\left(\nabla^{-}\right)^{4} \mathscr{L}^{++}+\cdots .
$$

The second term in parentheses is the expansion of the exterior derivative in projective superspace; we have exhibited the vielbeins but suppressed the other connections. When we formally replace $\mathscr{L}^{++}$with $\mathscr{L}^{(2,2)}$, the second expression turns out to be missing the $\mathrm{SU}(2)_{\mathrm{w}}$ vielbeins. Adding and subtracting these gives

$$
\begin{aligned}
0= & \mathrm{d} \hat{e}^{++}\left(\nabla^{-}\right)^{4} \mathscr{L}^{(2,2)}+\hat{e}^{++} \wedge\left(E^{A} \nabla_{A}+E^{\mathrm{v} \underline{a}} \nabla_{\mathrm{v} \underline{a}}+E^{\mathrm{w} \bar{a}} \nabla_{\mathrm{w} \bar{a}}+\cdots\right)\left(\nabla^{-}\right)^{4} \mathscr{L}^{(2,2)} \\
& -2 \hat{e}^{++} \wedge E^{\mathrm{w} 0}\left(\nabla^{-}\right)^{4} \mathscr{L}^{(2,2)}-\hat{e}^{++} \wedge E^{\mathrm{w}--} \nabla_{\mathrm{w}}^{++}\left(\nabla^{-}\right)^{4} \mathscr{L}^{(2,2)}+\cdots .
\end{aligned}
$$

The first line is the exterior derivative of the leading term of $\mathcal{J}_{P}\left[\mathscr{L}^{(2,2)}\right]$. Taking the wedge product with $E^{\mathrm{w}--}$ gives for this leading term

$$
0=\mathrm{d} \mathcal{J}_{P}\left[\mathscr{L}^{(2,2)}\right] \wedge E^{\mathrm{w}--}-2 \mathcal{J}_{P}\left[\mathscr{L}^{(2,2)}\right] \wedge E^{\mathrm{w} 0} \wedge E^{\mathrm{w}--}=-\mathrm{d} \mathcal{J}
$$

and so $\mathcal{J}$ is closed. The subleading terms more or less go the same way. A similar line of argument shows that if $\mathcal{J}_{P}$ is gauge-invariant in projective superspace up to an exact form, then so is $\mathcal{J}$ in harmonic superspace.

It should go without saying that the central basis is to be preferred for component actions. In that gauge, one finds

$$
S=\int \mathrm{d}^{4} x \text { e } \mathcal{L}, \quad \mathcal{L}=\frac{i}{2 \pi} \int_{\mathcal{S}}\left(\mathcal{V}^{++} \wedge \mathcal{W}^{--} \mathcal{L}^{(-2,2)}-\mathcal{V}^{--} \wedge \mathcal{W}^{--} \mathcal{L}^{(2,2)}\right)
$$

where $\mathcal{L}^{(-2,2)}$ and $\mathcal{L}^{(2,2)}$ coincide with the component Lagrangians given in [10] with the replacement $\mathscr{L}^{++} \rightarrow \mathscr{L}^{(2,2)}$. They are (with projection to $\theta=0$ understood)

$$
\mathcal{L}^{(-2,2)}=\frac{1}{16}\left(\nabla^{-}\right)^{2}\left(\bar{\nabla}^{-}\right)^{2} \mathscr{L}^{(2,2)}-\frac{i}{8}\left(\bar{\psi}_{m}^{-} \bar{\sigma}^{m}\right)^{\alpha} \nabla_{\alpha}^{-}\left(\bar{\nabla}^{-}\right)^{2} \mathscr{L}^{(2,2)}-\frac{i}{8}\left(\psi_{m}^{-} \sigma^{m}\right)_{\dot{\alpha}} \bar{\nabla}^{\dot{\alpha}-}\left(\nabla^{-}\right)^{2} \mathscr{L}^{(2,2)}
$$




$$
\begin{aligned}
& +\frac{1}{4}\left(\left(\psi_{n}^{-} \sigma^{n m}\right)^{\alpha} \bar{\psi}_{m}{ }^{\dot{\alpha}-}+\psi_{n}{ }^{\alpha-}\left(\bar{\sigma}^{n m} \bar{\psi}_{m}^{-}\right)^{\dot{\alpha}}-i \mathcal{V}_{m}^{--} \sigma_{\alpha \dot{\alpha}}^{m}\right)\left[\nabla_{\alpha}^{-}, \bar{\nabla}_{\dot{\alpha}}^{-}\right] \mathscr{L}^{(2,2)} \\
& +\frac{1}{4}\left(\psi_{m}^{-} \sigma^{m n} \psi_{n}^{-}\right)\left(\nabla^{-}\right)^{2} \mathscr{L}^{(2,2)}+\frac{1}{4}\left(\bar{\psi}_{m}^{-} \bar{\sigma}^{m n} \bar{\psi}_{n}^{-}\right)\left(\bar{\nabla}^{-}\right)^{2} \mathscr{L}^{(2,2)} \\
& -\left(\frac{1}{2} \epsilon^{m n p q}\left(\psi_{m}^{-} \sigma_{n} \bar{\psi}_{p}^{-}\right) \psi_{q}^{\alpha-}-2\left(\psi_{m}^{-} \sigma^{m n}\right)^{\alpha} \mathcal{V}_{n}^{--}\right) \nabla_{\alpha}^{-} \mathscr{L}^{(2,2)} \\
& +\left(\frac{1}{2} \epsilon^{m n p q}\left(\bar{\psi}_{m}^{-} \bar{\sigma}_{n} \psi_{p}^{-}\right) \bar{\psi}_{q \dot{\alpha}}^{-}-2\left(\bar{\psi}_{m}^{-} \bar{\sigma}^{m n}\right)_{\dot{\alpha}} \mathcal{V}_{n}^{--}\right) \bar{\nabla}^{\dot{\alpha}-} \mathscr{L}^{(2,2)} \\
& +3 \epsilon^{m n p q}\left(\psi_{m}^{-} \sigma_{n} \bar{\psi}_{p}^{-}\right) \mathcal{V}_{q}^{--} \mathscr{L}^{(2,2)}
\end{aligned}
$$

and

$$
\begin{aligned}
\mathcal{L}^{(2,2)}= & -\left[3 D+\frac{3 i}{2}\left(\bar{\psi}_{m}^{-} \bar{\sigma}^{m} \chi^{+}\right)-\frac{3 i}{2}\left(\psi_{m}^{-} \sigma^{m} \bar{\chi}^{+}\right)+4 f_{a}^{a}\right. \\
& \left.-4\left(\bar{\psi}_{m}^{-} \bar{\sigma}^{m n} \bar{\phi}_{n}^{+}\right)+4\left(\psi_{m}^{-} \sigma^{m n} \phi_{n}^{+}\right)-3 \epsilon^{m n p q}\left(\psi_{m}^{-} \sigma_{n} \bar{\psi}_{p}^{-}\right) \mathcal{V}_{q}^{++}\right] \mathscr{L}^{(2,2)} \\
+ & {\left[\frac{3}{2} \chi^{\alpha+}-i\left(\bar{\phi}_{m}^{+} \bar{\sigma}^{m}\right)^{\alpha}+2\left(\psi_{m}^{-} \sigma^{m n}\right)^{\alpha} \mathcal{V}_{n}^{++}\right] \nabla_{\alpha}^{-} \mathscr{L}^{(2,2)} } \\
- & {\left[\frac{3}{2} \chi_{\dot{\alpha}}^{+}-i\left(\phi_{m}^{+} \sigma^{m}\right)_{\dot{\alpha}}+2\left(\bar{\psi}_{m}^{-} \bar{\sigma}^{m n}\right)_{\dot{\alpha}} \mathcal{V}_{n}^{++}\right] \bar{\nabla}^{\dot{\alpha}-} \mathscr{L}^{(2,2)} } \\
& -\frac{i}{4} \mathcal{V}_{m}^{++}\left(\bar{\sigma}^{m}\right)^{\dot{\alpha} \alpha}\left[\nabla_{\alpha}^{-}, \bar{\nabla}_{\dot{\alpha}}^{-}\right] \mathscr{L}^{(2,2)} .
\end{aligned}
$$

Above we have the component fields as defined in [45] corresponding to the content of $\mathcal{N}=2$ conformal supergravity. These consist of (i) the five fundamental connections the vierbein $e_{m}{ }^{a}$, the gravitini $\psi_{m}{ }^{\alpha}{ }_{i}$, the $\mathrm{SU}(2)_{\mathrm{R}}$ and $\mathrm{U}(1)_{\mathrm{R}}$ connections $\mathcal{V}_{m}{ }^{i}{ }_{j}$ and $A_{m}$, and the dilatation connection $b_{m}$; (ii) covariant auxiliary fields $W_{a b}=\frac{1}{4} T_{a b}^{-}, \chi_{\alpha i}$, and $D$; and (iii) composite connections $\omega_{m}{ }^{a b}, \phi_{m}{ }^{\alpha i}$ and $f_{m}{ }^{a}$, given in terms of the other fields, which are associated with Lorentz, $S$-supersymmetry and special conformal gauge symmetries. In the expressions (4.19) and (4.20), these fields are contracted with $\mathrm{SU}(2)_{\mathrm{v}}$ harmonics, e.g. $\psi_{m}^{ \pm}=\psi_{m}^{i} v_{i}^{ \pm}, \chi^{ \pm}=\chi^{i} v_{i}^{ \pm}, \mathcal{V}_{m}^{ \pm \pm}=\mathcal{V}_{m}^{i j} v_{i}^{ \pm} v_{j}^{ \pm}$, and so forth.

Now observe that interchanging the order of integration gives

$$
\begin{aligned}
S & =\frac{i}{2 \pi} \int_{\mathcal{S}} \mathcal{V}^{++} \wedge \mathcal{W}^{--} \omega^{(-2,2)}-\frac{i}{2 \pi} \int_{\mathcal{S}} \mathcal{V}^{--} \wedge \mathcal{W}^{--} \omega^{(2,2)}, \\
\omega^{(-2,2)} & =\int \mathrm{d}^{4} x e \mathcal{L}^{(-2,2)}, \quad \omega^{(2,2)}=\int \mathrm{d}^{4} x e \mathcal{L}^{(2,2)} .
\end{aligned}
$$

The two-form $\omega=\mathcal{V}^{++} \wedge \mathcal{W}^{--} \omega^{(-2,2)}-\mathcal{V}^{--} \wedge \mathcal{W}^{--} \omega^{(2,2)}$ is of the type discussed in section 2: it is closed on $\mathcal{S}$ and ensures that the action is insensitive to small deformations of $\mathcal{S}$. We may then restrict to $\mathcal{S}=S^{2}$, where the second integral in (4.21) drops out, leaving the more conventional expression

$$
S=\int \mathrm{d}^{4} x e \int_{S^{2}} \mathrm{~d} u \mathcal{L}^{0}, \quad \mathcal{L}^{0}=\left.\mathcal{L}^{(-2,2)}\right|_{S^{2}}
$$

This yields the most compact form for the component Lagrangian of a general curved harmonic superspace action and constitutes another of our major results. 


\subsection{Examples of complex harmonic superspace actions}

Below we will briefly summarize how the most common harmonic superspace actions can be written in the covariant formulation here. Each of these cases is a straightforward extension of a well-known action in conventional harmonic superspace.

The $Q^{+}$hypermultiplet and general self-couplings. Introduce a family of hypermultiplets $Q^{\mathrm{a}+}$ with a $=1, \cdots, 2 n$, and impose a pseudoreality condition $\widetilde{Q^{\mathrm{a}+}}=-Q_{\mathrm{a}}^{+} \equiv$ $-Q^{\mathrm{b}+} \Omega_{\mathrm{ba}}$ using the canonical symplectic form $\Omega_{\mathrm{ba}}$ of $\operatorname{Sp}(n)$. Interactions may be introduced in the form of a twisted biholomorphic function $H^{(2,2)}$, so that the Lagrangian is

$$
\mathscr{L}^{(2,2)}=\frac{1}{2} Q_{\mathrm{a}}^{+} \nabla_{\mathrm{w}}^{++} Q^{\mathrm{a}+}+H^{(2,2)} .
$$

We have denoted the potential term by $H^{(2,2)}$ as in [36] to emphasize its interpretation as a Hamiltonian [54]. In flat space, it is natural to require $H^{(2,2)}=H^{(2,2)}\left(Q^{+}, v^{+}, w^{-}\right)$to be analytic and twisted biholomorphic; however, when coupled to conformal supergravity, it must have Weyl weight 2 and so

$$
Q^{\mathrm{a}+} \partial_{\mathrm{a}+} H^{(2,2)}=2 H^{(2,2)} \quad \Longrightarrow \quad H^{(2,2)}=H^{(2,2)}\left(Q^{+}, w^{-}\right) .
$$

In other words, the Hamiltonian must not explicitly depend on $v^{i+}[2,55]$. The component sigma model corresponding to this action describes a hyperkähler cone. The condition that $H^{(2,2)}$ cannot depend on $v^{i+}$ can also be interpreted as requiring the Lagrangian to be a scalar function under $\mathrm{SU}(2)_{\mathrm{v}}$ diffeomorphisms. A similar condition is required in projective superspace. In contrast, it is permissible for the $w_{i}^{-}$to appear because one may avoid ever using non-trivial $\mathrm{SU}(2)_{\mathrm{w}}$ diffeomorphisms.

The $\omega$ hypermultiplet action. The free $\omega$ hypermultiplet action can be constructed from the free $Q^{\mathrm{a}+}$ hypermultiplet action with a $=1,2$ by making the change of variables $Q^{\mathrm{a}+}=w^{\mathrm{a}+} \omega^{(1,-1)}-w^{\mathrm{a}-} f^{(1,1)}$. One must employ the $w_{i}^{ \pm}$harmonics so that $\omega^{(1,-1)}$ and $f^{(1,1)}$ remain covariant under $\mathrm{SU}(2)_{\mathrm{v}}$ diffeomorphisms. It is evident from this equation that $f^{(1,1)}$ is not a twisted biholomorphic superfield but rather obeys the constraint $\nabla_{\mathrm{w}}^{--} f^{(1,1)}=\omega^{(1,-1)}$. This can be remedied by replacing $f^{(1,1)} \rightarrow f^{(1,1)}+\nabla_{\mathrm{w}}^{++} \omega^{(1,-1)}$, so that the new fundamental superfields are each twisted biholomorphic. The Lagrangian remains twisted biholomorphic,

$$
\frac{1}{2} Q_{\mathrm{a}}^{+} \nabla_{\mathrm{w}}^{++} Q^{\mathrm{a}+}=\frac{1}{2} \omega^{(1,-1)}\left(\nabla_{\mathrm{w}}^{++}\right)^{2} \omega^{(1,-1)}+\frac{1}{2}\left(f^{(1,1)}\right)^{2}+\frac{1}{2} \nabla_{\mathrm{w}}^{++}\left(\omega^{(1,-1)} f^{(1,1)}\right) .
$$

The last term is a total derivative, and the second term can be integrated out, leaving the free $\omega$ hypermultiplet Lagrangian.

The improved tensor multiplet action. As our last example, let us generalize the construction of the improved tensor multiplet [56] to complex harmonic superspace. Starting with a free hypermultiplet action with a complex $Q^{+}$, one makes the complicated change of variables

$$
Q^{+}=e^{i \omega}\left(Q_{0}^{+}-i \frac{w_{\underline{2}}^{-}}{\Omega_{0}} g^{++}\right), \quad \widetilde{Q}^{+}=e^{-i \omega}\left(\widetilde{Q}_{0}^{+}-i \frac{w_{1}^{-}}{\Omega_{0}} g^{++}\right)
$$




$$
\Omega_{0} \equiv \Omega_{0}^{(1,-1)}:=Q_{0}^{+} w_{\underline{1}}^{-}+\widetilde{Q}_{0}^{+} w_{\underline{2}}^{-},
$$

where $\omega$ and $g^{++}$are two real dynamical variables and $Q_{0}^{+}$is a new complex hypermultiplet. None of the fields $\omega, g^{++}$, or $Q_{0}^{+}$carries $\mathrm{U}(1)_{\mathrm{w}}$ charge, and each is analytic, so $g^{++}$ must be Weyl weight two and $Q^{+}$Weyl weight one. However, because we have traded two real degrees of freedom for four, this must be a redundant description, independent of some combination of the new fields, which will become apparent in due course. It is convenient to group $Q_{0}^{+}$and $\widetilde{Q}_{0}^{+}$into the pseudoreal doublet $Q_{0}^{i+}=\left(Q_{0}^{+}, \widetilde{Q}_{0}^{+}\right)$, so that $\Omega_{0}$ can be written simply as $\Omega_{0}=Q_{0}^{i+} w_{i}^{-}$. Note that $\Omega_{0}$ is actually a weight $(1,-1)$ superfield, but we have suppressed the charges for notational simplicity.

The above construction differs in two ways from the rigid version given in [56]. First, that version would correspond to choosing a fixed $Q_{0}^{i+}=v^{i+}$. However, this choice is not possible in any gauge other than the analytic one, as it is generally inconsistent with the analytic condition $\nabla_{\underline{\alpha}}^{+} Q_{0}^{i+}=0$ because of the presence of the non-vanishing $\mathrm{SU}(2)_{\mathrm{v}}$ connection. ${ }^{8}$ The second difference is that the change of variables in [56] was more general, involving an isotriplet $c_{i j}$. The simplifying choice we have made corresponds to taking $c_{\underline{12}}=i / 2$ and $c_{11}=c_{\underline{22}}=0$, with the non-canonical normalization $c^{2}:=c^{i j} c_{i j} / 2=1 / 4$. Below we restore a more general $c_{i j}$ (but keeping this normalization).

After making the redefinition (4.26), the free hypermultiplet Lagrangian becomes

$$
\begin{aligned}
\mathscr{L}^{(2,2)}= & \frac{1}{2} \frac{\left(g^{++}\right)^{2}}{\Omega_{0}^{2}}-L^{++} \nabla_{\mathrm{w}}^{++} \omega+\frac{1}{2} Q_{0 i}^{+} \nabla_{\mathrm{w}}^{++} Q_{0}^{i+} \\
& -\frac{2 g^{++} c_{i j} w^{i-}}{\Omega_{0}} \nabla_{\mathrm{w}}^{++} Q_{0}^{j+}+\nabla_{\mathrm{w}}^{++}\left(g^{++} C^{+-}\right)
\end{aligned}
$$

where we have defined

$$
\begin{aligned}
C^{++} & :=c_{i j} Q_{0}^{i+} Q_{0}^{j+}, \quad C^{+-}:=c_{i j} Q_{0}^{i+} w^{j-} / \Omega_{0}, \quad C^{--}:=c_{i j} w^{i-} w^{j-} / \Omega_{0}^{2}, \\
L^{++} & :=C^{++}+g^{++}+C^{--} g^{++} .
\end{aligned}
$$

The last term in the Lagrangian is a total derivative and can be discarded. The second term, which involves $\omega$ as a Lagrange multiplier, sets $L^{++}$to be an $\mathcal{O}(2)$ multiplet. This determines $g^{++}$in the usual form

$$
g^{++}=\frac{2\left(L^{++}-C^{++}\right)}{1+\sqrt{1+4 C^{--}\left(L^{++}-C^{++}\right)}},
$$

in terms of which the Lagrangian can be written

$$
\mathscr{L}^{(2,2)}=\frac{1}{2} \frac{\left(g^{++}\right)^{2}}{\Omega_{0}^{2}}+\frac{1}{2} Q_{0 i}^{+} \nabla_{\mathrm{w}}^{++} Q_{0}^{i+}-2 \frac{g^{++} c_{i j} w^{i-}}{\Omega_{0}} \nabla_{\mathrm{w}}^{++} Q_{0}^{j+} .
$$

As already mentioned, the change of variables we have made is equivalent to the standard choice in harmonic superspace, except for the appearance of the new hypermultiplet

\footnotetext{
${ }^{8}$ Actually, the precise gauge choice made in conventional harmonic superspace is slightly different, because $Q_{0}^{i+}$ (like all analytic superfields) is chosen to be a scalar density.
} 
$Q_{0}^{i+}$ rather than its frozen value. It is possible to show that the action is actually independent of this hypermultiplet. The proof is equivalent to showing the conformal invariance of the conventional harmonic action. (The conformal invariance of (4.30) is manifest.) Under an arbitrary variation of $Q_{0}^{i+}$, one simply shows that $\mathscr{L}^{(2,2)}$ transforms as a total derivative,

$$
\delta \mathscr{L}^{(2,2)}=\nabla_{\mathrm{w}}^{++}\left(\frac{1}{2} Q_{0 i}^{+} \delta Q_{0}^{i+}-\frac{2}{\Omega_{0}} g^{++}\left(\delta Q_{0}^{i+} w_{i}^{-} C^{+-}-\delta Q_{0}^{i+} Q_{0 i}^{+} C^{--}\right)\right) .
$$

When constructing the component action, the multiplet $Q_{0}^{i+}$ must drop out.

The necessity of this additional hypermultiplet can also be understood by comparing the above construction to its curved projective superspace analogue where similar features occur [57]. Beginning with the free hypermultiplet Lagrangian $\mathscr{L}^{++}=i \Upsilon^{+} \breve{\Upsilon}^{+}$, with $\Upsilon^{+}$an arctic multiplet and $\breve{\Upsilon}^{+}$its antarctic conjugate, one introduces a redundant parametrization $\Upsilon^{+}=\Upsilon_{0}^{+} e^{\Lambda}$ analogous to (4.26), with $\Upsilon_{0}^{+}$an arbitrary weight-one arctic superfield and $\Lambda$ a weight-zero arctic superfield. The free hypermultiplet Lagrangian can be rewritten as

$$
\mathscr{L}^{++}=i \Upsilon_{0}^{+} \breve{\Upsilon}_{0}^{+} e^{\Lambda+\breve{\Lambda}}-L^{++}(\Lambda+\breve{\Lambda})
$$

after relaxing the requirement that $\Lambda+\breve{\Lambda}$ is the sum of an arctic and an antarctic superfield, enforcing it instead via the Lagrange multiplier $L^{++}$. Integrating out the unconstrained real analytic superfield $\Lambda+\breve{\Lambda}$, one finds the improved tensor Lagrangian

$$
\mathscr{L}^{++}=L^{++}-L^{++} \log \left(L^{++} / i \Upsilon_{0}^{+} \breve{\Upsilon}_{0}^{+}\right)
$$

It is easy to show that this is actually independent of the choice of $\Upsilon_{0}^{+}$in precise analogy to the spurious dependence of (4.30) on $Q_{0}^{i+} .{ }^{9}$ Its presence is necessary to ensure covariance of the Lagrangian, and in the appropriate analytic basis it can be set to a constant.

\section{The analytic basis and conventional harmonic superspace}

In the previous sections, we have constructed a covariant formulation of complex harmonic superspace on the supermanifold $\mathcal{M}^{4 \mid 8} \times \mathrm{SU}(2)_{\mathrm{v}} \times \mathrm{SU}(2)_{\mathrm{w}}$, with explicit gauging of the superconformal group. There is one major task which remains: we must explain how this formulation is related to the prepotential approach of [52] and the covariant formulation discussed in $[25,26]$. The connection with [52] is the easiest to elucidate as it arises naturally upon going to the analytic basis (or analytic gauge). After reviewing the analytic basis in the rigid limit, we will construct it for the general curved supermanifold. Afterwards, we will describe how to recover [52]: the key step will be to trade analytic scalar fields (which we have used up until now) for analytic scalar densities. In the final part of this section, we will sketch the relationship with $[25,26]$.

\subsection{Rigid harmonic superspace and its analytic basis}

Understanding the structure of the analytic basis requires a brief discussion of superconformal isometries. This material largely follows [2, 37] and is inspired by the related

\footnotetext{
${ }^{9}$ One method is to use the argument of [23] for the gauge invariance of the vector-tensor coupling.
} 
construction in projective superspace [24]. A corresponding discussion for the general class of $(\mathcal{N}, p, q)$ superspaces was given in $[8,9]$.

In flat $\mathcal{N}=2$ superspace, the superconformal transformation of any covariant superfield $\Psi$ is

$$
\delta \Psi=\xi^{A} D_{A} \Psi+\frac{1}{2} \lambda^{a b} M_{b a} \Psi+\Lambda_{\mathrm{D}} \mathbb{D} \Psi+\Lambda_{\mathrm{A}} \mathbb{A} \Psi+\lambda^{i}{ }_{j} I_{i}^{j} \Psi+\eta^{\underline{\alpha} i} S_{\underline{\alpha} i} \Psi+\epsilon^{a} K_{a} \Psi .
$$

We are interested only in transformations that preserve a rigid background, meaning that the fixed vielbeins and (vanishing) connections of flat $\mathcal{N}=2$ superspace must be preserved, $\left[\delta, D_{A}\right]=0$, from which a number of properties follow. The parameters $\xi^{A}$ describe superconformal Killing vectors, with $\xi^{a}$ obeying the so-called master equation

$$
D_{(\beta}^{i} \xi_{\alpha) \dot{\alpha}}=0, \quad \bar{D}_{i}^{(\dot{\beta}} \xi^{\dot{\alpha}) \alpha}=0
$$

The other quantities in (5.1) turn out to be derived from $\xi^{a}$ [24].

For a superfield $\Psi$ that depends on the harmonics $v_{i}^{ \pm}$and $w_{i}^{ \pm}$, the action of the $\mathrm{SU}(2)_{R}$ generators in the central basis is given by

$$
\lambda^{i}{ }_{j} I^{j}{ }_{i} \Psi=-\lambda_{\mathrm{v}}^{++} D_{\mathrm{v}}^{--} \Psi+\lambda_{\mathrm{v}}^{0} D_{\mathrm{v}}^{0} \Psi+\lambda_{\mathrm{v}}^{--} D_{\mathrm{v}}^{++} \Psi,
$$

with $\lambda_{\mathrm{v}}^{ \pm \pm}:=\lambda^{i j} v_{i}^{ \pm} v_{j}^{ \pm}$and $\lambda_{\mathrm{v}}^{0}:=\lambda^{i j} v_{i}^{+} v_{j}^{-}$. The most important example is an analytic twisted biholomorphic conformal primary $\mathcal{F}^{(n, m)}$, whose full transformation law is

$$
\delta \mathcal{F}^{(n, m)}=\xi^{a} D_{a} \mathcal{F}^{(n, m)}-\xi^{\underline{\alpha}+} D_{\underline{\alpha}}^{-} \mathcal{F}^{(n, m)}-\lambda_{\mathrm{v}}^{++} D_{\mathrm{v}}^{--} \mathcal{F}^{(n, m)}+n\left(\Lambda_{\mathrm{D}}+\lambda_{\mathrm{v}}^{0}\right) \mathcal{F}^{(n, m)},
$$

where $\xi^{\underline{\alpha} \pm}=v_{i}^{ \pm} \xi^{\underline{\alpha}}$ and $D_{\underline{\alpha}}^{ \pm}:=v_{i}^{ \pm} D_{\underline{\alpha}}{ }^{i}$. In order for $\delta \mathcal{F}^{(n, m)}$ to be analytic, one must have

$$
\bar{D}^{\dot{\alpha}+} \xi^{b}=2 i\left(\bar{\sigma}^{b}\right)^{\dot{\alpha} \alpha} \xi_{\alpha}^{+}, \quad D_{\alpha}^{+} \xi^{\beta+}=\delta_{\alpha}{ }^{\beta} \lambda_{\mathrm{v}}^{++}, \quad \bar{D}_{\dot{\alpha}}^{+} \xi^{\beta+}=0, \quad D_{\alpha}^{+}\left(\Lambda_{\mathrm{D}}+\lambda_{\mathrm{v}}^{0}\right)=0,
$$

which are consequences of the master equation. The analytic quantity $\Lambda:=\Lambda_{\mathrm{D}}+\lambda_{\mathrm{v}}^{0}$ may also be written

$$
\Lambda=\frac{1}{2}\left(D_{a} \xi^{a}+D_{\underline{\alpha}}^{-} \xi^{\underline{\alpha}+}-D_{\mathrm{v}}^{--} \lambda_{\mathrm{v}}^{++}\right) .
$$

Let us now introduce the analytic basis of rigid complex harmonic superspace. We choose complex harmonic coordinates $u^{i \pm}, z^{ \pm \pm}$and $z^{0}$ defined as in section 2 and take

$$
\begin{aligned}
& \hat{\theta}_{\underline{\alpha}}^{+}=\frac{\theta_{\underline{\alpha}}^{i} v_{i}^{+}}{\left(v^{+}, w^{-}\right)}=\theta_{\underline{\alpha}}^{i} u_{i}^{+}, \quad \hat{\theta}_{\underline{\alpha}}^{-}=\theta_{\underline{\alpha}}^{i} w_{i}^{-}=\theta_{\underline{\alpha}}^{i} u_{i}^{-}, \\
& \hat{x}^{m}=x^{m}-2 i \theta^{(i} \sigma^{m} \bar{\theta}^{j)} \frac{v_{i} \bar{w}_{j}}{(v, \bar{w})}=x^{m}-2 i \theta^{(i} \sigma^{m} \bar{\theta}^{j)} u_{i}^{+} u_{j}^{-} .
\end{aligned}
$$

The coordinates $\hat{x}^{m}, \hat{\theta}_{\alpha}^{+}$, and $u^{ \pm}$parametrize the analytic superspace in the analytic basis. Note that the Grassmann coordinates carry $\mathrm{U}(1)_{\mathrm{w}}$ charge, whereas the spinor derivatives carry $\mathrm{U}(1)_{\mathrm{v}}$ charge. In this coordinate system, the superspace derivatives become

$$
D_{a}=\hat{\partial}_{m}, \quad D_{\underline{\alpha}}^{+}=z_{0} \frac{\partial}{\partial \hat{\theta}^{\underline{\alpha}}-}
$$




$$
\begin{aligned}
& D_{\alpha}^{-}=-\frac{1}{z^{0}}\left(\frac{\partial}{\partial \hat{\theta}^{\alpha+}}-2 i\left(\sigma^{m} \hat{\bar{\theta}}^{-}\right)_{\alpha} \hat{\partial}_{m}-z^{--} \frac{\partial}{\partial \hat{\theta}^{\alpha-}}\right), \\
& \bar{D}_{\dot{\alpha}}^{-}=-\frac{1}{z^{0}}\left(\frac{\partial}{\partial \hat{\bar{\theta}}^{\dot{\alpha}+}}+2 i\left(\hat{\theta}^{-} \sigma^{m}\right)_{\dot{\alpha}} \hat{\partial}_{m}-z^{--} \frac{\partial}{\partial \hat{\theta}^{\dot{\alpha}-}}\right) .
\end{aligned}
$$

Three of the harmonic derivatives remain quite simple,

$$
D_{\mathrm{v}}^{++}=\left(z^{0}\right)^{2} \frac{\partial}{\partial z^{--}}, \quad D_{\mathrm{v}}^{0}=z^{0} \frac{\partial}{\partial z^{0}}, \quad D_{\mathrm{w}}^{--}=\frac{\partial}{\partial z^{++}},
$$

with the others are moderately more complicated,

$$
\begin{aligned}
& D_{\mathrm{w}}^{++}=\partial_{\mathrm{u}}^{++}-2 i\left(\hat{\theta}^{+} \sigma^{m} \hat{\bar{\theta}}^{+}\right) \hat{\partial}_{m}+\hat{\theta}^{\underline{\alpha}+} \partial_{\underline{\alpha-}}-z^{++} \partial_{\mathrm{u}}^{0} \\
& -\left(z^{++}\right)^{2} \frac{\partial}{\partial z^{++}}+z^{++} z^{0} \frac{\partial}{\partial z^{0}}+\left(2 z^{++} z^{--}-1\right) \frac{\partial}{\partial z^{--}}, \\
& D_{\mathrm{v}}^{--}=\frac{1}{\left(z^{0}\right)^{2}}\left(\partial_{\mathrm{u}}^{--}-2 i\left(\hat{\theta}^{-} \sigma^{m} \hat{\bar{\theta}}^{-}\right) \hat{\partial}_{m}+\hat{\theta}^{\underline{\alpha}-} \partial_{\underline{\alpha}+}-\frac{\partial}{\partial z^{++}}+z^{--} z^{0} \frac{\partial}{\partial z^{0}}+\left(z^{--}\right)^{2} \frac{\partial}{\partial z^{--}}\right) \text {, } \\
& D_{\mathrm{w}}^{0}=\partial_{\mathrm{u}}^{0}+\hat{\theta}^{\underline{\alpha}+} \hat{\partial}_{\underline{\alpha}+}-\hat{\theta}^{\underline{\alpha}-} \hat{\partial}_{\underline{\alpha}-}+2 z^{++} \partial_{z^{++}}-2 z^{--} \partial_{z^{--}}-z^{0} \frac{\partial}{\partial z^{0}} .
\end{aligned}
$$

Any twisted biholomorphic superfield $\mathcal{F}^{(n, m)}$ corresponds to a complex harmonic superfield $\mathcal{F}^{(n+m)}$ via $\mathcal{F}^{(n, m)}=\left(z^{0}\right)^{n} \mathcal{F}^{(n+m)}\left(\hat{x}, \hat{\theta}^{+}, u^{ \pm}\right)$, which transforms as

$$
\begin{aligned}
\delta \mathcal{F}^{(n+m)} & =\lambda^{m} \partial_{m} \mathcal{F}^{(n+m)}+\lambda^{\underline{\alpha}+} \partial_{\underline{\alpha}+} \mathcal{F}^{(n+m)}-\lambda_{\mathrm{u}}^{++} \partial_{\mathrm{u}}^{--} \mathcal{F}^{(n+m)}+n \Lambda \mathcal{F}^{(n+m)}, \\
\lambda^{m} & =\xi^{m}-2 i\left(\xi^{i} \sigma^{m} \hat{\bar{\theta}}^{-}\right) u_{i}^{+}-2 i\left(\hat{\theta}^{-} \sigma^{m} \bar{\xi}^{i}\right) u_{i}^{+}+2 i \lambda_{\mathrm{u}}^{++}\left(\hat{\theta}^{-} \sigma^{m} \hat{\bar{\theta}}^{-}\right) \\
\lambda^{\underline{\alpha}} & =\xi^{\underline{\alpha}} u_{i}^{+}-\lambda_{\mathrm{u}}^{++} \hat{\theta}^{\underline{\alpha}-} .
\end{aligned}
$$

The parameters $\lambda^{m}, \lambda^{\underline{\alpha}}, \lambda_{\mathrm{u}}^{++}$, and $\Lambda$ are independent of $\theta^{\underline{\alpha}-}, z^{++}, z^{--}$and $z^{0}$. They are interpreted as arising from the analytic general coordinate transformation

$$
\delta^{*} u^{i+}=\lambda_{\mathrm{u}}^{++} u^{i-}, \quad \delta^{*} u_{i}^{-}=0, \quad \delta^{*} \hat{x}^{m}=-\lambda^{m}, \quad \delta^{*} \hat{\theta}^{\underline{\alpha}+}=-\lambda^{\underline{\alpha}+},
$$

on the analytic space $\left(\hat{x}, \hat{\theta}^{+}, u^{ \pm}\right)$, with $\Lambda$ given by half of the infinitesimal Berezinian, $\Lambda=$ $\frac{1}{2}\left(\partial_{m} \lambda^{m}-\partial_{\underline{\alpha}+} \lambda^{\underline{\alpha}+}-\partial_{\mathrm{u}}^{--} \lambda_{\mathrm{u}}^{++}\right)$, equivalent to (5.6). Any analytic superfield transforming as (5.11) is called a primary analytic scalar of weight $n$. From the above results, one may show that the action

$$
S=\frac{i}{2 \pi} \int_{\mathcal{S}} \mathcal{U}^{++} \wedge \mathcal{U}^{--} \int \mathrm{d}^{4} \hat{x} \mathrm{~d}^{4} \hat{\theta}^{+} \mathscr{L}^{+4}
$$

is a superconformal invariant provided $\mathscr{L}^{+4}$ is an analytic scalar of weight two. This is just the rigid formulation of conventional harmonic superspace on the complexified $S^{2}$, and reproduces the rigid limit of the analytic superspace action principle (4.5) after taking

$$
\mathscr{L}^{(2,2)}=\left(z^{0}\right)^{2} \mathscr{L}^{+4}, \quad \mathcal{E}^{(-2,-2)}=\left(z^{0}\right)^{-2}\left(\mathcal{U}_{\zeta}^{++} \mathcal{U}_{\tilde{\zeta}}^{--}-\mathcal{U}_{\tilde{\zeta}}^{++} \mathcal{U}_{\tilde{\zeta}}^{--}\right)
$$




\subsection{The analytic basis in complex harmonic superspace}

In order to construct the curved analogue of (5.13), we must introduce the analytic basis in a curved geometry. We assume that this analytic basis (or analytic gauge) is accomplished using a twisted biholomorphic gauge transformation with gauge parameters that are homogeneous of degree zero in $v^{i}$ and $\bar{w}_{i}$ separately. Schematically, the analytic gauge arises as $\hat{\nabla}_{\mathcal{A}}=e^{\mathcal{B}} \nabla_{\mathcal{A}} e^{-\mathcal{B}}$ where $\nabla_{\mathcal{A}}$ is the covariant derivative in central gauge and $e^{\mathcal{B}}$ is a bridge operator. For the coordinates themselves, the bridge can be represented as a twisted biholomorphic coordinate transformation, taking the central basis $z^{\mathcal{M}}$ to analytic basis $\hat{z}^{\mathcal{M}}$ given by the generalization of (5.7),

$$
\begin{array}{ll}
\hat{x}^{m}=x^{m}+b^{m}, & \hat{\theta}^{\underline{\mu}}+=\frac{\theta^{\underline{\mu}} v_{i}^{+}}{\left(v^{+}, w^{-}\right)}+b^{\underline{\mu}+}, \quad \hat{\theta}^{\underline{\mu}}-\theta^{-} \underline{\underline{\mu}}^{-} w_{i}^{-}+b^{\underline{\mu}}- \\
\hat{v}^{i \pm}=v^{j \pm} b_{j}{ }^{i}, & \hat{w}^{i \pm}=w^{i \pm},
\end{array}
$$

in terms of twisted biholomorphic bridges $b$ that depend only on the harmonics $v^{i+}$ and $w_{i}^{-}$. These bridges carry vanishing $\mathrm{SU}(2)_{\mathrm{v}}$ charge and their $\mathrm{SU}(2)_{\mathrm{w}}$ charge is indicated; in addition, $b_{j}{ }^{i}$ must have unit determinant. Because $\nabla_{\underline{\alpha}}^{+}$annihilates $w^{i \pm}$ in the central gauge (i.e. there is no $\mathrm{SU}(2)_{\mathrm{w}}$ connection), it is evident that no bridge needs to be introduced for those harmonics. However, the presence of the $\mathrm{SU}(2)_{\mathrm{v}}$ connection in the central basis requires the bridges $b_{j}{ }^{i}$ to be nonzero, because in the central basis $\nabla_{A} v^{i+}=\mathcal{V}_{A}{ }_{j}{ }_{j} v^{j+} \neq 0$.

As a consequence of the twisted biholomorphy of the bridges, one can see that a number of harmonic derivatives take simple forms in the analytic basis:

$$
\hat{\nabla}_{\mathrm{w}}^{0}=\hat{\partial}_{\mathrm{w}}^{0}+\hat{\theta}^{\underline{\mu}} \hat{\partial}_{\underline{\mu}+}-\hat{\theta}^{\underline{\mu}} \hat{\partial}_{\underline{\mu}}, \quad, \quad \hat{\nabla}_{\mathrm{w}}^{--}=\hat{\partial}_{\mathrm{w}}^{--}, \quad \hat{\nabla}_{\mathrm{v}}^{0}=\hat{\partial}_{\mathrm{v}}^{0}, \quad \hat{\nabla}_{\mathrm{v}}^{++}=\hat{\partial}_{\mathrm{v}}^{++} .
$$

In addition, there is no obstruction to choosing $\hat{\nabla}_{\underline{\alpha}}^{+}$to simply be given by

$$
\hat{\nabla}_{\underline{\alpha}}^{+}=\left(\hat{v}^{+}, w^{-}\right) \delta_{\underline{\alpha}}^{\underline{\mu}} \frac{\partial}{\partial \hat{\theta}^{\underline{\mu}}-} .
$$

This involves not just a choice of analytic coordinates but also a choice of all the other gauges as well, both to trivialize the vielbein terms and to eliminate the connections; this is possible as a consequence of (3.13). ${ }^{10}$ The expression for $\hat{\nabla}_{\mathrm{w}}^{++}$is more elaborate:

$$
\begin{aligned}
\hat{\nabla}_{\mathrm{w}}^{++}= & \hat{\partial}_{\mathrm{w}}^{++}-H^{(2,2)} \hat{\partial}_{\mathrm{v}}^{--}+H^{(0,2)} \hat{\partial}_{\mathrm{v}}^{0}+H^{(-2,2)} \hat{\partial}_{\mathrm{v}}^{++}+H^{(0,2) m} \hat{\partial}_{m}+H^{(0,3) \underline{\mu}} \hat{\partial}_{\underline{\mu}}+H^{(0,1) \underline{\mu}} \hat{\partial}_{\underline{\mu}-} \\
& -\frac{1}{2} \Omega_{\mathrm{w}}^{++a b} M_{b a}-A_{\mathrm{w}}^{++} \mathbb{A}-B_{\mathrm{w}}^{++} \mathbb{D}-F^{(0,2) a} K_{a}-F^{(1,2) \underline{\alpha}} S_{\underline{\alpha}}^{-}+F^{(-1,2) \underline{\alpha}} S_{\underline{\alpha}}^{+} . \quad \text { (5.18) }
\end{aligned}
$$

The contributions to the vielbein, denoted by $H$, coincide with the similarly-named objects in the conventional harmonic superspace approach [52] (and which were introduced earlier in [37]) up to redefinitions to be discussed in a moment.

A key observation one should make about (5.18) is the absence of additional contributions involving $\hat{\partial}_{\mathrm{w}}^{0}$ and $\hat{\partial}_{\mathrm{w}}^{--}$. This is a consequence of the simple form for the analytic basis

\footnotetext{
${ }^{10}$ We took in $[10]$ the analytic gauge $\nabla_{\underline{\alpha}}^{+}=\partial_{\underline{\alpha}}^{+}$, choosing the $\theta^{+}$coordinates to carry $\mathrm{U}(1)_{\mathrm{v}}$ charge. This choice is also possible here at the cost of breaking with standard harmonic superspace conventions.
} 
for the $\mathrm{SU}(2)_{\mathrm{w}}$ harmonics. However, there is a complication hidden within (5.18): not all of the connections are twisted biholomorphic. In particular,

$$
\begin{aligned}
& \hat{\partial}_{\mathrm{v}}^{++} F^{(-1,2) \underline{\alpha}}=F^{(1,2) \underline{\alpha}}, \quad \hat{\partial}_{\mathrm{v}}^{++} F^{(1,2) \underline{\alpha}}=0, \\
& \hat{\partial}_{\mathrm{v}}^{++} H^{(-2,2)}=2 H^{(0,2)}, \quad \hat{\partial}_{\mathrm{v}}^{++} H^{(0,2)}=H^{(2,2)}, \quad \hat{\partial}_{\mathrm{v}}^{++} H^{(2,2)}=0,
\end{aligned}
$$

while the vielbeins $H^{(0,3) \underline{\mu}}$ and $H^{(0,1) \underline{\mu}}$ obey

$$
\hat{\partial}_{\mathrm{w}}^{--} H^{(0,3) \underline{\mu}}=-\hat{\theta}^{\underline{\mu}+}, \quad \hat{\partial}_{\mathrm{w}}^{--} H^{(0,1) \underline{\mu}}=-\hat{\theta}^{\underline{\mu}-} .
$$

To make these features explicit, it will be useful to adopt a change of coordinates to the complex variables $u^{i \pm}, z^{ \pm \pm}$, and $z^{0}$ defined in section 2.3. Analogous definitions in the analytic basis of $\hat{u}^{i \pm}, \hat{z}^{ \pm \pm}$, and $\hat{z}^{0}$ in terms of $\hat{v}^{i \pm}$ and $\hat{w}^{i \pm}$ leads to

$$
\begin{aligned}
& \hat{z}^{++}=z^{++}+b^{++}, \quad \hat{z}^{0}=z^{0} b^{0}, \quad \hat{z}^{--}=\left(b^{0}\right)^{2}\left(z^{--}+b^{--}\right), \\
& \hat{u}^{i+}=u^{i+}-b^{++} u^{i-}, \quad \hat{u}_{i}^{-}=u_{i}^{-} \text {. }
\end{aligned}
$$

The three bridges $b^{ \pm \pm}$and $b^{0}$ are nonlinearly related to the $\mathrm{SU}(2)_{\mathrm{v}}$ bridge $b_{i}{ }^{j}$. The bridges $b^{++}, b^{m}, b^{\underline{\mu}}+$ and $b^{\underline{\mu}-}$ appeared in the harmonic superspace context in [52]; the bridges $b^{0}$ and $b^{--}$did not appear there because the additional complex coordinates $\hat{z}^{0}$ and $\hat{z}^{--}$are not needed to describe analytic superfields. ${ }^{11}$ In terms of these coordinates, we now have

$$
\begin{aligned}
\hat{\nabla}_{\mathrm{w}}^{0} & =\hat{\partial}_{\mathrm{u}}^{0}+2 \hat{z}^{++} \partial_{\hat{z}^{++}}-2 \hat{z}^{--} \hat{\partial}_{z^{--}}-\hat{z}^{0} \hat{\partial}_{z^{0}}+\hat{\theta}^{\mu+} \hat{\partial}_{\underline{\mu}+}-\hat{\theta}^{\underline{\mu}} \hat{\partial}_{\underline{\mu}-}, \\
\hat{\nabla}_{\mathrm{w}}^{--} & =\frac{\partial}{\partial \hat{z}^{++}}, \quad \hat{\nabla}_{\mathrm{v}}^{++}=\left(\hat{z}^{0}\right)^{2} \frac{\partial}{\partial \hat{z}^{--}}, \quad \hat{\nabla}_{\mathrm{v}}^{0}=\hat{z}^{0} \frac{\partial}{\partial \hat{z}^{0}}, \\
\hat{\nabla}_{\underline{\alpha}}^{+} & =\hat{z}^{0} \delta_{\underline{\alpha}^{\underline{\mu}}} \frac{\partial}{\partial \hat{\theta}^{\underline{\mu}}} .
\end{aligned}
$$

To simplify the conditions (5.19) on the connections for $\nabla_{\mathrm{w}}^{++}$, it will be useful to introduce new vielbeins $\mathcal{H}$ that are independent of $\hat{z}^{ \pm \pm}$and $\hat{z}^{0}$ :

$$
\begin{aligned}
H^{m(0,2)} & \equiv \mathcal{H}^{++m}, \quad H^{(0,3) \underline{\mu}} \equiv \mathcal{H}^{+++\underline{\mu}}-\hat{z}^{++} \hat{\theta}^{\underline{\mu}}, \quad H^{(0,1) \underline{\mu}} \equiv \mathcal{H}^{+\underline{\mu}}-\hat{z}^{++} \hat{\theta}^{\underline{\mu}-}, \\
\frac{H^{(2,2)}}{\left(\hat{z}^{0}\right)^{2}} & \equiv \mathcal{H}^{+4}, \quad H^{(0,2)} \equiv \mathcal{H}^{++}+\hat{z}^{--} \mathcal{H}^{+4} \\
\left(\hat{z}^{0}\right)^{2} H^{(-2,2)} & \equiv \mathcal{H}^{0}+2 \hat{z}^{--} \mathcal{H}^{++}+\left(\hat{z}^{--}\right)^{2} \mathcal{H}^{+4}
\end{aligned}
$$

It is convenient to similarly modify the $S$-supersymmetry connections as well:

$$
F^{(1,2) \underline{\alpha}} \equiv \hat{z}^{0} \mathcal{F}^{+++\underline{\alpha}}, \quad F^{(-1,2) \underline{\alpha}} \equiv \frac{1}{\hat{z}^{0}}\left(\mathcal{F}^{+\underline{\alpha}}+\hat{z}^{--} \mathcal{F}^{+++\underline{\alpha}}\right) .
$$

Then (5.18) may be rewritten

$$
\hat{\nabla}_{\mathrm{w}}^{++}=\hat{\partial}_{\mathrm{u}}^{++}-\hat{z}^{++} \hat{\nabla}_{\mathrm{w}}^{0}+\left(\hat{z}^{++}\right)^{2} \hat{\nabla}_{\mathrm{w}}^{--}+\mathcal{H}^{+4}\left(\hat{\partial}_{z^{++}}-\hat{\partial}_{\mathrm{u}}^{--}\right)+\mathcal{H}^{++}\left(\hat{\nabla}_{\mathrm{v}}^{0}+2 \hat{z}^{--} \hat{\partial}_{z^{--}}\right)
$$

\footnotetext{
${ }^{11}$ These bridges match those given in [38] for the complex harmonic description of quaternionic sigma models. This is a natural consequence of the biholomorphic analyticity assumed there, which we follow.
} 


$$
\begin{aligned}
& +\left(\mathcal{H}^{0}-1\right) \hat{\partial}_{z^{--}}+\mathcal{H}^{++m} \hat{\partial}_{m}+\mathcal{H}^{+++} \underline{\mu} \hat{\partial}_{\underline{\mu}+}+\mathcal{H}^{+\underline{\mu}} \hat{\partial}_{\underline{\mu}-}-\frac{1}{2} \Omega_{\mathrm{w}}^{++a b} M_{b a} \\
& -A_{\mathrm{w}}^{++} \mathbb{A}-B_{\mathrm{w}}^{++} \mathbb{D}-F_{\mathrm{w}}^{++a} K_{a}+\mathcal{F}^{+\underline{\alpha}} S_{\underline{\alpha}}^{+}-\mathcal{F}^{+++\underline{\alpha}}\left(\hat{z}^{0} S_{\underline{\alpha}}^{-}-\frac{\hat{z}^{--}}{\hat{z}^{0}} S_{\underline{\alpha}}^{+}\right) .
\end{aligned}
$$

Each of the connection superfields above is twisted biholomorphic with vanishing $U(1)_{v}$ charge, depending only on the complex harmonics. Up to sign conventions, the superfields $\mathcal{H}^{+4}, \mathcal{H}^{+++} \underline{\mu}, \mathcal{H}^{++m}$, and $\mathcal{H}^{+} \underline{\mu}$ coincide with similarly named objects in the conventional harmonic superspace description of conformal supergravity [37, 52]. The importance of the connections $B_{\mathrm{w}}^{++}$and $\mathcal{H}^{++}$will be addressed in the next subsection. The absence of the remaining connections in [52] is apparent when one recalls that $\hat{\nabla}_{\mathrm{w}}^{++}$always acts on analytic twisted biholomorphic primary scalars, and so these connections tend to drop out.

At this stage, we could proceed further and analyze the full structure of the analytic basis, including the connections within $\hat{\nabla}_{\mathrm{v}}^{--}$. However, this will not be directly necessary: our main motivation is to provide a covariant description, and a full construction of the analytic basis connections is not necessary for that purpose. However, it may be useful to note the consequences of $\left[\hat{\nabla}_{\mathrm{w}}^{++}, \hat{\nabla}_{\underline{\alpha}}^{+}\right]=0$. One finds (using $\partial_{\underline{\alpha}}^{+} \equiv \partial_{\underline{\alpha}-}$ ) that several of the vielbeins and the combination $\mathcal{H}^{++}-B_{\mathrm{w}}^{++}$are analytic,

$$
\partial_{\underline{\alpha}}^{+} \mathcal{H}^{+4}=\partial_{\underline{\alpha}}^{+} \mathcal{H}^{++m}=\partial_{\underline{\alpha}}^{+} \mathcal{H}^{+++\underline{\mu}}=\partial_{\underline{\alpha}}^{+}\left(\mathcal{H}^{++}-B_{\mathrm{w}}^{++}\right)=0,
$$

while the other vielbeins are less strongly constrained,

$$
\partial_{\alpha}^{+} \mathcal{H}^{+\dot{\mu}}=0, \quad\left(\partial^{+}\right)^{2} \mathcal{H}^{0}=\left(\bar{\partial}^{+}\right)^{2} \mathcal{H}^{0}=0 .
$$

The other connections are entirely determined in terms of the vielbeins as

$$
\begin{array}{rlrl}
B_{\mathrm{w}}^{++} & =2 \mathcal{H}^{++}-\frac{1}{2}\left(\partial_{\alpha}^{+} \mathcal{H}^{\alpha+}+\bar{\partial}_{\dot{\alpha}}^{+} \mathcal{H}^{\dot{\alpha}+}\right), \Omega_{\mathrm{w}}^{++}{ }_{\alpha \beta} & =-\partial_{(\alpha}^{+} \mathcal{H}_{\beta)}^{+}, \\
A_{\mathrm{w}}^{++} & =-\frac{i}{4}\left(\partial_{\alpha}^{+} \mathcal{H}^{\alpha+}-\bar{\partial}_{\dot{\alpha}}^{+} \mathcal{H}^{\dot{\alpha}+}\right), & F_{\mathrm{w}}^{++}{ }_{\alpha \dot{\beta}} & =-\frac{i}{4} \partial_{\alpha}^{+} \bar{\partial}_{\dot{\beta}}^{+} \mathcal{H}^{0}, \\
\mathcal{F}^{+++\alpha} & =-\frac{1}{8}\left(\partial^{+}\right)^{2} \mathcal{H}^{\alpha+}, & \mathcal{F}^{+\alpha} & =-\frac{1}{4} \partial^{\alpha+} \mathcal{H}^{0} .
\end{array}
$$

The conditions (5.27) and (5.28) are a consequence of the strong gauge choice (5.17) made for $\nabla_{\alpha}^{+}$. These can be simplified still further by imposing the additional gauges $\mathcal{H}^{++}=$ $\mathcal{H}^{0}=0$ and $\mathcal{H}^{\underline{\mu}}{ }^{+}=\hat{\theta}^{\underline{\mu}}{ }^{+}$, which imply that $\hat{z}^{0}, \hat{z}^{--}$, and $\hat{\theta}^{\underline{\mu}}{ }^{-}$are respectively chosen to solve $\nabla_{\mathrm{w}}^{++} \log \hat{z}^{0}=\hat{z}^{++}, \nabla_{\mathrm{w}}^{++}\left(\hat{z}^{--} /\left(\hat{z}^{0}\right)^{2}\right)=-1 /\left(\hat{z}^{0}\right)^{2}$, and $\nabla_{\mathrm{w}}^{++}\left(\hat{\theta}^{\underline{\mu}}-/ \hat{z}^{0}\right)=\hat{\theta}^{\underline{\mu}}+/ \hat{z}^{0}$.

\subsection{Curved harmonic superspace and analytic densities}

In the conventional formulation of curved harmonic superspace [52], one remains in the analytic gauge while generalizing the transformations (5.12) and the action (5.13). That is, on the analytic superspace, the coordinates $\hat{x}, \hat{\theta}^{+}$, and $\hat{u}^{ \pm}$transform as

$$
\delta^{*} \hat{u}^{i+}=\hat{\lambda}^{++} \hat{u}^{i-}, \quad \delta^{*} \hat{u}^{i-}=0, \quad \delta^{*} \hat{x}^{m}=-\hat{\lambda}^{m}, \quad \delta^{*} \hat{\theta}^{\underline{\alpha}+}=-\hat{\lambda}^{\underline{\alpha}+},
$$

involving unconstrained analytic parameters $\hat{\lambda}$. The action

$$
S=\frac{i}{2 \pi} \int_{\mathcal{S}} \hat{\mathcal{U}}^{++} \wedge \hat{\mathcal{U}}^{--} \int \mathrm{d}^{4} \hat{x} \mathrm{~d}^{4} \hat{\theta}^{+} \hat{\mathscr{L}}^{+4}
$$


is invariant provided $\hat{\mathscr{L}}^{+4}$ transforms as a primary analytic scalar of weight two,

$$
\delta \hat{\mathscr{L}}^{+4}=\left(\hat{\lambda}^{m} \hat{\partial}_{m}+\hat{\lambda}^{\underline{\alpha}+} \hat{\partial}_{\underline{\alpha}+}-\hat{\lambda}^{++} \hat{\partial}_{\mathrm{u}}^{--}\right) \hat{\mathscr{L}}^{+4}+2 \hat{\Lambda} \hat{\mathscr{L}}^{+4},
$$

where $2 \hat{\Lambda}:=\hat{\partial}_{m} \hat{\lambda}^{m}-\hat{\partial}_{\underline{\alpha}+} \hat{\lambda}^{\underline{\alpha}+}-\hat{\partial}_{\mathrm{u}}^{--} \hat{\lambda}^{++}$.

To establish the action above, it helps to slightly modify our formulation (4.5) for the action in a general gauge. Instead of the measure factor $\mathrm{d}^{2} \zeta$ built out of the coordinates $\zeta$ and $\tilde{\zeta}$ parametrizing $\mathcal{S}$ directly, we wish to use the complex harmonic measure $\mathcal{U}^{++} \wedge \mathcal{U}^{--}$, with the complex harmonics $u^{ \pm}$implicitly depending on $\zeta$ and $\tilde{\zeta}$. So we first reexpand the vielbein one-forms using the complex harmonic coordinates:

$$
\begin{aligned}
E^{\mathcal{A}}= & \mathrm{d} z^{M} E_{M}{ }^{\mathcal{A}}+\mathcal{U}^{++} E_{\mathrm{u}++}{ }^{\mathcal{A}}+\mathcal{U}^{0} E_{\mathrm{u} 0}{ }^{\mathcal{A}}+\mathcal{U}^{--} E_{\mathrm{u}--} \mathcal{A} \\
& +\mathrm{d} z^{++} E_{z^{++}}{ }^{\mathcal{A}}+\mathrm{d} z^{--} E_{z^{--}}{ }^{\mathcal{A}}+\mathrm{d} z^{0} E_{z^{0}}{ }^{\mathcal{A}} .
\end{aligned}
$$

The one-forms $\mathcal{U}^{++}, \mathcal{U}^{--}$and $\mathcal{U}^{0}$ constitute a fixed anholonomic frame. Then the analytic superspace action (4.5) becomes

$$
\begin{aligned}
& \frac{i}{2 \pi} \int_{\mathcal{S}} \mathcal{U}^{++} \wedge \mathcal{U}^{--} \int \mathrm{d}^{4} x \mathrm{~d}^{4} \theta^{+} \mathcal{E}^{(-2,-2)} \mathscr{L}^{(2,2)}, \\
& \mathcal{E}^{(-2,-2)}=\operatorname{sdet}\left(\begin{array}{cccc}
E_{m}{ }^{a} & E_{m} \underline{\alpha}+ & E_{m}{ }^{\mathrm{v}++} & E_{m}{ }^{\mathrm{w}--} \\
E_{\underline{\mu}+}{ }^{a} & E_{\underline{\mu}+} \underline{\alpha}+ & E_{\underline{\mu}+}{ }^{\mathrm{v}++} & E_{\underline{\mu}+}{ }^{\mathrm{w}--} \\
E_{\mathrm{u}++}{ }^{a} & E_{\mathrm{u}++} \underline{\underline{\alpha}}+ & E_{\mathrm{u}++}{ }^{\mathrm{v}++} & E_{\mathrm{u}++}{ }^{\mathrm{w}--} \\
E_{\mathrm{u}--}{ }^{a} & E_{\mathrm{u}--}{ }^{\underline{\alpha}+} & E_{\mathrm{u}---}{ }^{\mathrm{v}++} & E_{\mathrm{u}--}{ }^{\mathrm{w}--}
\end{array}\right) .
\end{aligned}
$$

Under a diffeomorphism, one still finds $\delta E_{\mathcal{M}^{\mathcal{A}}}=\xi^{\mathcal{N}} \partial_{\mathcal{N}} E_{\mathcal{M}^{\mathcal{A}}}+\partial_{\mathcal{M}} \xi^{\mathcal{N}} E_{\mathcal{N}^{\mathcal{A}}}$ but must interpret $\partial_{\mathcal{M}}$ as including the non-commuting derivatives $\partial_{\mathrm{u}}^{ \pm \pm}$and $\partial_{\mathrm{u}}^{0}$. In particular, the measure $\mathcal{E}^{(-2,-2)}$ transforms under diffeomorphisms of the coordinates $x, \theta^{+}, u_{i}^{ \pm}$as

$$
\delta \mathcal{E}^{(-2,-2)}=(-)^{\mathbf{m}} \partial_{\mathbf{m}}\left(\xi^{\mathbf{m}} \mathcal{E}^{(-2,-2)}\right), \quad \mathbf{m}=(m, \underline{\mu}+, \mathrm{u}++, \mathrm{u}--)
$$

Now let us go to the analytic basis. Using the results in appendix A, one can show that within the analytic gauge, $\hat{\mathcal{E}}^{(-2,-2)}$ is independent of $\hat{\theta}^{\underline{\mu}}$ and $\hat{z}^{ \pm \pm}$,

$$
\hat{\nabla}_{\underline{\alpha}}^{+} \hat{\mathcal{E}}^{(-2,-2)}=\hat{\nabla}_{\mathrm{v}}^{++} \hat{\mathcal{E}}^{(-2,-2)}=\hat{\nabla}_{\mathrm{w}}^{--} \hat{\mathcal{E}}^{(-2,-2)}=0
$$

and possesses charge $(-2,+2)$ under the action of the $\mathrm{U}(1)_{\mathrm{v}} \times \mathrm{U}(1)_{\mathrm{w}}$ derivatives:

$$
\hat{\nabla}_{\mathrm{v}}^{0} \hat{\mathcal{E}}^{(-2,-2)}=-2 \hat{\mathcal{E}}^{(-2,-2)}, \quad \hat{\nabla}_{\mathrm{w}}^{0} \hat{\mathcal{E}}^{(-2,-2)}=+2 \hat{\mathcal{E}}^{(-2,-2)} .
$$

Note that these differential conditions are distinct from the transformation properties of the measure under covariant diffeomorphisms. The above conditions hold only in the analytic gauge and arise as a consequence of the explicit way we have chosen the Grassmann coordinates. Now combine the scalar Lagrangian with the measure to give the Lagrangian density with (analytic) charge $(0,+4)$,

$$
\hat{\mathscr{L}}^{+4}=\hat{\mathcal{E}}^{(-2,-2)} \mathscr{L}^{(2,2)} . \quad \hat{\nabla}_{\mathrm{v}}^{0} \hat{\mathcal{L}}^{+4}=0, \quad \hat{\nabla}_{\mathrm{w}}^{0} \hat{\mathcal{L}}^{+4}=+4 \hat{\mathcal{L}}^{+4} .
$$

It transforms under analytic coordinate transformations precisely as in (5.31). For the invariant action, we recover (5.30). 


\subsection{Examples in the analytic basis}

Let us verify agreement with [52] by constructing two simple actions in the analytic basis.

The $Q^{+}$hypermultiplet action. We start with the general form of the $Q^{\text {at }}$ hypermultiplet action

$$
\mathscr{L}^{(2,2)}=\frac{1}{2} L_{\mathrm{a}}^{+} \nabla_{\mathrm{w}}^{++} Q^{\mathrm{a}+}+H^{(2,2)} .
$$

$Q^{\mathrm{a}+}$ is a scalar multiplet of weight 1 , while $L_{\mathrm{a}}^{+}$and $H^{(2,2)}$ are functions of the appropriate homogeneities for their weights. Let us denote $\hat{\mathcal{E}} \equiv \hat{\mathcal{E}}^{(-2,-2)}$ in this section to keep notation relatively simple. Then we make the change of variables to the scalar density $\widehat{Q}^{\mathrm{a}+}=$ $(\hat{\mathcal{E}})^{1 / 2} Q^{\mathrm{a}+}$. Using the conformal properties of the fields, we find

$$
L_{\mathrm{a}}^{+}\left(Q, w^{-}\right)=(\hat{\mathcal{E}})^{-1 / 2} L_{\mathrm{a}}^{+}\left(\widehat{Q}^{+}, w^{-}\right), \quad H^{(2,2)}=(\hat{\mathcal{E}})^{-1} H^{+4}\left(\widehat{Q}^{+}, w^{-}\right) .
$$

Then the analytic Lagrangian $\hat{\mathscr{L}}^{+4} \equiv \hat{\mathcal{E}} \mathscr{L}^{(2,2)}$ can be written

$$
\begin{aligned}
& \hat{\mathscr{L}}^{+4}=\frac{1}{2} L_{\mathrm{a}}^{+} \hat{\mathcal{E}}^{1 / 2}\left(\hat{\mathcal{D}}^{++}+\left(\mathcal{H}^{++}-B_{\mathrm{w}}^{++}\right)\right) \frac{\widehat{Q}^{\mathrm{a}+}}{\hat{\mathcal{E}}^{1 / 2}}+H^{+4}, \\
& \hat{\mathcal{D}}^{++} \equiv \hat{\partial}_{\mathrm{u}}^{++}-\mathcal{H}^{+4} \hat{\partial}_{\mathrm{u}}^{--}+\mathcal{H}^{++m} \hat{\partial}_{m}+\mathcal{H}^{+++\mu} \hat{\partial}_{\mu+} .
\end{aligned}
$$

To proceed further, we require the following identity in the analytic basis:

$$
\hat{\mathcal{D}}^{++} \hat{\mathcal{E}}-2\left(\mathcal{H}^{++}-B_{\mathrm{w}}^{++}\right) \hat{\mathcal{E}}=\left(\hat{\partial}_{\mathrm{u}}^{--} \mathcal{H}^{+4}-\hat{\partial}_{m} \mathcal{H}^{++m}+\hat{\partial}_{\mu+} \mathcal{H}^{+++\mu}\right) \hat{\mathcal{E}} \equiv-\Gamma^{++} \hat{\mathcal{E}} .
$$

Its proof follows by noting that the harmonic superspace integral of

$$
\hat{\mathcal{E}} \hat{\nabla}_{\mathrm{w}}^{++} \mathcal{F}^{(2,0)}=\hat{\mathcal{E}}\left(\hat{\mathcal{D}}^{++} \mathcal{F}^{(2,0)}+2\left(\mathcal{H}^{++}-B_{\mathrm{w}}^{++}\right) \mathcal{F}^{(2,0)}\right)
$$

is a total derivative for any $\mathcal{F}^{(2,0)}$. Then we recover

$$
\hat{\mathscr{L}}^{+4}=\frac{1}{2} L_{\mathrm{a}}^{+}\left(\hat{\mathcal{D}}^{++}+\frac{1}{2} \Gamma^{++}\right) \widehat{Q}^{\mathrm{a}+}+H^{+4} .
$$

This is the correct analytic basis action, expressed in terms of scalar densities $\widehat{Q}^{\mathrm{a}+}$. As anticipated, the connection $\left(\mathcal{H}^{++}-B_{\mathrm{w}}^{++}\right)$has been replaced by the analytic quantity $\frac{1}{2} \Gamma^{++}$.

The improved tensor multiplet action. As a similar exercise, we now show how to recover the improved tensor multiplet action in the analytic basis. Beginning with (4.30), we must make the change of variables $\widehat{Q}_{0}^{i+}=(\hat{\mathcal{E}})^{1 / 2} Q_{0}^{i+}$ and $\widehat{L}^{++}=\hat{\mathcal{E}} L^{++}$. Then the analytic Lagrangian density becomes

$$
\hat{\mathscr{L}}^{+4}=\frac{1}{2} \frac{\left(\widehat{g}^{++}\right)^{2}}{\left(\widehat{\Omega}_{0}\right)^{2}}+\frac{1}{2} \widehat{Q}_{0 i}^{+} \hat{\mathcal{D}}^{++} \widehat{Q}_{0}^{i+}-2 \frac{\widehat{g}^{++} c_{i j} w^{i-}}{\widehat{\Omega}_{0}}\left(\hat{\mathcal{D}}^{++}+\frac{1}{2} \Gamma^{++}\right) \widehat{Q}_{0}^{j+} .
$$

We know that $\widehat{Q}_{0}^{i+}$ may be any analytic function: in the analytic gauge, we can simply choose it to be $\hat{u}^{i+}$. Doing so, it is not hard to show

$$
\hat{\mathscr{L}}^{+4}=\frac{1}{2}\left(\widehat{g}^{++}\right)^{2}-\Gamma^{++} C^{-+} \widehat{g}^{++}+\frac{1}{2} \mathcal{H}^{+4}\left(1+4 \widehat{g}^{++} C^{--}\right) .
$$

This matches the action of [56] up to the redefinitions $C^{++} \rightarrow \frac{1}{2} C^{++}$and $L^{++} \rightarrow \frac{1}{2} L^{++}$, the difference in sign $\mathcal{H}^{+4} \rightarrow-\mathcal{H}^{+4}$, and an overall normalization. 


\subsection{Harmonic superspace on $\mathcal{M}^{4 \mid 8} \times S^{2}$}

Finally, let us address the relationship between the covariant formulation presented here and that of Galperin, Ky, and Sokatchev [26] (see also [25]). These papers established that within a superspace gauging only the Lorentz group and superdiffeomorphisms, the integrability conditions for analytic superfields coincided with the torsion constraints of the simplest version of $\mathcal{N}=2$ Einstein supergravity [50, 51]. Because of absence of $\mathrm{SU}(2)_{R}$ connections, one can introduce harmonics as external coordinates annihilated by the covariant spinor derivatives. The harmonics are identified simply with the external automorphism group $\mathrm{SU}(2)_{A}$, and so a single set of real harmonics and a real auxiliary $S^{2}$ is sufficient. Within this framework, the analytic prepotentials of Einstein supergravity were uncovered and explicit formulae for connections and vielbeins were worked out. However, certain features were not explored. A general component action analogous to (4.18) was not given, nor were the superspace actions cast in a manifestly covariant form. Although it is clear that such results could be constructed directly as we have done using conformal superspace, it is instructive to consider instead how to recover this Poincaré harmonic superspace framework directly from the conformal one.

Recall that this $\mathcal{N}=2$ Einstein supergravity corresponds to conformal supergravity coupled to two compensators: a vector multiplet $W$ and a non-linear multiplet $L_{\mathrm{a}}{ }^{i}$. Their lowest components fix the dilatation and the $\mathrm{SU}(2) \times \mathrm{U}(1) R$-symmetries and lead to an off-shell supergravity involving only gauged Lorentz transformations, diffeomorphisms, and supersymmetry. One of the lowest fermions fixes the $S$-supersymmetry gauge and the other becomes a dimension-1/2 matter field.

To describe the same supergravity at the superfield level, one can introduce superfields $W$ and $L_{\mathrm{a}}{ }^{i}$ in conformal superspace and adopt the dilatation- $\mathrm{U}(1)_{R}$ gauge $W=1$ and the $\mathrm{SU}(2)_{R}$ gauge $L_{\mathrm{a}}{ }^{i}=\delta_{\mathrm{a}}{ }^{i}$. Actually, it is instructive to construct the new superspace not by gauge-fixing explicitly but by using the superfields to redefine the vielbein and spin connection to compensate the symmetries that should be fixed. We may do this in two steps. First, using the superfield $W$, convert conformal superspace derivatives $\nabla_{A}$ to $\mathrm{SU}(2)$ superspace derivatives $\mathcal{D}_{A}$ (see [28] for the $\mathcal{N}=2$ case or [58] for a pedagogical discussion in $\mathcal{N}=1$ ). The $\mathcal{D}_{A}$ carry no dilatation weight or $\mathrm{U}(1)_{R}$ charge and are conformally inert. Provided one acts only on primary superfields whose Weyl and $\mathrm{U}(1)_{R}$ weights have been removed with the compensator, the dilatation, $\mathrm{U}(1)_{R}$ and special conformal connections become inert.

Next, using $\mathrm{SU}(2)$ superspace, we can repeat the construction of covariant harmonic superspace. The main details do not change; in particular, because the supergeometry includes the $\mathrm{SU}(2) R$-symmetry group, two sets of real harmonics are still necessary. The algebra of spinor covariant derivatives $\mathcal{D}_{\underline{\alpha}}^{+}$is [21]

$$
\left\{\mathcal{D}_{\alpha}^{+}, \mathcal{D}_{\beta}^{+}\right\}=2 S^{++} M_{\alpha \beta}+4 Y_{\alpha \beta} \mathcal{D}_{\mathrm{v}}^{++}, \quad\left\{\mathcal{D}_{\alpha}^{+}, \overline{\mathcal{D}}_{\dot{\beta}}^{+}\right\}=G_{\alpha \dot{\beta}} \mathcal{D}_{\mathrm{v}}^{++},
$$

consistent with the existence of twisted biholomorphic analytic scalars. Now we may introduce the second compensator and build $\mathrm{SU}(2)_{R}$-inert derivatives in the central basis:

$$
\widetilde{\mathcal{D}}_{\underline{\alpha} \mathrm{a}}=L_{\mathrm{a}}^{i}\left(\mathcal{D}_{\underline{\alpha} i}+\left(\mathcal{D}_{\underline{\alpha} i} L_{\mathrm{b}}{ }^{j}\right) L^{\mathrm{b}}{ }_{k} I^{k}{ }_{j}\right) \text {. }
$$


The second term modifies the $\mathrm{SU}(2)_{R}$ connection so that $L_{\mathrm{a}}{ }^{i}$ is covariantly constant. In the gauge where $L_{\mathrm{a}}{ }^{i}=\delta_{\mathrm{a}}{ }^{i}$, its effect is to eliminate the $\mathrm{SU}(2)_{R}$ connection entirely. An important check is to verify that the algebra of covariant derivatives still permits analytic multiplets. One finds that ${ }^{12}$

$$
\left\{\widetilde{\mathcal{D}}_{\alpha}^{(\mathrm{a}}, \widetilde{\mathcal{D}}_{\beta}^{\mathrm{b})}\right\}=\Psi_{(\alpha}^{(\mathrm{a}} \widetilde{\mathcal{D}}_{\beta)}^{\mathrm{b})}+\text { curvatures },
$$

where the fermion $\Psi_{\alpha}^{\mathrm{a}}$ is the dimension- $1 / 2$ fermion present in the non-linear compensator, now reinterpreted as a torsion superfield. We refer to the superspace associated with $\widetilde{\mathcal{D}}_{A}$ as "Einstein superspace". ${ }^{13}$

This shows how to arrive at the right supergeometry from the central basis, but what about from a more general gauge? Let the general gauge covariant derivatives in complex harmonic $\mathrm{SU}(2)$ superspace be denoted $\mathcal{D}_{\mathcal{A}}=\left(\mathcal{D}_{A}, \mathcal{D}_{\mathrm{v} a}, \mathcal{D}_{\mathrm{w} \bar{a}}\right)$. The non-linear multiplet superfields $L^{ \pm \pm}$and $L^{\mp \pm}$ carry both $\mathrm{U}(1)_{\mathrm{w}}$ and $\mathrm{U}(1)_{\mathrm{v}}$ charge (in the central basis these are $L^{\mathrm{a} i} w_{\mathrm{a}}^{ \pm} v_{i}^{ \pm}$and $\left.L^{\mathrm{a} i} w_{\mathrm{a}}^{\mp} v_{i}^{ \pm}\right)$. We introduce the compensated harmonic derivatives

$$
\begin{aligned}
\widetilde{\mathcal{D}}^{++} & :=\mathcal{D}_{\mathrm{w}}^{++}+\frac{1}{\left(L^{-+}\right)^{2}} \mathcal{D}_{\mathrm{v}}^{++}+N^{++} \mathcal{D}_{\mathrm{w}}^{0}-\left(N^{++}\right)^{2} \mathcal{D}_{\mathrm{w}}^{--}, \\
\widetilde{\mathcal{D}}^{0} & :=\mathcal{D}_{\mathrm{v}}^{0}+\mathcal{D}_{\mathrm{w}}^{0}+2 \frac{L^{--}}{L^{-+}} \mathcal{D}_{\mathrm{v}}^{++}-2 N^{++} \mathcal{D}_{\mathrm{w}}^{--}, \\
\widetilde{\mathcal{D}}^{--} & :=\left(L^{-+}\right)^{2} \mathcal{D}_{\mathrm{v}}^{--}+\mathcal{D}_{\mathrm{w}}^{--}-\left(L^{--}\right)^{2} \mathcal{D}_{\mathrm{v}}^{++}-L^{--} L^{-+} \mathcal{D}_{\mathrm{v}}^{0} .
\end{aligned}
$$

These act on twisted biholomorphic superfields, preserving their twisted biholomorphy. They also annihilate $L^{ \pm \pm}$and $L^{\mp \pm}$, and on the field dependent combinations $U^{\mathrm{a}+}:=\frac{L^{\mathrm{a}+}}{L^{-+}}$ and $U^{\mathrm{a}-}:=w^{\mathrm{a}-}$ the compensated harmonic derivatives formally act as if they were simple harmonic derivatives, i.e. $\widetilde{\mathcal{D}}^{++} U^{\mathrm{a}-}=U^{\mathrm{a}+}$, etc. Furthermore, because $L^{-+}$is covariantly constant and twisted biholomorphic, one may use it to trade $\mathrm{U}(1)_{\mathrm{v}}$ for $\mathrm{U}(1)_{\mathrm{w}}$ charge of any superfield. These observations are clear in the central gauge where $L_{\mathrm{a}}{ }^{i}=\delta_{\mathrm{a}}{ }^{i}$. There $L^{-+}$ is just $z^{0}, U^{\mathrm{a} \pm}=u^{\mathrm{a} \pm}$, and $\widetilde{\mathcal{D}}^{ \pm \pm}$and $\widetilde{\mathcal{D}}^{0}$ reduce to $\partial_{\mathrm{u}}^{ \pm \pm}$and $\partial_{\mathrm{u}}^{0}$.

The corresponding spinor derivatives in a general gauge are built from the $\mathrm{SU}(2)$ superspace derivatives as

$$
\begin{aligned}
& \widetilde{\mathcal{D}}_{\alpha}^{+}=\frac{1}{L^{-+}}\left(\mathcal{D}_{\alpha}^{+}+\left(\mathcal{D}_{\alpha}^{+} L_{\mathrm{b}}^{+}\right) L^{\mathrm{b}-} D_{\mathrm{v}}^{0}+\left(\mathcal{D}_{\alpha}^{+} L_{\mathrm{b}}^{-}\right) L^{\mathrm{b}-} D_{\mathrm{v}}^{++}\right), \\
& \widetilde{\mathcal{D}}_{\alpha}^{-}=L^{-+}\left(\mathcal{D}_{\alpha}^{-}-\left(\mathcal{D}_{\alpha}^{-} L_{\mathrm{b}}^{+}\right) L^{\mathrm{b}+} D_{\mathrm{v}}^{--}+\left(\mathcal{D}_{\alpha}^{-} L_{\mathrm{b}}^{+}\right) L^{\mathrm{b}-} D_{\mathrm{v}}^{0}\right)-L^{--} L^{-+} \widetilde{\mathcal{D}}_{\alpha}^{+} .
\end{aligned}
$$

The new derivatives $\widetilde{\mathcal{D}}_{\alpha}^{ \pm}$carry only $\mathrm{U}(1)_{\mathrm{w}}$ charge and are themselves twisted biholomorphic:

$$
\left[\mathcal{D}_{\mathrm{v}}^{++}, \widetilde{\mathcal{D}}_{\alpha}^{ \pm}\right]=\left[\mathcal{D}_{\mathrm{w}}^{--}, \widetilde{\mathcal{D}}_{\alpha}^{ \pm}\right]=\left[\mathcal{D}_{\mathrm{v}}^{0}, \widetilde{\mathcal{D}}_{\alpha}^{ \pm}\right]=0
$$

\footnotetext{
${ }^{12}$ If $L_{\mathrm{a}}{ }^{i}$ were a general superfield with $\operatorname{det} L_{\mathrm{a}}{ }^{i}=1$ but not obeying the constraint of a non-linear superfield, more complicated torsion terms would forbid analytic multiplets. This is one way of understanding why the $\mathrm{SU}(2)$ compensator must be a non-linear multiplet.

${ }^{13}$ It is also possible to further modify the definition (5.47) by an additional spin connection piece to eliminate the dimension-1/2 torsion. Doing so recovers precisely the supergeometry employed in [25, 26] (except for the central charge, which is easy to introduce). This supergeometry was introduced in [59, 60].
} 
They also obey the relations $\left[\widetilde{\mathcal{D}}^{++}, \widetilde{\mathcal{D}}_{\alpha}^{+}\right]=0$ and $\left[\widetilde{\mathcal{D}}^{--}, \widetilde{\mathcal{D}}_{\alpha}^{+}\right]=\widetilde{\mathcal{D}}_{\alpha}^{-}$and annihilate the harmonics and $L^{-+}$. It is not hard to see that in the central basis $\widetilde{\mathcal{D}}_{\alpha}^{ \pm}=U_{\mathrm{a}}^{ \pm} \widetilde{\mathcal{D}}_{\alpha}^{\mathrm{a}}$. These reduce in the gauge $L_{\mathrm{a}}{ }^{i}=\delta_{\mathrm{a}}{ }^{i}$ to the harmonic derivatives constructed directly in [25, 26].

To summarize: starting with the conformal superspace description, one can first translate all formula to $\mathrm{SU}(2)$ superspace, and then translate again to the Einstein superspace. Using the non-linear compensator, one can rewrite all formulae, including the component reduction formula, to be manifestly twisted biholomorphic. This completely eliminates any dependence on $z^{ \pm \pm}$and $z^{0}$, so that all fields and operators depend purely on the complex harmonics $u^{i \pm}$. Going to the gauge where $L_{\mathrm{a}}{ }^{i}=\delta_{\mathrm{a}}{ }^{i}$, the field dependent compensator $U^{\mathrm{a}+}$ can then be identified with the complex harmonics themselves and one recovers the covariant formulation of $[25,26]$. However, as has been found in a number of recent publications (see e.g. [11, 30] and references therein), the conformal superspace approach is often a more efficient scheme for analyzing superspace actions; therefore, we will refrain from giving the translations explicitly and remain with the manifestly superconformal framework.

There is one last issue which we would like to address within this section. Even within the superconformal framework, it is possible to interpret a non-linear multiplet as underlying the complex harmonic description [52]. Begin by choosing a gauge where $\mathcal{H}^{+4}$ vanishes. Because the independent auxiliary field $D$ of conformal supergravity is naturally found as the highest component of $\mathcal{H}^{+4}$, this gauge must amount to one where $D$ is composite exactly the indicator that one is employing a non-linear compensator. So where is the compensator if we haven't introduced it explicitly? As explained in [52], the vanishing of $\mathcal{H}^{+4}$ implies that $\nabla_{\mathrm{w}}^{++} \hat{z}^{++}=-\left(\hat{z}^{++}\right)^{2}$. Provided one has chosen $\nabla_{\alpha}^{+} \hat{z}^{++}=0$ without fixing any gauges but the coordinate choice, $\hat{z}^{++}$can be interpreted as a covariant non-linear primary superfield. What we wish to add to this old observation is that upon rewriting $\hat{z}^{++}$as

$$
\hat{z}^{++}=\frac{\hat{v}^{i+} w_{i}^{+}}{\hat{v}^{i+} w_{i}^{-}}=\frac{v^{j+} b_{j}{ }^{i} w_{i}^{+}}{v^{j+} b_{j}{ }^{i} w_{i}^{-}}
$$

in terms of the bridge superfield $b_{j}{ }^{i}$, one can show that the bridge $b_{j}{ }^{i}$ is actually harmonicindependent - it is precisely the non-linear multiplet compensator $L^{\mathrm{a} i}=-\delta_{k}^{\mathrm{a}} \epsilon^{i j} b_{j}{ }^{k}$.

\section{Superconformal sigma models from curved harmonic superspace}

As an application of the covariant harmonic superspace methods presented in earlier sections, we will derive the general component action for a hyperkähler sigma model coupled to conformal supergravity [44] using harmonic superspace methods. Because of the covariance of the approach, we can largely follow the same scheme used in rigid harmonic superspace. ${ }^{14}$ A similar calculation was performed by Ivanov and Valent using prepotential methods in [29]. ${ }^{15}$ The projective superspace version of this calculation appeared in [27].

Our starting point is the superconformal harmonic superspace Lagrangian (4.23). The conditions (4.24) are exactly those associated with a superconformal sigma model in har-

\footnotetext{
${ }^{14}$ See the pedagogical discussion in chapter 11 of the monograph [2].

${ }^{15}$ Ref. [29] addressed quaternionic sigma models coupled to supergravity with a hypermultiplet compensator. This is equivalent to hyperkähler sigma models coupled to conformal supergravity after gauge-fixing.
} 
monic superspace and will lead to a hyperkähler cone in the target space upon elimination of the auxiliary fields. Indices are raised and lowered with an $\operatorname{Sp}(n)$ matrix $\Omega_{\mathrm{ab}}$,

$$
Q^{\mathrm{a}+}=\Omega^{\mathrm{ab}} Q_{\mathrm{b}}^{+}, \quad Q_{\mathrm{a}}^{+}=Q^{\mathrm{b}+} \Omega_{\mathrm{ba}}, \quad \Omega^{\mathrm{ab}} \Omega_{\mathrm{bc}}=-\delta_{\mathrm{c}}^{\mathrm{a}} .
$$

In order to eliminate the auxiliary fields, we must solve their equations of motion. In the rigid approach, these were analyzed at the component level, but we will analyze them directly at the superfield level. Varying the Lagrangian (4.23) and discarding a total derivative, one finds

$$
\delta \mathscr{L}^{(2,2)}=-\delta Q^{\mathrm{a}+}\left(\nabla_{\mathrm{w}}^{++} Q_{\mathrm{a}}^{+}-\partial_{\mathrm{a}+} H^{(2,2)}\right)=0 .
$$

The term in parentheses must vanish, leading to the superfield equation of motion. It vanishes component-by-component in its $\theta^{+}$expansion as we place each component of $Q^{\text {a+ }}$ on-shell. Because we only wish to set the auxiliary fields on-shell, we should impose $\nabla_{\mathrm{w}}^{++} Q_{\mathrm{a}}^{+}=\partial_{\mathrm{a}+} H^{(2,2)}$ component-by-component except for the highest two components, which contain the equations of motion for the physical bosons and fermions.

Before proceeding with calculation, we first review how the hyperkähler geometry emerges, including the structure of gauged isometries, superconformal isometries, and supersymmetry transformations of the physical component fields. These properties are insensitive to whether the physical equations of motion are imposed or not, so we will be able to impose the full superfield equations of motion to aid our discussion. In addition, all of the formulae from here on will be taken in the central basis to simplify matters.

\subsection{Hyperkähler geometry and on-shell $\mathcal{N}=2$ superfields}

Harmonic superspace and hyperkähler geometry. The harmonic superspace approach to hyperkähler geometry was introduced in [61,62]. Here we provide only a concise summary of the results of these papers necessary for the evaluation of the component action of (4.23). The main difference with $[61,62]$ is that we will employ a complex harmonic formulation: that is, we complexify the real harmonics to $u_{i}^{+}=v_{i}^{+} / z^{0}$ and $u_{i}^{-}=w_{i}^{-}$, with various factors of $z^{0}=\left(v^{+}, w^{-}\right)$appearing as needed.

The lowest component of the superfield equation of motion (6.2) takes the form

$$
D_{\mathrm{w}}^{++} q_{\mathrm{a}}^{+}\left(\phi, v^{+}, w^{-}\right)=\partial_{\mathrm{a}+} H^{(2,2)}\left(q^{+}, v^{+}, w^{-}\right)
$$

and possesses a solution with $4 n$ real fields $\phi^{\mu}$ parametrizing a hyperkähler manifold $\mathcal{M}$. For now we will allow $H^{(2,2)}$ to depend also on $v^{i+}$; later on, we will discuss the specific features associated with cones. A special choice of coordinates is given by taking $\phi^{\mu}$ as the leading terms $f^{a i}$ in the harmonic expansion $q^{\text {a+ }}=z_{0}\left(f^{a i} u_{i}^{+}+\cdots\right)$; however, the specific choice of $\phi^{\mu}$ will not be relevant here. Once some choice is made, the geometry of the target space $\mathcal{M}$ can be summarized as follows. The solutions $q^{\mathrm{a}+}\left(\phi, v^{+}, w^{-}\right)$to $(6.3)$ can be used to construct a closed two-form

$$
\Omega^{++}=\frac{1}{2} \mathrm{~d} q^{\mathrm{a}+} \wedge \mathrm{d} q^{\mathrm{b}+} \Omega_{\mathrm{ab}}=\frac{1}{2} \mathrm{~d} q_{\mathrm{a}}^{+} \wedge \mathrm{d} q^{\mathrm{a}+}
$$


which is annihilated by $D_{\mathrm{w}}^{++}$. This means it possesses a terminating harmonic expansion $\Omega^{++}=\left(z_{0}\right)^{2} \Omega^{i j} u_{i}^{+} u_{j}^{+}=\Omega^{i j} v_{i}^{+} v_{j}^{+}$. The two-forms $\Omega_{\mu \nu}^{i j}$ are the three hyperkähler twoforms. Introducing $E_{\mu}{ }^{\mathrm{a}+}$ via $\mathrm{d} q^{\mathrm{a}+}=\mathrm{d} \phi^{\mu} E_{\mu}{ }^{\mathrm{a}+}$, we find

$$
\Omega^{++}=\frac{1}{2} \mathrm{~d} \phi^{\mu} \wedge \mathrm{d} \phi^{\nu} E_{\mu}^{\mathrm{a}+} E_{\nu}^{\mathrm{b}+} \Omega_{\mathrm{ab}} \quad \Longrightarrow \quad \Omega_{\mu \nu}{ }^{i j} u_{i}^{+} u_{j}^{+}=E_{\mu}{ }^{\mathrm{a}+} E_{\nu}^{\mathrm{b}+} \Omega_{\mathrm{ab}} .
$$

The harmonic-dependent function $E_{\mu}{ }^{a+}$ can be interpreted as (half of) a local vielbein on $\mathcal{M} \times T \mathbb{C} P^{1}$. Its local analytic $\operatorname{Sp}(n)$ structure group leaves the two-form $\Omega^{++}$invariant.

The form of (6.5) suggests the existence of a local $\operatorname{Sp}(n)$ transformation $L_{\mathrm{b}}{ }^{\mathrm{a}}\left(\phi, u^{ \pm}\right)$ whereby

$$
E_{\mu}{ }^{\mathrm{b}+} L_{\mathrm{b}}{ }^{\mathrm{a}}=e_{\mu}{ }^{\mathrm{a} i} v_{i}^{+}, \quad e_{\mu}^{\mathrm{a} i}=e_{\mu}{ }^{\mathrm{a} i}(\phi), \quad \Omega_{\mu \nu}{ }^{i j}=e_{\mu}{ }^{\mathrm{a}(i} e_{\nu}^{\mathrm{b} j)} \Omega_{\mathrm{ab}} .
$$

Noting that $D_{\mathrm{w}}^{++} e_{\mu}^{\mathrm{a}+}=0$, one can show that

$$
\mathcal{D}_{\mathrm{w}}^{++} E_{\mu}{ }^{\mathrm{a}+}:=D_{\mathrm{w}}^{++} E_{\mu}{ }^{\mathrm{a}+}-E_{\mu}{ }^{\mathrm{b}+} \omega^{(0,2)} \mathrm{b}^{\mathrm{a}}=0, \quad \omega^{(0,2)}{ }_{\mathrm{b}}^{\mathrm{a}}=L_{\mathrm{b}}{ }^{\mathrm{c}} D_{\mathrm{w}}^{++}\left(L^{-1}\right)_{\mathrm{c}}{ }^{\mathrm{a}},
$$

where $\mathcal{D}_{\mathrm{w}}^{++}$is interpreted as an $\operatorname{Sp}(n)$-covariant derivative in the analytic $\operatorname{Sp}(n)$ gauge with connection $\omega^{(0,2)} \mathrm{b}$. This connection can alternatively be specified in terms of $H^{(2,2)}$,

$$
\omega^{(0,2)} \mathrm{ab}=\partial_{\mathrm{a}+} \partial_{\mathrm{b}+} H^{(2,2)} .
$$

From the two different expressions for $\omega^{(0,2)}{ }_{\mathrm{ab}}$, the $\operatorname{Sp}(n)$ transformation $L_{\mathrm{b}}{ }^{\mathrm{a}}$ may be determined up to a harmonic-independent piece. This permits one to find the vielbein $e_{\mu}{ }^{a i}$, from which the hyperkähler metric $g_{\mu \nu}$ is constructed as

$$
g_{\mu \nu}=-e_{\mu}^{\mathrm{a} i} e_{\nu}^{\mathrm{b} j} \epsilon_{i j} \Omega_{\mathrm{ab}}=e_{\mu}^{\mathrm{a} i} e_{\nu \mathrm{a} i} .
$$

The usual vielbein postulate $\nabla_{\mu} e_{\nu}{ }^{\mathrm{a} i}=\partial_{\mu} e_{\nu}^{\mathrm{a} i}-\Gamma_{\mu \nu}{ }^{\rho} e_{\rho}^{\mathrm{a} i}-e_{\nu}{ }^{\mathrm{b} i} \omega_{\mu \mathrm{b}}{ }^{\mathrm{a}}=0$ (equivalently using the vanishing of the torsion tensor) allows one to determine the target space $\operatorname{Sp}(n)$ connection $\omega_{\mu \mathrm{b}}{ }^{\mathrm{a}}$ in the central $\operatorname{Sp}(n)$ gauge.

There is one additional piece of necessary information. The hyperkähler Riemann tensor $R_{\mu \nu \rho \sigma}$ possesses the tangent space decomposition

$$
R_{\mathrm{a} i \mathrm{bjc} k \mathrm{~d} l}:=e_{\mathrm{ai}}{ }^{\mu} e_{\mathrm{b} j}^{\nu} e_{\mathrm{ck}}{ }^{\rho} e_{\mathrm{d} l}^{\sigma} R_{\mu \nu \rho \sigma}=\epsilon_{i j} \epsilon_{k l} R_{\mathrm{abcd}}
$$

where $R_{\text {abcd }}$ is a totally symmetric $\operatorname{Sp}(n)$ tensor. Applying the local $\operatorname{Sp}(n)$ transformation $L_{\mathrm{a}}{ }^{\mathrm{b}}$, we find a new tensor

$$
\mathcal{R}_{\mathrm{abcd}}=L_{\mathrm{a}}{ }^{\mathrm{a}^{\prime}} L_{\mathrm{b}}{ }^{\mathrm{b}^{\prime}} L_{\mathrm{c}}{ }^{\mathrm{c}^{\prime}} L_{\mathrm{d}}{ }^{\mathrm{d}^{\prime}} R_{\mathrm{a}^{\prime} \mathrm{b}^{\prime} \mathrm{c}^{\prime} \mathrm{d}^{\prime}}, \quad \mathcal{D}_{\mathrm{w}}^{++} \mathcal{R}_{\mathrm{abcd}}=0
$$

One can show that $\mathcal{R}_{\text {abcd }}$ obeys

$$
\begin{aligned}
\left(z_{0}\right)^{-2} \mathcal{R}_{\mathrm{abcd}} & =H_{\mathrm{abcd}}^{(-2,2)}+3 \omega_{(\mathrm{ab}}^{-\mathrm{e}} H_{\mathrm{cd}) \mathrm{e}}^{(-1,2)}-\mathcal{D}_{\mathrm{w}}^{++} \mathcal{B}_{\mathrm{abcd}}^{--}, \\
H_{\mathrm{abc}}^{(-1,2)} & :=\partial_{\mathrm{a}+} \partial_{\mathrm{b}+} \partial_{\mathrm{c}+} H^{(2,2)}, \quad H_{\mathrm{abcd}}^{(-2,2)}:=\partial_{\mathrm{a}+} \partial_{\mathrm{b}+} \partial_{\mathrm{c}+} \partial_{\mathrm{d}+} H^{(2,2)},
\end{aligned}
$$


where $\omega_{\mathrm{abc}}^{-}$is the solution to the equation

$$
\mathcal{D}_{\mathrm{w}}^{++} \omega_{\mathrm{abc}}^{-}=H_{\mathrm{abc}}^{(-1,2)},
$$

and $\mathcal{B}_{\text {abcd }}^{--}$is a twisted biholomorphic quantity. ${ }^{16}$

Because these results follow from the equation of motion (6.3), they must hold as superfield equations provided (6.2) holds. Then we should find the superfields $Q^{\text {at }}$ are completely determined in terms of $4 n$ real superfields $\Phi^{\mu}$, so that any variation becomes

$$
\delta Q^{\mathrm{a}+}=\delta \Phi^{\mu} E_{\mu}{ }^{\mathrm{a}+} .
$$

In particular, this means that the two-form $\Omega^{++}$defined on the target space,

$$
\Omega^{++}=\frac{1}{2} \mathrm{~d} Q^{\mathrm{a}+} \wedge \mathrm{d} Q^{\mathrm{b}+} \Omega_{\mathrm{ab}}=\frac{1}{2} \mathrm{~d} \Phi^{\mu} \wedge \mathrm{d} \Phi^{\nu} e_{\mu}{ }^{\mathrm{a}+} e_{\nu}{ }^{\mathrm{b}+} \Omega_{\mathrm{ab}},
$$

may be generalized to any antisymmetrized variation

$$
\frac{1}{2} \delta Q^{\mathrm{a}+} \wedge \delta Q^{\mathrm{b}+} \Omega_{\mathrm{ab}}=\frac{1}{2} \delta \Phi^{\mu} \wedge \delta \Phi^{\nu} e_{\mu}{ }^{\mathrm{a}+} e_{\nu}{ }^{\mathrm{b}+} \Omega_{\mathrm{ab}} .
$$

Replacing $\delta$ with various local symmetry operations leads to useful results, some of which we will come across in the next few subsections.

Gauged isometries from harmonic superspace. We will be including the possibility of gauged isometries in the action. These were originally described in harmonic superspace in [63], whose results we summarize here. Suppose that the harmonic superspace Lagrangian possesses some isometries under which

$$
\delta Q^{\mathrm{a}+}=\lambda^{r} \mathcal{J}_{r}^{\mathrm{a}+}\left(Q^{+}, v^{+}, w^{-}\right),
$$

for constant parameters $\lambda^{r}$. In the superconformal case, $\mathcal{J}_{r}^{\mathrm{a}+}$ must actually be independent of $v^{i+}$, but we will remain with this more general case for the moment. In order for this to be an invariance of the action, it must obey

$$
\mathcal{J}_{r}^{\mathrm{a}+}=\Omega^{\mathrm{ab}} \partial_{\mathrm{b}+} D_{r}^{++}, \quad \mathcal{J}_{r}^{\mathrm{a}+} \partial_{\mathrm{a}+} H^{(2,2)}=\partial_{\mathrm{w}}^{++} D_{r}^{++} .
$$

for some biholomorphic function $D_{r}^{++}$of charge $(2,0)$, defined up to a shift by constant $c_{r}^{++}=c_{r}^{i j} v_{i}^{+} v_{j}^{+}$. The second equation in (6.18) determines the explicit dependence of $D_{r}^{++}$on $w_{i}^{-}$. When the equations of motion are imposed, so that $Q^{\text {a+ }}$ is determined as a function of the harmonics, the first condition of (6.18) is interpreted as the requirement of invariance of the hyperkähler two-form $\Omega^{++}$, while the second relation of (6.18) implies that $D_{r}^{++}$is $w_{i}^{-}$-independent,

$$
D_{\mathrm{w}}^{++} D_{r}^{++}=0 \quad \Longrightarrow \quad D_{r}^{++}=D_{r}^{i j} v_{i}^{+} v_{j}^{+} .
$$

The function $D_{r}^{++}$is the Killing potential (or moment map) of the hyperkähler manifold. On-shell, the gauge transformations of $Q^{\mathrm{a}+}$ must manifest on the fields $\phi^{\mu}$ of the target

\footnotetext{
${ }^{16}$ In the framework of $[61,62], \mathcal{B}_{\text {abcd }}^{--}=\mathcal{D}_{\mathrm{a}}^{-} \omega_{\mathrm{bcd}}^{-}$and $\omega_{\mathrm{abc}}^{-}$is the $\operatorname{Sp}(n)$ connection for the covariant target space derivative $\mathcal{D}_{\mathrm{a}}^{-}$in the analytic gauge.
} 
space as $\delta \phi^{\mu}=\lambda^{r} J_{r}{ }^{\mu}(\phi)$. As a consequence of (6.14), one finds $\mathcal{J}_{r}^{\mathrm{a}+}=J_{r}{ }^{\mu} E_{\mu}{ }^{\mathrm{a}+}$. From (6.18), one finds $\partial_{\mu} D_{r}{ }^{i j}=-\left(\Omega^{i j}\right)_{\mu \nu} J_{r}{ }^{\nu}$.

We are interested in situations where the Lagrangian (and not just the action) is gauge invariant and where it can be gauged in a manifestly covariant way - that is, by simply adding the appropriate connection to $D_{\mathrm{w}}^{++}$. In these cases, the Killing potential may be chosen in special form $D_{r}^{++}=-\frac{1}{2} Q_{\mathrm{a}}^{+} \mathcal{J}_{r}^{\mathrm{a}+}{ }^{17}$ When the Lagrangian is superconformal, this is always possible. By taking (6.16) and replacing one $\delta$ with a gauge transformation and the other with a dilatation, one may find the special form of the moment map on a hyperkähler cone, $D_{r}^{i j}=-\frac{1}{2} \chi^{\mu}\left(\Omega^{i j}\right)_{\mu \nu} J_{r}^{\nu}$.

Superconformal isometries and the hyperkähler potential. Now let us analyze the superconformal properties of the target space. The hypermultiplet $Q^{\mathrm{a}+}$ transforms locally under dilatations and $\mathrm{SU}(2)_{R}$ as

$$
\begin{aligned}
\delta Q^{\mathrm{a}+} & =\Lambda_{\mathrm{D}} \mathbb{D} Q^{\mathrm{a}+}+\lambda^{i}{ }_{j} I^{j}{ }_{i} Q^{\mathrm{a}+}=\left(\Lambda_{\mathrm{D}}+\lambda_{\mathrm{v}}^{-+}\right) Q^{\mathrm{a}+}-\lambda_{\mathrm{v}}^{++} \nabla_{\mathrm{v}}^{--} Q^{\mathrm{a}+}, \\
\lambda_{\mathrm{v}}^{++} & =\lambda^{i j} v_{i}^{+} v_{j}^{+}, \quad \lambda_{\mathrm{v}}^{+-}=\lambda^{i j} v_{i}^{+} v_{j}^{-},
\end{aligned}
$$

and is inert under $\mathrm{U}(1)_{R}$. On the target space, $\delta \Phi^{\mu}=\Lambda_{\mathrm{D}} k_{\mathrm{D}}^{\mu}+\lambda^{i}{ }_{j} k^{j}{ }_{i}^{\mu}$ for some choice of vectors $k_{\mathrm{D}}^{\mu}:=\mathbb{D} \Phi^{\mu}$ and $k_{i j}{ }^{\mu}:=I_{i j} \Phi^{\mu}$. Using (6.14), one may show that

$$
\begin{array}{rlrl}
k_{\mathrm{D}}^{\mu}{E_{\mu}}^{\mathrm{a}+} & =Q^{\mathrm{a}+}, \quad & \left(k_{\mathrm{v}}^{+-}\right)^{\mu} E_{\mu}{ }^{\mathrm{a}+} & =\frac{1}{2} Q^{\mathrm{a}+}, \\
\left(k_{\mathrm{v}}^{--}\right)^{\mu} E_{\mu}{ }^{\mathrm{a}+} & =D_{\mathrm{v}}^{--} Q^{\mathrm{a}+}, \quad\left(k_{\mathrm{v}}^{++}\right)^{\mu} E_{\mu}{ }^{\mathrm{a}+}=0 .
\end{array}
$$

These conditions imply that

$$
I^{i}{ }_{j} \Phi^{\mu} \equiv\left(k^{i}{ }_{j}\right)^{\mu}=\left(\Omega^{i}{ }_{j}\right)_{\nu}^{\mu} k_{\mathrm{D}}^{\nu} .
$$

Now using the transformation properties of the hyperkähler two-form, one can prove that $k_{\mathrm{D}}^{\mu}$ is a homothetic conformal Killing vector on the target space, $\nabla_{\mu} k_{\mathrm{D}}^{\nu}=\delta_{\mu}{ }^{\nu}$. In particular, there exists a globally-defined hyperkähler potential $K$ for which

$$
K=\frac{1}{2} k_{\mathrm{D}}^{\mu} k_{\mathrm{D} \mu}, \quad \partial_{\mu} K=k_{\mathrm{D} \mu} .
$$

There is a very useful alternative form for the hyperkähler potential:

$$
K=-Q_{\mathrm{a}}^{+} D_{\mathrm{v}}^{--} Q^{\mathrm{a}+} .
$$

To prove this agrees with (6.23), observe that when $Q^{\text {a+ }}$ obeys its equation of motion, $K$ obeys $D_{\mathrm{w}}^{++} K=0$ and so is harmonic independent. Now we use

$$
-Q_{\mathrm{a}}^{+} D_{\mathrm{v}}^{--} Q^{\mathrm{a}+}=-2\left(k_{\mathrm{v}}^{+-}\right)^{\mu}\left(k_{\mathrm{v}}^{--}\right)^{\nu} E_{\mu \mathrm{a}}^{+} E_{\nu}{ }^{\mathrm{a}+}=-2\left(k_{\mathrm{v}}^{+-}\right)^{\mu}\left(k_{\mathrm{v}}^{--}\right)^{\nu} \Omega_{\mu \nu}{ }^{++}=\frac{1}{2} k_{\mathrm{D}}^{\mu} k_{\mathrm{D} \mu}
$$

after applying (6.22), and so the equality between (6.23) and (6.24) follows. This expression also follows by replacing the variations in $(6.16)$ with $D_{\mathrm{v}}^{0}$ and $D_{\mathrm{v}}^{--}$: in other words, the function $K$ is a component of the pullback of $\Omega^{++}$to the complex harmonic manifold.

\footnotetext{
${ }^{17}$ This choice for the Killing potential is actually always possible provided we do not adopt the special gauge $L_{\mathrm{a}}^{+}=Q_{\mathrm{a}}^{+}$. As is familiar from Kähler target spaces in $\mathcal{N}=1$ theories [64], it is possible to introduce non-dynamical multiplets with vanishing kinetic terms whose sole purpose is to render the Lagrangian completely gauge invariant, so that minimal substitution may proceed. The cost of this approach in harmonic superspace would be zero eigenvalues of the harmonic "kinetic matrix" $\partial_{[\mathrm{a}+} L_{\mathrm{b}]}^{+}$.
} 
Supersymmetry and fermion transformations. Up until now, we have discussed only the physical bosonic field $\phi^{\mu}$ and its superfield lift $\Phi^{\mu}$. The sigma model also involves an $\operatorname{Sp}(n)$ fermion $\zeta_{\underline{\alpha}}{ }^{\mathrm{b}}$ related to $\phi$ by supersymmetry. Here we will review how it emerges from the harmonic structure. Following [2], we define the fermion $\Psi_{\underline{\alpha}}{ }^{\mathrm{a}}:=\nabla_{\underline{\alpha}}^{-} Q^{\mathrm{a}+} \mid$, which is twisted biholomorphic and obeys

$$
\mathcal{D}_{\mathrm{w}}^{++} \Psi_{\underline{\alpha}}^{\mathrm{a}}:=D_{\mathrm{w}}^{++} \Psi_{\underline{\alpha}}^{\mathrm{a}}-\Psi_{\underline{\alpha}}^{\mathrm{b}} \omega^{(0,2)}{ }_{\mathrm{b}}^{\mathrm{a}}=0
$$

when $Q^{\text {a+ }}$ is on-shell. This implies that we can introduce the harmonic-independent physical fermions $\zeta_{\underline{\alpha}}{ }^{\mathrm{a}}$ via $\Psi_{\underline{\alpha}^{\mathrm{a}}}^{\mathrm{a}} \equiv \zeta_{\underline{\alpha}}^{\mathrm{b}}\left(L^{-1}\right)_{\mathrm{b}}{ }^{\mathrm{a}}$ In accordance with (6.14), one finds that

$$
\nabla_{\alpha}^{-} \Phi^{\mu}\left|E_{\mu}{ }^{\mathrm{a}+}=\zeta_{\underline{\alpha}}^{\mathrm{b}}\left(L^{-1}\right)_{\mathrm{b}}^{\mathrm{a}} \quad \Longrightarrow \quad \nabla_{\underline{\alpha} i} \Phi^{\mu}\right|=\zeta_{\underline{\alpha}}{ }^{\mathrm{a}} e_{\mathrm{a} i}{ }^{\mu},
$$

which implies the supersymmetry transformations

$$
\delta_{\mathrm{Q}} \phi^{\mu}=\xi_{i} \zeta^{\mathrm{b}} e_{\mathrm{b}}^{i \mu}+\bar{\xi}^{i} \bar{\zeta}^{\mathrm{b}} e_{\mathrm{b} i}^{\mu}
$$

where $\xi_{i}^{\alpha}$ and $\bar{\xi}_{\dot{\alpha}}^{i}$ are the SUSY parameters. The target space vielbeine $e_{\mu}{ }^{a i}$ and their inverses $e_{\mathrm{a} i}{ }^{\mu}$ coincide with corresponding quantities introduced in [65, 66] (see also [67] and [44]). The fermions obey the condition $\left(\zeta_{\alpha}{ }^{\mathrm{b}}\right)^{*}=\bar{\zeta}_{\dot{\alpha} \mathrm{b}}$.

In a similar way, we can calculate the supersymmetry and $S$-supersymmetry transformations of the fermions,

$$
\begin{aligned}
& \delta \zeta_{\alpha}^{\mathrm{a}}=-2 i \nabla_{\alpha \dot{\beta}} \phi^{\mu} e_{\mu i}{ }^{\mathrm{a}} \bar{\xi}^{\dot{\beta} i}-2 \bar{W}^{r} J_{r}{ }^{i \mathrm{a}} \xi_{\alpha i}+4 \eta_{\alpha}^{i} A_{i}{ }^{\mathrm{a}}+\delta \phi^{\mu} \zeta_{\alpha}^{\mathrm{c}} \omega_{\mu \mathrm{c}}{ }^{\mathrm{a}} \\
& \delta \bar{\zeta}_{\mathrm{a}}^{\dot{\alpha}}=+2 i \nabla^{\dot{\alpha} \beta} \phi^{\mu} e_{\mu \mathrm{a}}{ }^{i} \xi_{\beta i}-2 W^{r} J_{r i \mathrm{a}} \bar{\xi}^{\dot{\alpha} i}-4 \bar{\eta}_{i}^{\dot{\alpha}} A_{\mathrm{a}}^{i}-\delta \phi^{\mu} \bar{\zeta}_{\mathrm{c}}^{\dot{\alpha}} \omega_{\mu \mathrm{a}}{ }^{\mathrm{c}}
\end{aligned}
$$

where $\eta^{i \alpha}$ and $\bar{\eta}_{i \dot{\alpha}}$ are the $S$-supersymmetry parameters and $W^{r}$ is the complex scalar of the vector multiplet. We employ the same conventions for the vector multiplet as [10]. We have introduced the pseudoreal $\operatorname{Sp}(\mathrm{n}) \times \mathrm{Sp}(1)$ sections $A^{i \mathrm{a}}$ associated with the conformal Killing vectors [44]

$$
A^{i \mathrm{a}}:=k_{\mathrm{D}}^{\mu} e_{\mu}^{i \mathrm{a}}, \quad\left(A^{i \mathrm{a}}\right)^{*}=A_{i \mathrm{a}}, \quad A^{\mathrm{a}+}=Q^{\mathrm{b}+} L_{\mathrm{b}}{ }^{\mathrm{a}} .
$$

It is helpful to note that $\nabla_{a} \phi^{\mu} e_{\mu}{ }^{a i}=\hat{\nabla}_{a} A^{\text {ai }}$ where $\hat{\nabla}_{a}$ also carries the $\operatorname{Sp}(n)$ connection. For reference, we also give the transformations of the fermions under gauged isometries,

$$
\begin{aligned}
\delta_{g} \zeta_{\alpha}^{\mathrm{a}} & =\frac{1}{2} \lambda^{r} \zeta_{\alpha}^{\mathrm{b}} e_{\mathrm{b} j}{ }^{\mu}\left(\nabla_{\mu} J_{r}^{\nu}\right) e_{\nu}{ }^{\mathrm{a} \mathrm{a}}+\delta_{g} \phi^{\mu} \zeta_{\alpha}^{\mathrm{c}} \omega_{\mu \mathrm{c}}^{\mathrm{a}}, \\
\delta_{g} \bar{\zeta}_{\mathrm{a}}^{\dot{\alpha}} & =\frac{1}{2} \lambda^{r} \bar{\zeta}_{\mathrm{b}}^{\dot{\alpha}} e^{\mathrm{b} j \mu}\left(\nabla_{\mu} J_{r}^{\nu}\right) e_{\nu j \mathrm{a}}-\delta_{g} \phi^{\mu} \bar{\zeta}_{\mathrm{c}}^{\dot{\alpha}} \omega_{\mu \mathrm{a}}{ }^{\mathrm{c}}
\end{aligned}
$$

Some useful identities. Finally, we will need some useful identities arising from (6.16). For example, taking the spinor part of the pullback of $\Omega^{++}$to $\mathcal{M}^{4 \mid 8}$ leads to

$$
\Omega_{\underline{\alpha} \underline{\beta}}=\nabla_{\underline{\alpha}}^{-} Q_{\mathrm{a}}^{+}\left|\nabla_{\underline{\beta}}^{-} Q^{\mathrm{a}+}\right|=\zeta_{\underline{\alpha} \mathrm{a}} \zeta_{\underline{\beta}}^{\mathrm{a}} .
$$

Each of the quantities $\Omega_{\alpha \beta}, \Omega_{\alpha \dot{\beta}}$ and $\Omega_{\dot{\alpha} \dot{\beta}}$ are harmonic-independent at lowest order in their $\theta$ expansion. Similar expressions arise using the vector derivatives or the gauge generator,

$$
\Omega_{\underline{\alpha} b}^{+}:=\nabla_{\underline{\alpha}}^{-} Q_{\mathrm{a}}^{+}\left|\nabla_{b} Q^{\mathrm{a}+}\right|=\zeta_{\underline{\alpha} a} \widehat{\nabla}_{b} A^{\mathrm{a}+}, \quad \Omega_{\underline{\alpha} r}^{+}:=\nabla_{\underline{\alpha}}^{-} Q_{\mathrm{a}}^{+}\left|X_{r} Q^{\mathrm{a}+}\right|=\zeta_{\underline{\alpha} a} J_{r}^{\mathrm{a}+} .
$$


Other expressions such as $\Omega_{r b}^{++}$or $\Omega_{a b}^{++}$could be introduced, but we won't need them. We will however need spinor derivatives of the hyperkähler potential and moment map:

$$
\begin{aligned}
\nabla_{\underline{\alpha}}^{+} K \mid & =Q_{\mathrm{a}}^{+} \nabla_{\underline{\alpha}}^{-} Q^{\mathrm{a}+}\left|=A_{\mathrm{a}}^{+} \zeta_{\underline{\alpha}}^{\mathrm{a}}, \quad \nabla_{\underline{\alpha}}^{-} K\right|=A_{\mathrm{a}}^{-} \zeta_{\underline{\alpha}}^{\mathrm{a}}=D_{\mathrm{v}}^{--} Q_{\mathrm{a}}^{+} \nabla_{\underline{\alpha}}^{-} Q^{\mathrm{a}+} \mid, \\
\nabla_{\underline{\alpha}}^{-} D_{r}^{++} \mid & =-\Omega_{\underline{\alpha} r}^{+} .
\end{aligned}
$$

\subsection{The component fields of $Q^{\mathrm{a}+}$ and auxiliary equations of motion}

Now we are prepared to set up the component calculation. Begin by defining the components of $Q^{\mathrm{a}+}$, largely following [2]:

$$
\begin{aligned}
\Psi_{\alpha}^{\mathrm{a}} & :=\nabla_{\alpha}^{-} Q^{\mathrm{a}+} \mid, & \Psi_{\dot{\alpha}}^{\mathrm{a}} & :=\bar{\nabla}_{\dot{\alpha}}^{-} Q^{\mathrm{a}+} \mid, \\
M^{\mathrm{a}-} & :=-\frac{1}{4}\left(\nabla^{-}\right)^{2} Q^{\mathrm{a}+} \mid, & N^{\mathrm{a}-} & :=-\frac{1}{4}\left(\bar{\nabla}^{-}\right)^{2} Q^{\mathrm{a}+} \mid, \\
A_{\alpha \dot{\beta}}^{\mathrm{a}-} & :=-i \nabla_{\alpha}^{-} \nabla_{\dot{\beta}}^{-} Q^{\mathrm{a}+} \mid, & & \\
\Xi_{\alpha}^{\mathrm{a}--} & :=\frac{1}{8} \nabla_{\alpha}^{-}\left(\bar{\nabla}^{-}\right)^{2} Q^{\mathrm{a}+} \mid, & \Xi_{\dot{\alpha}}^{\mathrm{a}--} & :=\frac{1}{8} \bar{\nabla}_{\dot{\alpha}}^{-}\left(\nabla^{-}\right)^{2} Q^{\mathrm{a}+} \mid, \\
P^{\mathrm{a}(-3)} & :=\frac{1}{16}\left(\nabla^{-}\right)^{2}\left(\bar{\nabla}^{-}\right)^{2} Q^{\mathrm{a}+} \mid . & &
\end{aligned}
$$

These will be the most convenient definitions for the component calculation we will soon undertake, but they possess one important disadvantage that must be kept in mind. Each of the terms aside from $\Psi_{\underline{\alpha}}^{\mathrm{a}}$ is not twisted biholomorphic. In particular, one can show that

$$
D_{\mathrm{v}}^{++} A_{b}{ }^{\mathrm{a}-}=-2 \nabla_{b} q^{\mathrm{a}+}, \quad D_{\mathrm{v}}^{++} M^{\mathrm{a}-}=-\bar{W}^{r} \mathcal{J}_{r}{ }^{\mathrm{a}+}, \quad D_{\mathrm{v}}^{++} N^{\mathrm{a}-}=-W^{r} \mathcal{J}_{r}{ }^{\mathrm{a}+} .
$$

The expressions for $D_{\mathrm{v}}^{++} \Xi_{\alpha}^{\mathrm{a}--}$ and $D_{\mathrm{v}}^{++} P^{\mathrm{a}(-3)}$ are more complicated and can be derived from similar formulae given in $[10]$

$$
\begin{aligned}
D_{\mathrm{v}}^{++} \Xi_{\alpha}^{\mathrm{a}--}= & -\frac{i}{2} \nabla_{\alpha \dot{\beta}} \bar{\nabla}^{\dot{\beta}-} Q^{\mathrm{a}+}+2 \lambda_{\alpha}^{r-} \mathcal{J}_{r}^{\mathrm{a}+}+\frac{1}{2} W^{r} \nabla_{\alpha}^{-} \mathcal{J}_{r}^{\mathrm{a}+} \\
& -\frac{1}{2} W_{\alpha}^{\beta} \nabla_{\beta}^{-} Q^{\mathrm{a}+}-\frac{3}{2} \chi_{\alpha}^{-} Q^{\mathrm{a}+}+\frac{3}{2} \chi_{\alpha}^{+} D^{--} Q^{\mathrm{a}+}, \\
D_{\mathrm{v}}^{++} P^{\mathrm{a}(-3)}= & -\frac{i}{2} \nabla^{\dot{\alpha} \alpha} \nabla_{\alpha}^{-} \nabla_{\dot{\alpha}}^{-} Q^{\mathrm{a}+}-3 D D^{--} Q^{\mathrm{a}+} \\
& +\frac{3}{2} \chi^{\alpha+} D^{--} \nabla_{\alpha}^{-} Q^{\mathrm{a}+}-\frac{3}{2} \bar{\chi}_{\dot{\alpha}}^{+} D^{--} \bar{\nabla}^{\dot{\alpha}-} Q^{\mathrm{a}+} \\
& +2 \lambda^{\alpha r-} \nabla_{\alpha}^{-} \mathcal{J}_{r}^{\mathrm{a}+}-2 \bar{\lambda}_{\dot{\alpha}}^{r-} \bar{\nabla}^{\dot{\alpha}-} \mathcal{J}_{r}^{\mathrm{a}+} \\
& +\frac{1}{4} \bar{W}^{r}\left(\bar{\nabla}^{-}\right)^{2} \mathcal{J}_{r}^{\mathrm{a}+}+\frac{1}{4} W^{r}\left(\nabla^{-}\right)^{2} \mathcal{J}_{r}^{\mathrm{a}+}+3 Y^{r--} \mathcal{J}_{r}^{\mathrm{a}+} .
\end{aligned}
$$

The vector multiplet gaugino is denoted $\lambda_{\alpha i}{ }^{r}$ and the pseudo-real auxiliary is $Y_{i j}{ }^{r}$, again following [10]. To keep notation simple, we have omitted the explicit component projection.

The equations of motion of the component fields correspond to the action of $D_{\mathrm{w}}^{++}$ and can be derived by successively taking spinor derivatives of the superfield equation of motion (6.2). They are summarized in table 1 . We impose these only through the $\theta^{2}$ level:

$$
D_{\mathrm{w}}^{++} q_{\mathrm{a}}^{+}=\partial_{\mathrm{a}+} H^{(2,2)},
$$




\begin{tabular}{|clc|}
\hline component field & $\Longrightarrow$ & equation of motion \\
\hline$P^{\mathrm{a}(-3)}$ & $\Longrightarrow$ & $D_{\mathrm{w}}^{++} q_{\mathrm{a}}^{+}=\partial_{\mathrm{a}+} H^{(2,2)}$ \\
$\Xi_{\underline{\alpha}}^{\mathrm{a}--}$ & $\Longrightarrow$ & $D_{\mathrm{w}}^{++} \Psi_{\underline{\alpha} \mathrm{a}}=\nabla_{\underline{\alpha}}^{-}\left(\partial_{\mathrm{a}+} H^{(2,2)}\right) \mid$ \\
$N^{\mathrm{a}-}$ & $\Longrightarrow$ & $D_{\mathrm{w}}^{++} M_{\mathrm{a}}^{-}=-\frac{1}{4}\left(\nabla^{-}\right)^{2}\left(\partial_{\mathrm{a}+} H^{(2,2)}\right) \mid$ \\
$A_{\alpha \dot{\alpha}}^{\mathrm{a}-}$ & $\Longrightarrow$ & $D_{\mathrm{w}}^{++} A_{\alpha \dot{\alpha} \mathrm{b}}^{-}=-i \nabla_{\alpha}^{-} \bar{\nabla}_{\dot{\alpha}}^{-}\left(\partial_{\mathrm{b}+} H^{(2,2)}\right) \mid$ \\
$M^{\mathrm{a}-}$ & $\Longrightarrow$ & $D_{\mathrm{w}}^{++} N_{\mathrm{a}}^{-}=-\frac{1}{4}\left(\bar{\nabla}^{-}\right)^{2}\left(\partial_{\mathrm{a}+} H^{(2,2)}\right) \mid$ \\
$\Psi_{\alpha}^{\mathrm{a}}$ & $\Longrightarrow$ & $D_{\mathrm{w}}^{++} \Xi_{\alpha \mathrm{b}}^{--}=\frac{1}{8} \nabla_{\alpha}^{-}\left(\bar{\nabla}^{-}\right)^{2}\left(\partial_{\mathrm{a}+} H^{(2,2)}\right) \mid$ \\
$q^{\mathrm{a}+}$ & $\Longrightarrow$ & $D_{\mathrm{w}}^{++} P_{\mathrm{a}}^{(-3)}=\left(\nabla^{-}\right)^{4}\left(\partial_{\mathrm{a}+} H^{(2,2)}\right) \mid$ \\
\hline
\end{tabular}

Table 1. Component field equations of motion.

$$
\begin{aligned}
& \mathcal{D}_{\mathrm{w}}^{++} \Psi_{\underline{\alpha} \mathrm{a}} \equiv D_{\mathrm{w}}^{++} \Psi_{\underline{\alpha} \mathrm{a}}-\Psi_{\underline{\alpha}}^{\mathrm{b}} H_{\mathrm{ba}}^{(0,2)}=0, \\
& \mathcal{D}_{\mathrm{w}}^{++} M_{\mathrm{a}}^{-} \equiv D_{\mathrm{w}}^{++} M_{\mathrm{a}}^{-}-M^{\mathrm{b}-} H_{\mathrm{ba}}^{(0,2)}=-\frac{1}{4} \Psi^{\mathrm{b}} \Psi^{\mathrm{c}} H_{\mathrm{abc}}^{(-1,2)}, \\
& \mathcal{D}_{\mathrm{w}}^{++} N_{\mathrm{a}}^{-} \equiv D_{\mathrm{w}}^{++} N_{\mathrm{a}}^{-}-N^{\mathrm{b}-} H_{\mathrm{ba}}^{(0,2)}=-\frac{1}{4} \bar{\Psi}^{\mathrm{b}} \bar{\Psi}^{\mathrm{c}} H_{\mathrm{abc}}^{(-1,2)}, \\
& \mathcal{D}_{\mathrm{w}}^{++} A_{\alpha \dot{\alpha} \mathrm{a}}^{-} \equiv D_{\mathrm{w}}^{++} A_{\alpha \dot{\alpha} \mathrm{a}}^{-}-A_{\alpha \dot{\alpha}}^{\mathrm{b}-} H_{\mathrm{ba}}^{(0,2)}=-i \Psi_{\alpha}^{\mathrm{b}} \bar{\Psi}_{\dot{\alpha}}^{\mathrm{c}} H_{\mathrm{abc}}^{(-1,2)} .
\end{aligned}
$$

The solutions completely determine the harmonic expansions of $q^{\mathrm{a}+}, \Psi_{\underline{\alpha}}^{\mathrm{a}}, M^{\mathrm{a}-}, N^{\mathrm{a}-}$, and $A_{b}{ }^{\mathrm{a}-}$ in terms of the physical fields $\phi^{\mu}$ and $\zeta_{\underline{\alpha}}^{\mathrm{b}}$. We have already explained how these arise in $q^{\text {at }}$ and $\Psi_{\underline{\alpha}}^{\mathrm{a}}$. For the others, we find

$$
\begin{aligned}
& M_{\mathrm{a}}^{-}=-\bar{W}^{r} \mathcal{J}_{r \mathrm{a}}^{-}-\frac{z^{--}}{\left(z^{0}\right)^{2}} \bar{W}^{r} \mathcal{J}_{r \mathrm{a}}^{+}-\frac{1}{4} \Psi^{\mathrm{b}} \Psi^{\mathrm{c}} \omega_{\mathrm{abc}}^{-}, \\
& N_{\mathrm{a}}^{-}=-W^{r} \mathcal{J}_{r \mathrm{a}}^{-}-\frac{z^{--}}{\left(z^{0}\right)^{2}} W^{r} \mathcal{J}_{r \mathrm{a}}^{+}-\frac{1}{4} \bar{\Psi}^{\mathrm{b}} \bar{\Psi}^{\mathrm{c}} \omega_{\mathrm{abc}}^{-}, \\
& A_{\alpha \dot{\alpha}}^{\mathrm{a}-}=-2 \nabla_{\alpha \dot{\alpha}} \phi^{\mu} E_{\mu}{ }^{\mathrm{a}-}-2 \frac{z^{--}}{\left(z^{0}\right)^{2}} \nabla_{\alpha \dot{\alpha}} q^{\mathrm{a}+}-i \Psi_{\alpha}^{\mathrm{b}} \bar{\Psi}_{\dot{\alpha}}^{\mathrm{c}} \omega_{\mathrm{bc}}^{-\mathrm{a}},
\end{aligned}
$$

where $\mathcal{J}_{r}^{\mathrm{a}-}$ and ${E_{\mu}}^{\mathrm{a}-}$ are twisted biholomorphic solutions to the equations

$$
\left(z^{0}\right)^{2} \mathcal{D}_{\mathrm{w}}^{++} \mathcal{J}_{r}^{\mathrm{a}-}=\mathcal{J}_{r}^{\mathrm{a}+}, \quad\left(z^{0}\right)^{2} \mathcal{D}_{\mathrm{w}}^{++} E_{\mu}{ }^{\mathrm{a}-}=E_{\mu}{ }^{\mathrm{a}+} .
$$

Note that they are chosen to carry $\mathrm{U}(1)_{\mathrm{v}}$ charge. This means that

$$
E_{\mu}{ }^{\mathrm{b}+} L_{\mathrm{b}}{ }^{\mathrm{a}}=e_{\mu}{ }^{\mathrm{a} i} v_{i}^{+}, \quad E_{\mu}{ }^{\mathrm{b}-} L_{\mathrm{b}}{ }^{\mathrm{a}}=e_{\mu}{ }^{\mathrm{a} i} \frac{w_{i}^{-}}{z^{0}}, \quad \mathcal{J}_{r}{ }^{\mathrm{a} \pm}=J_{r}{ }^{\mu} E_{\mu}{ }^{\mathrm{a} \pm} .
$$

\subsection{Summary of the component calculation}

All the pieces are now in place to work out the component reduction. Here one important observation will drastically simplify the analysis: when $Q^{\text {a+ }}$ is placed fully on-shell, the superconformal Lagrangian $\mathscr{L}^{(2,2)}$ completely vanishes as a consequence of the homogeneity of $H^{(2,2)}$. Now we are only imposing the equations of motion to the $\theta^{2}$ level, so this means 
that in evaluating the component Lagrangian we only need to keep the terms at higher order than $\theta^{2}$. (This same observation simplified the projective superspace calculation of [27].) Thus the component reduction formula (4.18) simplifies to evaluating two terms:

$$
\begin{aligned}
S & =\frac{i}{2 \pi} \int \mathrm{d}^{4} x e \int_{\mathcal{S}} \mathcal{V}^{++} \wedge \mathcal{W}^{--}\left(T_{0}^{(-2,2)}+T_{1}^{(-2,2)}\right), \\
T_{0}^{(-2,2)} & =\frac{1}{16}\left(\nabla^{-}\right)^{2}\left(\bar{\nabla}^{-}\right)^{2} \mathscr{L}^{(2,2)}\left|, \quad T_{1}^{(-2,2)}=-\frac{i}{8}\left(\bar{\psi}_{m}^{-} \bar{\sigma}^{m}\right)^{\alpha} \nabla_{\alpha}^{-}\left(\bar{\nabla}^{-}\right)^{2} \mathscr{L}^{(2,2)}\right|+\text { h.c. }
\end{aligned}
$$

Let us begin with the leading order term. Imposing the superfield equation of motion through the $\theta^{2}$ level, one can show (recalling that $D_{\mathrm{w}}^{++}$commutes with $\nabla_{\underline{\alpha}}^{-}$)

$$
\begin{aligned}
T_{0}^{(-2,2)}= & \frac{1}{2} D_{\mathrm{w}}^{++}\left(q_{\mathrm{a}}^{+} P^{\mathrm{a}(-3)}\right)+\frac{1}{2} D_{\mathrm{w}}^{++}\left(\Psi_{\mathrm{a}}^{\alpha} \Xi_{\alpha}^{\mathrm{a}--}+\text { h.c. }\right) \\
& -\frac{1}{8} H_{\mathrm{abc}}^{(-1,2)}\left(\Psi^{\mathrm{a}} \Psi^{\mathrm{b}} N^{\mathrm{c}-}+\bar{\Psi}^{\mathrm{a}} \bar{\Psi}^{\mathrm{b}} M^{\mathrm{c}-}\right)+\frac{i}{8} H_{\mathrm{abc}}^{(-1,2)} \Psi_{\alpha}^{\mathrm{a}} \bar{\Psi}_{\dot{\alpha}}^{\mathrm{b}} A^{\dot{\alpha} \alpha \mathrm{c}-} \\
& +\frac{1}{16} H_{\mathrm{abcd}}^{(-2,2)} \Psi^{\mathrm{a}} \Psi^{\mathrm{b}} \bar{\Psi}^{\mathrm{c}} \bar{\Psi}^{\mathrm{d}} .
\end{aligned}
$$

Using (6.12) and (6.13), this can be rewritten

$$
\begin{aligned}
T_{0}^{(-2,2)}= & \frac{1}{2} D_{\mathrm{w}}^{++}\left(q_{\mathrm{a}}^{+} P^{\mathrm{a}(-3)}\right)+\frac{1}{2} D_{\mathrm{w}}^{++}\left(\Psi_{\mathrm{a}}^{\alpha} \Xi_{\alpha}^{\mathrm{a}--}+\text { h.c. }\right) \\
& +\frac{1}{16}\left(z^{0}\right)^{-2} \mathcal{R}_{\mathrm{abcd}} \Psi^{\mathrm{a}} \Psi^{\mathrm{b}} \bar{\Psi}^{\mathrm{c}} \bar{\Psi}^{\mathrm{d}}+\frac{1}{16} D_{\mathrm{w}}^{++}\left(\mathcal{B}_{\mathrm{abcd}} \Psi^{\mathrm{a}} \Psi^{\mathrm{b}} \bar{\Psi}^{\mathrm{c}} \bar{\Psi}^{\mathrm{d}}\right) \\
& +\frac{1}{8} D_{\mathrm{w}}^{++}\left[\omega_{\mathrm{abc}}^{-}\left(i \Psi_{\alpha}^{\mathrm{a}} \bar{\Psi}_{\dot{\alpha}}^{\mathrm{b}} A^{\dot{\alpha} \alpha \mathrm{c}-}-\Psi^{\mathrm{a}} \Psi^{\mathrm{b}} N^{\mathrm{c}-}-\bar{\Psi}^{\mathrm{a}} \bar{\Psi}^{\mathrm{b}} M^{\mathrm{c}-}\right)\right] \\
& -\frac{1}{8} \omega_{\mathrm{abc}}^{-}\left(i \Psi_{\alpha}^{\mathrm{a}} \bar{\Psi}_{\dot{\alpha}}^{\mathrm{b}} \mathcal{D}_{\mathrm{w}}^{++} A^{\dot{\alpha} \alpha \mathrm{c}-}-\Psi^{\mathrm{a}} \Psi^{\mathrm{b}} \mathcal{D}_{\mathrm{w}}^{++} N^{\mathrm{c}-}-\bar{\Psi}^{\mathrm{a}} \bar{\Psi}^{\mathrm{b}} \mathcal{D}_{\mathrm{w}}^{++} M^{\mathrm{c}-}\right) \\
& -\frac{1}{32} \omega_{\mathrm{ab}}^{-\mathrm{e}} H_{\mathrm{cde}}^{(-1,2)} \Psi^{\mathrm{a}} \Psi^{\mathrm{b}} \bar{\Psi}^{\mathrm{c}} \bar{\Psi}^{\mathrm{d}}-\frac{1}{32} \omega_{\mathrm{ab}}^{-\mathrm{e}} H_{\mathrm{cde}}^{(-1,2)} \bar{\Psi}^{\mathrm{a}} \bar{\Psi}^{\mathrm{b}} \Psi^{\mathrm{c}} \Psi^{\mathrm{d}} \\
& -\frac{1}{8} \omega_{\mathrm{ac}}^{-\mathrm{e}} H_{\mathrm{bde}}^{(-1,2)} \Psi^{\mathrm{a}} \Psi^{\mathrm{b}} \bar{\Psi}^{\mathrm{c}} \bar{\Psi}^{\mathrm{d}} .
\end{aligned}
$$

Imposing the equations of motion for the auxiliaries, we find that the last three lines cancel. Furthermore, $\mathcal{R}_{\text {abcd }} \Psi^{\mathrm{a}} \Psi^{\mathrm{b}} \bar{\Psi}^{\mathrm{c}} \bar{\Psi}^{\mathrm{d}}$ simplifies to the harmonic-independent $R_{\text {abcd }} \zeta^{\mathrm{a}} \zeta^{\mathrm{b}} \bar{\zeta}^{\mathrm{c}} \bar{\zeta}^{\mathrm{d}}$. All of these manipulations correspond so far (as they must) to the rigid harmonic superspace calculation [2]. Now we need to integrate by parts. Using (2.14b), we can write

$$
\frac{i}{2 \pi} \int \mathcal{V}^{++} \wedge \mathcal{W}^{--} T_{0}^{(-2,2)}=\frac{i}{2 \pi} \int \mathcal{V}^{++} \wedge \mathcal{V}^{--} T_{0}^{\prime}
$$

for some new quantity $T_{0}^{\prime}$ given by

$$
\begin{aligned}
T_{0}^{\prime}= & -\frac{1}{4} q_{\mathrm{a}}^{+} \nabla^{\dot{\alpha} \alpha} A_{\alpha \dot{\alpha}}^{\mathrm{a}-}+\left(\frac{i}{4} \Psi_{\mathrm{a}}^{\alpha} \widehat{\nabla}_{\alpha \dot{\alpha}} \bar{\Psi}^{\dot{\alpha} \mathrm{a}}+\text { h.c. }\right)+\frac{1}{16} R_{\mathrm{abcd}} \zeta^{\mathrm{a}} \zeta^{\mathrm{b}} \bar{\zeta}^{\mathrm{c}} \bar{\zeta}^{\mathrm{d}} \\
& -\frac{i}{4} \omega_{\mathrm{abc}}^{-} \Psi_{\alpha}^{\mathrm{a}} \bar{\Psi}_{\dot{\alpha}}^{\mathrm{b}} \nabla^{\dot{\alpha} \alpha} q^{\mathrm{c}+}-\frac{1}{8} \omega_{\mathrm{abc}}^{-}\left(\Psi^{\mathrm{a}} \Psi^{\mathrm{b}} W^{r} J_{r}^{\mathrm{c}+}+\text { h.c. }\right) \\
& +\frac{3}{2} D q_{\mathrm{a}}^{+} D_{\mathrm{v}}^{--} q^{\mathrm{a}+}-\frac{1}{4} W^{\alpha \beta} \Psi_{\mathrm{a} \alpha} \Psi_{\beta}^{\mathrm{a}}-\frac{1}{4} \bar{W}_{\dot{\alpha} \dot{\beta}} \bar{\Psi}_{\mathrm{a}}^{\dot{\alpha}} \bar{\Psi}^{\dot{\beta} \mathrm{a}} \\
& +3 Y^{--r} D_{r}^{++}+\left(2 \lambda^{r \alpha-} \nabla_{\alpha}^{-} D_{r}^{++}+\text {h.c. }\right)
\end{aligned}
$$




$$
\begin{aligned}
& +\left(\frac{1}{4} W^{r}\left(\nabla^{-}\right)^{2} D_{r}^{++}-\frac{1}{2} W^{r} M_{\mathrm{a}}^{-} J_{r}^{\mathrm{a}+}+\text { h.c. }\right) \\
& +\left(\frac{3}{2} \chi^{\alpha+} D^{--} q_{\mathrm{b}}^{+} \Psi_{\alpha}^{\mathrm{b}}-\frac{3}{2} D_{\mathrm{v}}^{--}\left(\chi^{\alpha+} q_{\mathrm{b}}^{+} \Psi_{\alpha}^{\mathrm{b}}\right)+\text { h.c. }\right) .
\end{aligned}
$$

In arriving at this result, we have extensively used (6.36) and (6.37) and have written the fermion kinetic term with the analytic basis $\operatorname{Sp}(n)$ connection

$$
\widehat{\nabla}_{\alpha \dot{\alpha}} \bar{\Psi}^{\dot{\alpha} \mathrm{a}}=\nabla_{\alpha \dot{\alpha}} \bar{\Psi}^{\dot{\alpha} \mathrm{a}}+\nabla_{\alpha \dot{\alpha}} q^{\mathrm{c}+} \omega_{\mathrm{c}}^{-\mathrm{ab}} \bar{\Psi}_{\mathrm{b}}^{\dot{\alpha}}
$$

Now we can reconstruct the kinetic terms. The one for the physical fermions emerges after rewriting $\Psi_{\mathrm{a}}^{\alpha} \widehat{\nabla}_{\alpha \dot{\alpha}} \bar{\Psi}^{\dot{\alpha} \mathrm{a}}=\zeta_{\mathrm{a}}^{\alpha} \widehat{\nabla}_{\alpha \dot{\alpha}} \bar{\zeta}^{\dot{\alpha} \mathrm{a} a}$ where now $\widehat{\nabla}_{a}$ is in the central basis. To find the correct bosonic kinetic term, we use $\nabla_{\alpha \dot{\alpha}} q^{\mathrm{a}+}=\nabla_{\alpha \dot{\alpha}} \phi^{\mu} E_{\mu}{ }^{\mathrm{a}+}$ and $g_{\mu \nu}=2 E_{(\mu}{ }^{\mathrm{a}+} E_{\nu)}{ }^{-}$to give

$$
\begin{aligned}
-\frac{1}{4} q_{\mathrm{a}}^{+} \nabla^{\dot{\alpha} \alpha} A_{\alpha \dot{\alpha}}^{\mathrm{a}-} & =-\frac{1}{4} \nabla^{\dot{\alpha} \alpha}\left(q_{\mathrm{a}}^{+} A_{\alpha \dot{\alpha}}^{\mathrm{a}-}\right)-\frac{1}{4} \nabla^{a} \phi^{\mu} \nabla_{a} \phi^{\nu} g_{\mu \nu}+\frac{i}{4} \nabla^{\dot{\alpha} \alpha} q^{\mathrm{a}+} \Psi_{\alpha}^{\mathrm{b}} \Psi_{\dot{\alpha}}^{\mathrm{c}} \omega_{\mathrm{abc}}^{-} \\
& =\frac{1}{2} K_{\mu} \widehat{\nabla}^{a} \nabla_{a} \phi^{\mu}+\nabla^{\dot{\alpha} \alpha}\left(\frac{1}{4} K_{\mu} \nabla_{\alpha \dot{\alpha}} \phi^{\mu}-\frac{1}{4} q_{\mathrm{a}}^{+} A_{\alpha \dot{\alpha}}^{\mathrm{a}-}\right)+\frac{i}{4} \nabla^{\dot{\alpha} \alpha} q^{\mathrm{a}+} \Psi_{\alpha}^{\mathrm{b}} \Psi_{\dot{\alpha}}^{\mathrm{c}} \omega_{\mathrm{abc}}^{-},
\end{aligned}
$$

where $K_{\mu}=k_{\mathrm{D} \mu}=\partial_{\mu} K$ is the derivative of the hyperkähler potential. The covariant derivative $\widehat{\nabla}_{a}$ should be understood to also carry the target space affine connection. To simplify the remaining terms, we need the solution for $M_{\mathrm{a}}^{-}$and the expression for $\left(\nabla^{-}\right)^{2} D_{r}^{++} \mid$:

$$
\left(\nabla^{-}\right)^{2} D_{r}^{++} \mid=-4 \bar{W}^{s} f_{s r}{ }^{t} D_{t}^{+-}-2 \bar{W}^{s} J_{s}^{\mu} J_{r \mu}+\frac{1}{2} \zeta^{\mathrm{a}} \zeta^{\mathrm{b}} \mathcal{D}_{\mathrm{a} j} J_{r \mathrm{~b}}{ }^{j}
$$

where $D_{r}^{+-} \equiv D_{r}^{i j} v_{i}^{+} v_{j}^{-}=\frac{1}{2} D_{\mathrm{v}}^{--} D_{r}^{++}$. This leads to

$$
\begin{aligned}
T_{0}^{\prime}= & \frac{1}{2} K_{\mu} \widehat{\nabla}^{a} \nabla_{a} \phi^{\mu}+\left(\frac{i}{4} \zeta_{\mathrm{a}}^{\alpha} \widehat{\nabla}_{\alpha \dot{\alpha}} \bar{\zeta}^{\dot{\alpha} \mathrm{a}}+\text { h.c. }\right)+\frac{1}{16} R_{\mathrm{abcd}} \zeta^{\mathrm{a}} \zeta^{\mathrm{b}} \bar{\zeta}^{\mathrm{c}} \bar{\zeta}^{\mathrm{d}} \\
& +\left(\frac{1}{4} W^{r} \zeta^{\mathrm{a}} \zeta^{\mathrm{b}} \mathcal{D}_{\mathrm{a} j} J_{r \mathrm{~b}}{ }^{j}+2 \lambda^{r \alpha-} \Omega_{r \alpha}^{+}-\frac{1}{4} W^{\alpha \beta} \zeta_{\mathrm{a} \alpha} \zeta_{\beta}^{\mathrm{a}}+\text { h.c. }\right) \\
& -\frac{1}{2} W^{r} \bar{W}^{s} J_{s}^{\mu} J_{r \mu}+3 Y^{--r} D_{r}^{++}-\frac{3}{2} D K \\
& +\left(\frac{3}{2} \chi^{\alpha+} D_{\mathrm{v}}^{--} q_{\mathrm{b}}^{+} \Psi_{\alpha}^{\mathrm{b}}-\frac{3}{2} D_{\mathrm{v}}^{--}\left(\chi^{\alpha+} q_{\mathrm{b}}^{+} \Psi_{\alpha}^{\mathrm{b}}\right)+\text { h.c. }\right) \\
& +\nabla^{\dot{\alpha} \alpha}\left(\frac{1}{4} K_{\mu} \nabla_{\alpha \dot{\alpha}} \phi^{\mu}-\frac{1}{4} q_{\mathrm{a}}^{+} A_{\alpha \dot{\alpha}}^{\mathrm{a}-}\right) .
\end{aligned}
$$

Each of the expressions in the first three lines depends on the $v^{i \pm}$ harmonics and not on $w_{i}^{ \pm}$. Because we are integrating against the $\mathrm{SU}(2)_{\mathrm{v}} / \mathrm{U}(1)_{\mathrm{v}}$ measure $\mathcal{V}^{++} \wedge \mathcal{V}^{--}$, the naive rules of harmonic integration apply now for the $v_{i}^{ \pm}$harmonics, leading to

$$
\begin{aligned}
T_{0}^{\prime}= & {\left[\frac{1}{2} K_{\mu} \widehat{\nabla}^{a} \nabla_{a} \phi^{\mu}+\left(\frac{i}{4} \zeta_{\mathrm{a}}^{\alpha} \widehat{\nabla}_{\alpha \dot{\alpha}} \bar{\zeta}^{\dot{\alpha} \mathrm{a}}+\text { h.c. }\right)+\frac{1}{16} R_{\mathrm{abcd}} \zeta^{\mathrm{a}} \zeta^{\mathrm{b}} \bar{\zeta}^{\mathrm{c}} \bar{\zeta}^{\mathrm{d}}\right.} \\
& +\left(\frac{1}{4} W^{r} \zeta^{\mathrm{a}} \zeta^{\mathrm{b}} \mathcal{D}_{\mathrm{a} j} J_{r \mathrm{~b}}{ }^{j}-\lambda^{r \alpha}{ }_{i} \zeta_{\alpha \mathrm{a}} J_{r}^{\mathrm{a} i}-\frac{1}{4} W^{\alpha \beta} \zeta_{\mathrm{a} \alpha} \zeta_{\beta}^{\mathrm{a}}-\frac{3}{4} \chi_{i}^{\alpha} A_{\mathrm{b}}{ }^{i} \zeta_{\alpha}{ }^{\mathrm{b}}+\text { h.c. }\right) \\
& \left.-\frac{1}{2} W^{r} \bar{W}^{s} J_{s}^{\mu} J_{r \mu}+Y^{i j r} D_{r i j}-\frac{3}{2} D K\right]+\nabla^{\dot{\alpha} \alpha}\left(\frac{1}{4} K_{\mu} \nabla_{\alpha \dot{\alpha}} \phi^{\mu}-\frac{1}{4} q_{\mathrm{a}}^{+} A_{\alpha \dot{\alpha}}^{\mathrm{a}-}\right) .
\end{aligned}
$$


The bracketed terms will end up in the final Lagrangian. The next step is to integrate by parts the final term. We can swap the order of integration to yield

$$
\frac{i}{2 \pi} \int \mathrm{d}^{4} x e \nabla^{\dot{\alpha} \alpha} \int_{\mathcal{S}} \mathcal{V}^{++} \wedge \mathcal{V}^{--}\left(\frac{1}{4} \nabla_{\alpha \dot{\alpha}} K-\frac{1}{4} q_{\mathrm{a}}^{+} A_{\alpha \dot{\alpha}}^{\mathrm{a}-}\right)
$$

The term in parentheses actually vanishes at this order in its $\theta$-expansion because it can be rewritten as $\frac{i}{8} D_{\mathrm{v}}^{--} \nabla_{\alpha}^{+} \bar{\nabla}_{\dot{\alpha}}^{+} K$ where to this order $K$ is harmonic independent. This expression now vanishes using usual rules of harmonic integration. The lacunae in this argument is that the gravitino contributions lead to an $\mathcal{O}\left(\theta^{3}\right)$ term, which must be separately addressed. Denote these by $T_{0 \mid Q}^{\prime}$ :

$$
\begin{aligned}
T_{0 \mid Q}^{\prime}= & \frac{1}{4} \psi^{\dot{\alpha} \alpha \beta-} q_{\mathrm{b}}^{+} \nabla_{\beta \dot{\alpha}} \Psi_{\alpha}^{\mathrm{b}}+i \psi_{\alpha}^{\dot{\alpha} \alpha-} \bar{\lambda}_{\dot{\alpha}}^{r-} D_{r}^{++}+\frac{i}{4}\left(\psi_{m}^{-} \sigma^{m} \bar{\Psi}^{\mathrm{c}}\right) \bar{W}^{r} q_{\mathrm{b}}^{+} \partial_{\mathrm{c}+} \mathcal{J}_{r}^{\mathrm{b}+} \\
& -\frac{i}{2} \psi_{\alpha}^{\dot{\alpha} \alpha+} q_{\mathrm{b}}^{+} \Xi_{\dot{\alpha}}^{\mathrm{b}--}-\frac{1}{8} \psi^{\dot{\alpha} \alpha \beta+} \Psi_{\beta \mathrm{b}} A_{\alpha \dot{\alpha}}^{\mathrm{b}-}-\frac{1}{8} \psi_{j}^{\dot{\alpha} \alpha \beta} \nabla_{\alpha \dot{\alpha}} \nabla_{\beta}{ }^{j} K \mid \\
& -\frac{i}{8} \psi_{\alpha \dot{\alpha} j}{ }_{j}^{\alpha} \bar{W}_{\dot{\beta}}^{\dot{\alpha}} \bar{\zeta}^{\dot{\beta} \mathrm{a}} A_{\mathrm{a}}{ }^{j}+\frac{3 i}{8}\left(\psi_{m j} \sigma^{m} \bar{\chi}^{j}\right) K+\text { h.c. }
\end{aligned}
$$

Now we return to the explicit gravitino terms in (6.42). They can be written

$$
T_{1}^{(-2,2)}=-\frac{i}{2} D_{\mathrm{w}}^{++}\left(\left(\psi_{m}^{-} \sigma^{m}\right) \dot{\alpha} q_{\mathrm{b}}^{+} \Xi^{\dot{\alpha} \mathrm{b}--}-\frac{1}{8}\left(\psi_{m}^{-} \sigma^{m} \bar{\Psi}^{\mathrm{a}}\right) \Psi^{\mathrm{b}} \Psi^{\mathrm{c}} \omega_{\mathrm{abc}}^{-}\right)+\text {h.c. }
$$

where we imposed the equations of motion through $\mathcal{O}\left(\theta^{2}\right)$. Proceeding as in (6.45) gives

$$
\begin{aligned}
T_{1}^{\prime}= & \frac{i}{2}\left(\psi_{m}^{+} \sigma^{m}\right)_{\dot{\alpha}} q_{\mathrm{b}}^{+} \Xi^{\dot{\alpha} \mathrm{b}--}-\frac{i}{16}\left(\psi_{m}^{+} \sigma^{m} \bar{\Psi}^{\mathrm{a}}\right) \Psi^{\mathrm{b}} \Psi^{\mathrm{c}} \omega_{\mathrm{abc}}^{-} \\
& -\frac{1}{4}\left(\psi_{a}^{-} \sigma^{a} \bar{\sigma}^{b}\right)^{\beta} q_{\mathrm{b}}^{+} \nabla_{b} \Psi_{\beta}^{\mathrm{b}+}-\frac{i}{4}\left(\psi_{m}^{-} \sigma^{m}\right)_{\dot{\alpha}} \bar{W}^{\dot{\alpha}}{ }_{\dot{\beta}} q_{\mathrm{b}}^{+} \bar{\Psi}^{\dot{\beta} \mathrm{b}+}+\frac{3 i}{4}\left(\psi_{m}^{-} \sigma^{m} \bar{\chi}^{+}\right) K \\
& +2 i\left(\psi_{m}^{-} \sigma^{m} \bar{\lambda}^{r-}\right) D_{r}^{++}+\frac{i}{4}\left(\psi_{m}^{-} \sigma^{m} \bar{\Psi}^{\mathrm{c}}\right) q_{\mathrm{b}}^{+} \bar{W}^{r} \partial_{\mathrm{c}+\mathcal{J}_{r}^{\mathrm{b}+}+\text { h.c. }}
\end{aligned}
$$

Combining terms gives

$$
\begin{aligned}
T_{0 \mid Q}^{\prime}+T_{1}^{\prime}= & -\left(\psi_{a}^{-} \sigma^{a b}\right)^{\beta} q_{\mathrm{b}}^{+} \nabla_{b} \Psi_{\beta}^{\mathrm{b}+}+3 i\left(\psi_{m}^{-} \sigma^{m} \bar{\lambda}^{r-}\right) D_{r}^{++}-\frac{i}{2}\left(\psi_{m}^{-} \sigma^{m} \bar{\Psi}^{\mathrm{b}}\right) \bar{W}^{r} \mathcal{J}_{r \mathrm{~b}}{ }^{+} \\
& -\frac{1}{4} \psi^{\dot{\alpha} \alpha \beta-} \Psi_{\beta \mathrm{b}} \nabla_{\alpha \dot{\alpha}} q^{\mathrm{b}+}-\frac{1}{8} \psi^{\dot{\alpha} \alpha \beta}{ }_{j} \nabla_{\alpha \dot{\alpha}} \nabla_{\beta}{ }^{j} K-\frac{i}{8} \psi_{\alpha \dot{\alpha} j}{ }_{j}^{\alpha} \bar{W}^{\dot{\alpha}}{ }_{\dot{\beta}} \bar{\zeta}^{\dot{\beta} \mathrm{a}} A_{\mathrm{a}}{ }^{j} \\
& -\frac{i}{4}\left(\psi_{m}^{-} \sigma^{m}\right)_{\dot{\alpha}} \bar{W}^{\dot{\alpha}}{ }_{\dot{\beta}} q_{\mathrm{b}}^{+} \bar{\Psi}^{\dot{\beta} \mathrm{b}}+\frac{3 i}{8}\left(\psi_{m j} \sigma^{m} \bar{\chi}^{j}\right) K+\frac{3 i}{4}\left(\psi_{m}^{-} \sigma^{m} \bar{\chi}^{+}\right) K \\
& +D_{\mathrm{v}}^{++}\left[-\frac{1}{8} \psi^{\dot{\alpha} \alpha \beta-} \Psi_{\beta \mathrm{b}} A_{\alpha \dot{\alpha}}^{\mathrm{b}-}-\frac{i}{16}\left(\psi_{m}^{-} \sigma^{m} \bar{\Psi}^{\mathrm{a}}\right) \Psi^{\mathrm{b}} \Psi^{\mathrm{c}} \omega_{\mathrm{abc}}^{-}\right]+\text {h.c. }
\end{aligned}
$$

The argument of $D_{\mathrm{v}}^{++}$in the last line is actually independent of the $w_{i}^{ \pm}$harmonics, so it vanishes under the harmonic integral. Other terms can be simplified in analogous ways.

Putting everything together, the full component Lagrangian for the sigma model of a hyperkähler cone coupled to conformal supergravity is

$$
\mathcal{L}=\frac{1}{2} K_{\mu} \widehat{\square} \phi^{\mu}+\left(\frac{i}{4} \zeta_{\mathrm{a}}^{\alpha} \widehat{\nabla}_{\alpha \dot{\alpha}} \bar{\zeta}^{\dot{\alpha} \mathrm{a}}+\text { h.c. }\right)+\frac{1}{16} R_{\mathrm{abcd}} \zeta^{\mathrm{a}} \zeta^{\mathrm{b}} \bar{\zeta}^{\mathrm{c}} \bar{\zeta}^{\mathrm{d}}
$$




$$
\begin{aligned}
& +Y^{i j r} D_{r i j}+\lambda_{i}^{r \alpha} \zeta_{\alpha}^{\mathrm{a}} J_{r \mathrm{a}}{ }^{i}-\lambda_{\dot{\alpha}}^{r}{ }^{i} \bar{\zeta}_{\mathrm{a}}^{\dot{\alpha}} J_{r}{ }_{i}^{\mathrm{a}}-\frac{1}{2} W^{r} \bar{W}^{s} J_{s}^{\mu} J_{r \mu} \\
& +\frac{1}{4} W^{r} \zeta^{\mathrm{a}} \zeta^{\mathrm{b}} \mathcal{D}_{\mathrm{a} j} J_{r \mathrm{~b}}{ }^{j}+\frac{1}{4} \bar{W}^{r} \bar{\zeta}^{\mathrm{a}} \bar{\zeta}^{\mathrm{b}} \mathcal{D}_{\mathrm{a} j} J_{r \mathrm{~b}}{ }^{j} \\
& -\frac{3}{2} D K-\frac{1}{4}\left(W^{\alpha \beta} \zeta_{\mathrm{a} \alpha} \zeta_{\beta}^{\mathrm{a}}+\bar{W}_{\dot{\alpha} \dot{\beta}} \bar{\zeta}_{\mathrm{a}}^{\dot{\alpha}} \bar{\zeta}^{\dot{\beta} \mathrm{a}}\right)-\frac{3}{4}\left(\chi^{\alpha}{ }_{i} \zeta_{\alpha}^{\mathrm{b}} A_{\mathrm{b}}{ }^{i}-\bar{\chi}_{\dot{\alpha}}{ }^{i} \bar{\zeta}_{\mathrm{b}}^{\dot{\alpha}} A^{\mathrm{b}}{ }_{i}\right) \\
& +\left(\psi_{m j} \sigma^{m}\right)_{\dot{\alpha}}\left(\frac{1}{4} \widehat{\nabla}^{\dot{\alpha} \alpha} \zeta_{\alpha \mathrm{b}} A^{\mathrm{b} j}-\frac{i}{4} \bar{W}^{\dot{\alpha}} \bar{\beta}^{\dot{\beta}}{ }^{\dot{\beta} \mathrm{b}} A_{\mathrm{b}}{ }^{j}+\frac{3 i}{4} \bar{\chi}^{\dot{\alpha} j} K-\frac{i}{4} \bar{\zeta}^{\dot{\alpha} \mathrm{b}} \bar{W}^{r} J_{r \mathrm{~b}}{ }^{j}+i \bar{\lambda}_{k}^{\dot{\alpha} r} D_{r}{ }^{j k}\right) \\
& +\left(\bar{\psi}_{m}^{j} \bar{\sigma}^{m}\right)^{\alpha}\left(\frac{1}{4} \widehat{\nabla}_{\alpha \dot{\alpha}} \bar{\zeta}_{\mathrm{b}}^{\dot{\alpha}} A^{\mathrm{b}}{ }_{j}-\frac{i}{4} W_{\alpha}{ }^{\beta} \zeta_{\beta \mathrm{b}} A^{\mathrm{b}}{ }_{j}+\frac{3 i}{4} \chi_{\alpha j} K-\frac{i}{4} \zeta_{\alpha \mathrm{b}} W^{r} J_{r j}^{\mathrm{b}}-i \lambda_{\alpha r}^{k} D_{r j k}\right) .
\end{aligned}
$$

This agrees with the same result derived in projective superspace [10] and can be compared with the original component reference [44].

\section{Further applications and outlook}

The main goal of this paper has been to construct a covariant formulation of harmonic superspace based on the geometry of conformal superspace. This included (i) the construction of invariant actions in a general gauge; (ii) the specification of how to pass covariantly between full harmonic and analytic superspaces; and (iii) the explicit component reduction formula in the central basis. One important task which we have not pursued is the complete specification of the vielbeins and connections in the analytic basis in terms of the prepotentials. This was the main result of $[25,26]$. Although that approach used a different superspace (equivalent to conformal supergravity coupled to vector and non-linear multiplet compensators), one could follow essentially identical steps within conformal superspace to arrive at comparable results. The reverse is also true: within the framework of $[25,26]$, one could derive e.g. the explicit component reduction rule in the central basis. We have sketched how this could be accomplished by introducing compensated derivatives. The explicit expressions are expected to be substantially more complicated due to the presence of additional torsion tensors, including a dimension-1/2 spinor superfield. Of course, when the action in question is superconformal, all dependence on the compensators must drop out of the final component action; the advantage of conformal superspace is that such extraneous objects are avoided from the beginning, leading to vastly simpler computations.

Although we have chosen to limit the scope of this investigation to the above goal and the sample calculation in section 6 , a number of applications become immediately apparent. In the remainder of this concluding section, we will briefly sketch several proposals.

\section{Projective superspace and prepotentials}

One particular aspect we have not explored is the full connection between the curved harmonic and projective superspaces, which was in part a driving motivation of this research. Certainly in the central basis the mapping sketched in the introduction between complex harmonic and projective superfields must hold. A more interesting question is how to use this mapping to convert harmonic supergravity prepotentials into projective ones. For gauge prepotentials, this was sketched by Jain and Siegel [33]. The analytic 
gauge prepotential $V$ in projective superspace is given by [5]

$$
e^{V}=e^{-V_{S}} e^{V_{N}}
$$

in terms of two bridge superfields. ${ }^{18} V_{N}$ is the arctic multiplet bridge, converting analytic arctic multiplets into covariant ones, while $V_{S}$ is the antarctic bridge. $V_{N}$ is well-defined near the north pole and $V_{S}$ near the south pole, but neither is itself analytic. Using the mapping between harmonic and projective multiplets, $V_{N}$ and $V_{S}$ can be written in terms of the complex harmonic bridge $\mathcal{B}$ as

$$
V_{N}(v)=\left.i \mathcal{B}(v, w)\right|_{w_{i}^{-}=(1,0)}, \quad V_{S}(v)=\left.i \mathcal{B}(v, w)\right|_{w_{i}^{-}=(0,1)} .
$$

It is easy to confirm that while $V_{N}$ and $V_{S}$ are not themselves analytic, the combination (7.1) is and transforms appropriately under the arctic and antarctic $\lambda$-groups. Extending this idea to the supergravity prepotentials would seem to be reasonably straightforward. For example, the harmonic bridges for the coordinates should similarly decompose into separate arctic and antarctic bridges. However, a more explicit investigation is warranted.

\section{Quaternion-Kähler superspace}

One of the many successes of harmonic superspace is its very elegant description of general hypermultiplet systems, including supergravity-matter couplings. Rigid harmonic superspace admits general off-shell hypermultiplet actions, with the harmonic potential encoding the sigma model geometry. In a similar way, curved harmonic superspace admits off-shell hypermultiplet actions, with the potential function(s) encoding the sigma model [38, 52, 63].

There are two existing formulations. The first, following the same conventions as in section 6, involves the Lagrangian

$$
\mathscr{L}^{(2,2)}=\frac{1}{2} Q_{\mathrm{a}}^{+} \nabla_{\mathrm{w}}^{++} Q^{\mathrm{a}+}+H^{(2,2)}\left(Q^{+}, w^{-}\right)
$$

with superfields $Q^{\mathrm{a}+}$ with a $=1, \cdots, 2(n+1)$ describing the sigma model of a $4(n+1)$ dimensional hyperkähler cone coupled to conformal supergravity. Each of the superfields has Weyl weight one and charge $(1,0)$ under $\mathrm{U}(1)_{\mathrm{v}} \times \mathrm{U}(1)_{\mathrm{w}}$. As is well-known, a $4(n+$ 1)-dimensional hyperkähler cone is in one-to-one correspondence with a $4 n$-dimensional quaternion-Kähler manifold [68] (see also [69]). This structure can be made more apparent in superspace, which leads to the second formulation [63]. One makes the field redefinition $Q^{\mathrm{a}+} \rightarrow\left(\omega \mathcal{Q}^{\mathrm{a}+}, q^{i+}\right)$, where the superfields $q^{i+}$ have Weyl weight one and charge $(1,0)$, $\mathcal{Q}^{\mathrm{a}+}, \mathrm{a}=1, \cdots, 2 n$, have vanishing Weyl weight and charge $(0,1)$, and $\omega:=q^{i+} w_{i}^{-}$. The Lagrangian becomes

$$
\mathscr{L}^{(2,2)}=\frac{1}{2} q_{i}^{+} \nabla_{\mathrm{w}}^{++} q^{i+}+\omega^{2}\left[\frac{1}{2} \mathcal{Q}_{\mathrm{a}}^{+} \nabla_{\mathrm{w}}^{++} \mathcal{Q}^{\mathrm{a}+}+H^{+4}\left(\mathcal{Q}^{+}, q^{i+} / \omega, w_{i}^{-}\right)\right] .
$$

The term within square braces has charge $(0,4)$ and vanishing Weyl weight. The hypermultiplet $q^{i+}$ is interpreted as a compensating multiplet while $\mathcal{Q}^{\text {a+ }}$ are the matter multiplets.

\footnotetext{
${ }^{18}$ See also [12] and [11] for recent discussions in curved 3D and 5D projective superspace.
} 
This is equivalent (up to a gauge choice and a sign change in the compensator kinetic term) to the original form given in [52]. It was shown in [38] that the general quaternion-Kähler sigma model is described by two prepotentials $\mathcal{L}_{\mathrm{a}}{ }^{+}$and $\mathcal{L}^{+4}$, and the action above is just the special gauge $\mathcal{L}_{\mathrm{a}}^{+}=\mathcal{Q}_{\mathrm{a}}^{+}$(with $\mathcal{L}^{+4} \rightarrow H^{+4}$ ) of this general form.

The two expressions (7.3) and (7.4) bear a striking similarity to analogous formulae involving chiral superfields in $\mathcal{N}=1$ superspace. There the action for a $2(n+1)$-dimensional Kähler cone sigma model coupled to conformal supergravity involves the superspace Lagrangian

$$
\mathscr{L}=K\left(\Phi^{\mathrm{a}}, \bar{\Phi}^{\overline{\mathrm{a}}}\right)
$$

with chiral superfields $\Phi^{\mathrm{a}}$ for a $=1, \cdots, n+1$. The function $K$ is the Kähler potential for a Kähler cone. Reorganizing the chiral superfields as $\Phi^{\mathrm{a}} \rightarrow\left(\phi \varphi^{\mathrm{a}}, \phi\right)$ where $\phi$ has Weyl weight one and $\varphi^{\mathrm{a}}, \mathrm{a}=1, \cdots, n$ are weight zero, the Lagrangian can be rewritten

$$
\mathscr{L}=-3 \phi \bar{\phi} e^{-\mathcal{K} / 3}
$$

where $\mathcal{K}$ is the potential for a $2 n$-dimensional Hodge-Kähler manifold. The fields $\varphi^{\mathrm{a}}$ are matter fields and $\phi$ is a chiral compensator. The usual factor of 3 is chosen for convenience and the negative sign is consistent with the role of $\phi$ as a compensator.

The $\mathcal{N}=1$ action actually has a third form, which is in many applications more useful. Recall that the component reduction of (7.6) still requires an inconvenient set of super-Weyl gauge choices to be imposed to canonically normalize the graviton and gravitino actions (see e.g. the discussion in [70]). This can be directly addressed at the superfield level by absorbing the Hodge-Kähler potential into the superspace vielbein $[71,72]$. Equivalently, one can interpret $\mathcal{K}$ as if it were the prepotential for some additional $\mathrm{U}(1)_{\mathcal{K}}$ symmetry under which the compensator $\phi$ is charged. Moving to covariantly chiral superfields $\phi$, the Lagrangian simplifies to

$$
\mathcal{L}=-3 \phi \bar{\phi} .
$$

Now the matter fields are encoded within the composite $\mathrm{U}(1)_{\mathcal{K}}$ connection, and the matter action appears within the composite auxiliary field $D$ associated with $\mathrm{U}(1)_{\mathcal{K}}$. Adopting the gauge $\phi=1$ fixes the Weyl gauge and a linear combination of $\mathrm{U}(1)_{R}$ and $\mathrm{U}(1)_{\mathcal{K}}$, leaving another linear combination as the composite Kähler U(1) symmetry of the physical action. This geometrizes the Kähler potential, ensuring that the $\mathrm{U}(1)_{R}$ gauge field of supergravity is identified with the composite $U(1)$ potential of the Kähler line bundle while also canonically normalizing all terms in the component action. The resulting superspace is known as Kähler superspace [71, 72] and provides a general framework for handling $\mathcal{N}=1$ supergravity-matter systems, even including higher-derivative couplings in a Kähler covariant form. For example, a simple class of such higher-derivative terms is given by a full superspace integral of

$$
\mathscr{L}=c_{1} R \bar{R}+c_{2} G^{a} G_{a}+c_{3} \mathcal{K}^{\alpha \dot{\alpha}} \mathcal{K}_{\alpha \dot{\alpha}}
$$

for real constants $c_{i}$, where $R$ and $G_{a}$ are the torsion superfields of Kähler superspace and $\mathcal{K}_{\alpha \dot{\alpha}}=\mathcal{K}_{\mathrm{a} \overline{\mathrm{b}}} \mathcal{D}_{\alpha} \varphi^{\mathrm{a}} \overline{\mathcal{D}}_{\dot{\alpha}} \bar{\varphi}^{\overline{\mathrm{b}}}$. Writing such Kähler-covariant terms in the original superspace frame is significantly more complicated. 
In light of these observations, it is natural to conjecture a third formulation of $\mathcal{N}=2$ superspace corresponding to a slight reformulation of (7.4), just as (7.7) reformulates (7.6). The idea is that (7.4) can be rewritten

$$
\mathscr{L}^{(2,2)}=\frac{1}{2} q_{i}^{+}\left(\nabla_{\mathrm{w}}^{++} q^{i+}-V_{\mathrm{w}}{ }^{i}{ }^{j+} q^{j+}\right)
$$

where $V_{\mathrm{w}}{ }^{i}$ is a composite prepotential for an additional "matter" $\mathrm{SU}(2)$ group rotating the $q^{i+}$; this is known as the Pauli-Gürsey group $\mathrm{SU}(2)_{\mathrm{PG}}[2]$. In the central basis for $\mathrm{SU}(2)_{\mathrm{PG}}$, the equations of motion set $q^{i+}$ to be a covariant $\mathcal{O}(1)$ multiplet, $q^{i+}=f^{i}{ }_{j} v^{j+}$. The Weyl gauge along with a linear combination of $\mathrm{SU}(2)_{R}$ and $\mathrm{SU}(2)_{\mathrm{PG}}$ is fixed by taking $f^{i}{ }_{j}=\delta^{i}{ }_{j}$, and the other linear combination of $\mathrm{SU}(2)_{R}$ and $\mathrm{SU}(2)_{\mathrm{PG}}$ survives as the composite $\mathrm{SU}(2)$ symmetry of the quaternion-Kähler manifold. This strongly suggests that one can construct a quaternion-Kähler superspace as the $\mathcal{N}=2$ analogue of Kähler superspace. We intend to explore this subject in the near future.

\section{Higher-derivative terms}

The advantage of a covariant approach is the ease of component reductions, including all couplings to supergravity. We demonstrated this by deriving the general two-derivative hyperkähler cone action, but it would be plausible to address higher-derivative actions as well. Large classes of these have been discussed recently in projective superspace [73] and comparable calculations could undoubtedly be pursued within the harmonic approach (see e.g. the rigid higher-derivative terms of [74]).

If indeed one can construct a quaternion-Kähler superspace as the $\mathcal{N}=2$ analogue of Kähler superspace, it would undoubtedly provide the natural framework for addressing higher-derivative terms involving hypermultiplets. For example, in the gauge-fixed formulation of $\mathcal{N}=2$ superspace, there exist torsion superfields $S^{i j}$ and $G_{a}{ }^{i j}$. It is plausible that from these one could construct a higher-derivative harmonic superspace Lagrangian analogous to (7.8), corresponding to a new curvature-squared invariant.

It was shown recently in [75] that a certain Ricci-squared invariant could be constructed, given by the chiral superspace Lagrangian, $\mathscr{L}_{c}=\frac{1}{6} \overline{\mathcal{D}}^{i j} \bar{S}_{i j}+\bar{S}^{i j} \bar{S}_{i j}+\bar{Y}_{\dot{\alpha} \dot{\beta}} \bar{Y}^{\dot{\alpha} \dot{\beta}}$, in $\mathrm{SU}(2)$ superspace. This is compensator-independent and, when combined with the known Weyl-squared invariant, gives the $\mathcal{N}=2$ Gauss-Bonnet. Compensator-dependent higher-derivative invariants have been constructed e.g. using the trick of building composite vector multiplets out of fundamental tensor multiplets $[49,73,76,77]$. Because a tensor multiplet is dual to a general $Q^{+}$hypermultiplet, it is possible that such terms may be constructed for general quaternionic-Kähler manifolds; if so, one might be able to construct the $\mathcal{N}=2$ analogue of one of the $\mathcal{N}=1$ invariants (7.8). Its form in the proposed quaternion-Kähler superspace might be particularly elegant.

\section{Supergravity prepotentials and higher derivative terms}

Finally, we should mention that one important application of having both a covariant form of harmonic superspace as well as the analytic basis prepotentials is that one could more easily find harmonic-independent prepotentials. It was shown in [78] that the fundamental 
scalar prepotential $\mathcal{H}$ of $\mathcal{N}=2$ conformal supergravity could be uncovered in this way, and one could analyze how it appears within the central-basis superspace vielbeins, mirroring the harmonic construction of [26]. This would be useful e.g. for understanding the supercurrents of higher-derivative Lagrangians such as the ones discussed above.

\section{Acknowledgments}

I would like to thank Sergei Kuzenko, Gabriele Tartaglino-Mazzucchelli, and Evgeny Ivanov for valuable comments on the draft. This work was supported in part by the ERC Advanced Grant no. 246974, "Supersymmetry: a window to non-perturbative physics" and by the European Commission Marie Curie International Incoming Fellowship grant no. PIIF-GA-2012-627976.

\section{A Analytic integrals and densities}

This appendix is a summary and continuation of appendix B of [10], which addressed covariant integration over supermanifolds. Let us recall the basics. A supermanifold $\mathcal{M}$ (without boundary) of dimension $D$ possesses local coordinates $z^{M}, M=1, \ldots, D$, a vielbein $E_{M}{ }^{A}$, and separate connection $H_{M}$ a associated with internal symmetries, which we denote $\mathcal{H}$. Under diffeomorphisms both connections transform as one-forms, while under $\mathcal{H}$-gauge transformations,

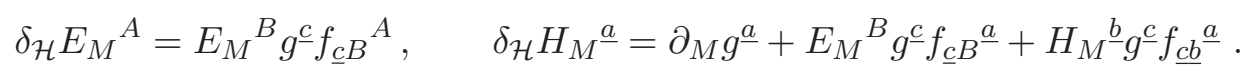

The parameters $f$ are structure constants of a soft algebra including the covariant curvatures associated with the vielbein and $\mathcal{H}$-connection (see e.g. the discussion in [45]). When diffeomorphisms are covariantized with the $\mathcal{H}$-connection, the full transformation rules become

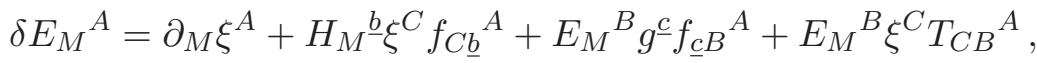

$$
\begin{aligned}
& \delta H_{M^{\underline{a}}}=\partial_{M} g^{\underline{a}}+E_{M}^{B} g^{\underline{c}} f_{\underline{c} B^{\underline{a}}}+H_{M^{\underline{b}}}^{\underline{\underline{b}}} g^{\underline{c}} f_{\underline{c} \underline{\underline{b}}}^{\underline{a}}+H_{M^{\underline{b}} \xi^{C}} f_{C \underline{\underline{b}}}^{\underline{a}}+E_{M}{ }^{B} \xi^{C} R_{C B^{\underline{a}}} \text {. }
\end{aligned}
$$

An action over the full supermanifold, $\int \mathrm{d}^{D} z E \mathscr{L}$, is invariant provided $\mathscr{L}$ is a scalar under diffeomorphisms and transforms under $\mathcal{H}$ as $\delta_{\mathcal{H}} \mathscr{L}=-(-)^{A} g^{\underline{b}} f_{\underline{b} A}{ }^{A} \mathscr{L}$.

\section{A.1 Analytic submanifolds}

We are interested in an analytic submanifold $\mathfrak{M}$ (without boundary) of dimension $d$ with local coordinates $\mathfrak{z}^{m}, m=1, \ldots, d$. We have in mind a situation where the original coordinates can be decomposed as $z^{M}=\left(\mathfrak{z}^{m}, y^{\mu}\right)$ with the submanifold $\mathfrak{M}$ corresponding to the surface parametrized by $\mathfrak{z}^{m}$ with (for example) $y^{\mu}=0$. The coordinates $\mathfrak{z}^{m}$ and $y^{\mu}$ may be bosonic or fermionic; we denote the grading of a coordinate $z^{M}$ by $(-)^{M}$. The vielbein and its inverse are given by

$$
E_{M}^{A}=\left(\begin{array}{cc}
\mathcal{E}_{m}{ }^{a} & E_{m}{ }^{\alpha} \\
E_{\mu}{ }^{a} & E_{\mu}{ }^{\alpha}
\end{array}\right), \quad E_{A}{ }^{M}=\left(\begin{array}{cc}
E_{a}{ }^{m} & E_{a}{ }^{\mu} \\
E_{\alpha}{ }^{m} & \phi_{\alpha}{ }^{\mu}
\end{array}\right),
$$


with the assumption that both $\mathcal{E}_{m}{ }^{a}$ and $\phi_{\alpha}{ }^{\mu}$ are invertible, with inverses $\mathcal{E}_{a}{ }^{m}$ and $\phi_{\mu}{ }^{\alpha}$, respectively. This allows one to compactly specify all the remaining components of the vielbein and its inverse in terms of these quantities, $E_{m}{ }^{\alpha}$, and $E_{\alpha}{ }^{m}$ :

$$
\begin{aligned}
& E_{M}{ }^{A}=\left(\begin{array}{c|c}
\mathcal{E}_{m}{ }^{a} & E_{m}{ }^{\alpha} \\
\hline-\phi_{\mu}{ }^{\beta} E_{\beta}{ }^{n} \mathcal{E}_{n}{ }^{a} \phi_{\mu}{ }^{\alpha}-\phi_{\mu}{ }^{\beta} E_{\beta}{ }^{n} E_{n}{ }^{\alpha}
\end{array}\right), \\
& E_{A}{ }^{M}=\left(\begin{array}{c|c}
\mathcal{E}_{a}{ }^{m}-\mathcal{E}_{a}{ }^{n} E_{n}{ }^{\beta} E_{\beta}{ }^{m} & -\mathcal{E}_{a}{ }^{n} E_{n}{ }^{\beta} \phi_{\beta}{ }^{\mu} \\
\hline E_{\alpha}{ }^{m} & \phi_{\alpha}{ }^{\mu}
\end{array}\right) .
\end{aligned}
$$

No assumptions have been made about $E_{m}{ }^{\alpha}$ or $E_{\alpha}{ }^{m}$. We treat $\mathcal{E}_{m}{ }^{a}$ as the vielbein of the submanifold $\mathfrak{M}$. In particular, the class of diffeomorphisms acting on $\mathfrak{z}^{m}$ induce

$$
\delta \mathcal{E}_{m}{ }^{a}=\partial_{m} \xi^{n} \mathcal{E}_{n}{ }^{a}+\xi^{n} \partial_{n} \mathcal{E}_{m}{ }^{a}, \quad \xi^{M}=\left(\xi^{m}, 0\right)
$$

as required for a vielbein. This formula holds even if $\xi^{m}$ depends on $y^{\mu}$.

The submanifold is an analytic submanifold (although not yet in the analytic basis) if the following properties are satisfied. Under $\mathcal{H}$-gauge transformations and covariant diffeomorphisms generated by $\xi^{A}=\left(0, \xi^{\alpha}\right)$, the analytic vielbein transforms into itself via

$$
\delta \mathcal{E}_{m}{ }^{a}=\mathcal{E}_{m}{ }^{b} \xi^{\gamma} T_{\gamma b}{ }^{a}+\mathcal{E}_{m}{ }^{b} g^{\underline{c}} f_{\underline{c} b}{ }^{a}, \quad \xi^{A}=\left(0, \xi^{\alpha}\right) .
$$

These conditions derive from the transformation rules of $E_{M}{ }^{A}$ assuming the vanishing of the torsion tensor $T_{\gamma \beta}{ }^{a}$ and the structure constants $f_{\underline{c} \beta}{ }^{a}$, which permit the existence of superfields annihilated by $\nabla_{\alpha}$. It is convenient to decompose the full set of possible transformations into the diffeomorphisms on $\mathfrak{M}$ with $\xi^{M}=\left(\xi^{m}, 0\right)$, the covariant diffeomorphisms generated by $\xi^{A}=\left(0, \xi^{\alpha}\right)$, and the $\mathcal{H}$-gauge transformations. This is always possible to do using the invertibility of $\phi_{\alpha}{ }^{\mu}$ and $\mathcal{E}_{m}{ }^{a}$.

We will not actually require that all analytic superfields be annihilated by each of the $\nabla_{\alpha}$. We have in mind the situation where some of the $\nabla_{\alpha}$ have an interpretation as charge generators. In harmonic superspace, these would be $\nabla_{\mathrm{v}}^{0}$ and $\nabla_{\mathrm{w}}^{0}$. So we instead call an analytic superfield $\Psi$ one for which

$$
\nabla_{\alpha} \Psi=c_{\alpha}^{(\Psi)} \Psi
$$

where $c_{\alpha}^{(\Psi)}$ is a (possibly vanishing) constant number. Only bosonic covariant derivatives may possess non-vanishing $c_{\alpha}^{(\Psi)}$.

Suppose now we have a scalar Lagrangian $\mathcal{L}$ that obeys

$$
\nabla_{\alpha} \mathcal{L}=-(-)^{b} T_{\alpha b}{ }^{b} \mathcal{L} \equiv c_{\alpha}^{(\mathcal{L})} \mathcal{L}, \quad \delta_{\mathcal{H}} \mathcal{L}=-(-)^{a} g^{\underline{\underline{b}}} f_{\underline{b} a}{ }^{a} \mathcal{L} .
$$

where the expression $T_{\alpha b}{ }^{b}(-)^{b}$ built from the torsion tensor is constant (and possibly vanishing). We may define an analytic action $\mathcal{S}$ over the submanifold $\mathfrak{M}$,

$$
\mathcal{S}=\int \mathrm{d}^{d} \mathfrak{z} \mathcal{E} \mathcal{L}, \quad \mathcal{E}=\operatorname{sdet} \mathcal{E}_{m}{ }^{a}
$$

Using (A.5) and (A.6), one finds that under a general transformation parametrized as $\delta=\xi^{m} \partial_{m}+\xi^{\alpha} \nabla_{\alpha}+\delta_{\mathcal{H}}$, the integrand of (A.9) transforms as a total derivative, $\delta(\mathcal{E} \mathcal{L})=$ 
$\partial_{m}\left(\xi^{m} \mathcal{E} \mathcal{L}\right)$ so the action is invariant. In particular, the action (A.9) is invariant even under diffeomorphisms in $y^{\mu}$. These can be interpreted as arbitrary small deformations of the embedding of $\mathfrak{M}$ in $\mathcal{M}$. In other words, the particular choice of the embedding has no effect on the action integral.

As an example of a covariant action principle, let us take harmonic superspace on the analytic submanifold. We group the derivatives as

$$
\nabla_{a}=\left(\nabla_{a}, \nabla_{\underline{\alpha}}^{-}, \nabla_{\mathrm{v}}^{--}, \nabla_{\mathrm{w}}^{++}\right), \quad \nabla_{\alpha}=\left(\nabla_{\underline{\alpha}}^{+}, \nabla_{\mathrm{v}}^{++}, \nabla_{\mathrm{w}}^{--}, \nabla_{\mathrm{v}}^{0}, \nabla_{\mathrm{w}}^{0}\right),
$$

with the coordinates $\mathfrak{z}^{m}=\left(x^{m}, \theta^{\underline{\mu}}+\zeta, \tilde{\zeta}\right)$ parametrizing the submanifold $\mathfrak{M}=\mathcal{M}^{4 \mid 4} \times \mathcal{S}$. From the torsion constraints, the covariant Lagrangian $\mathcal{L}$ must obey

$$
\nabla_{\underline{\alpha}}^{+} \mathcal{L}=\nabla_{\mathrm{v}}^{++} \mathcal{L}=\nabla_{\mathrm{w}}^{--} \mathcal{L}=0, \quad \nabla_{\mathrm{v}}^{0} \mathcal{L}=\nabla_{\mathrm{w}}^{0} \mathcal{L}=2
$$

The measure $\mathcal{E}$ in turn transforms under covariant $\xi^{\alpha}$ diffeomorphisms as

$$
\delta \mathcal{E}=-2\left(\xi_{\mathrm{v}}^{0}+\xi_{\mathrm{w}}^{0}\right) \mathcal{E} .
$$

For these reasons we denote the Lagrangian and measure by $\mathscr{L}^{(2,2)}$ and $E^{(-2,-2)}$, respectively. The covariant action is then just (4.5).

Actually, even "full" harmonic superspace is an analytic superspace in a sense, as we always restrict to twisted biholomorphic quantities of fixed $\mathrm{U}(1)_{\mathrm{v}} \times \mathrm{U}(1)_{\mathrm{w}}$ charge. Now the decomposition of derivatives is

$$
\nabla_{a}=\left(\nabla_{a}, \nabla_{\underline{\alpha}}^{ \pm}, \nabla_{\mathrm{v}}^{--}, \nabla_{\mathrm{w}}^{++}\right), \quad \nabla_{\alpha}=\left(\nabla_{\mathrm{v}}^{++}, \nabla_{\mathrm{w}}^{--}, \nabla_{\mathrm{v}}^{0}, \nabla_{\mathrm{w}}^{0}\right),
$$

with the coordinates $\mathfrak{z}^{m}=\left(x^{m}, \theta^{\mu \pm}, \zeta, \tilde{\zeta}\right)$ on $\mathcal{M}^{4 \mid 8} \times \mathcal{S}$. Lagrangians obey

$$
\nabla_{\mathrm{v}}^{++} \mathcal{L}=\nabla_{\mathrm{w}}^{--} \mathcal{L}=0, \quad \nabla_{\mathrm{v}}^{0} \mathcal{L}=-2 \mathcal{L}, \quad \nabla_{\mathrm{w}}^{0} \mathcal{L}=2 \mathcal{L} .
$$

The measure $\mathcal{E}$ transforms under covariant $\xi^{\alpha}$ diffeomorphisms as

$$
\delta \mathcal{E}=2\left(\xi_{\mathrm{v}}^{0}-\xi_{\mathrm{w}}^{0}\right) \mathcal{E} .
$$

For these reasons we denote the Lagrangian and measure by $\mathscr{L}^{(-2,2)}$ and $E^{(+2,-2)}$, respectively. The covariant action is then just (4.2).

\section{A.2 Analytic gauge, densities and transformation rules}

Now let us make a special choice for the embedding. Suppose we can adopt a basis $\hat{\mathfrak{z}}^{m}$ for the analytic coordinates where

$$
\nabla_{\alpha} \hat{\mathfrak{z}}^{m}=c_{\alpha}^{(m)} \hat{\mathfrak{z}}^{m}
$$

for some (possibly vanishing) bosonic constants $c_{\alpha}^{(m)}$. We will call this an analytic coordinate system, and it is equivalent to requiring $\hat{E}_{\alpha}{ }^{m}=c_{\alpha}^{(m)} \hat{\mathfrak{z}}^{m}$. Consistency requires

$$
\nabla_{\beta} \hat{E}_{\alpha}{ }^{m}=c_{\beta}^{(m)} c_{\alpha}^{(m)} \hat{\mathfrak{z}}^{m}, \quad \nabla_{b} \hat{E}_{\alpha}{ }^{m}=c_{\alpha}^{(m)} \hat{E}_{b}{ }^{m}, \quad \delta_{\mathcal{H}} \hat{E}_{\alpha}{ }^{m}=-g^{\underline{b}} f_{\underline{b} \alpha}{ }^{\beta} \hat{E}_{\beta}{ }^{m}=0 .
$$


We will further assume that the other coordinates $\hat{y}^{\mu}$ have been chosen so that $\phi_{\alpha}{ }^{\mu}=$ $\phi_{\alpha}{ }^{\mu}(y)$ is independent of $\mathfrak{z}^{m}$; this is possible using the vanishing of the torsion tensor $T_{\alpha \beta}{ }^{c}$ and applying Frobenius' theorem. Now an analytic diffeomorphism is defined as a diffeomorphism preserving the above conditions. This leads to

$$
\delta^{*} \hat{\mathfrak{z}}^{m}=-\xi^{m}(\hat{\mathfrak{z}}), \quad \nabla_{\alpha} \hat{\xi}^{m}=c_{\alpha}^{(n)} \hat{\mathfrak{z}}^{n} \partial_{n} \hat{\xi}^{m}=c_{\alpha}^{(m)} \hat{\xi}^{m} .
$$

This condition ensures that the $\nabla_{\alpha}$ charge of the analytic parameter $\hat{\xi}^{m}$ matches that of the coordinate $\hat{\mathfrak{z}}^{m}$.

Using these assumptions, one may show that the analytic measure $\mathcal{E}$ is analytic in the sense of (A.7),

$$
\nabla_{\alpha} \mathcal{E}=\left(T_{\alpha b}^{b}(-)^{b}-\sum_{m} c_{\alpha}^{(m)}(-)^{m}\right) \mathcal{E}
$$

Now it is easy to show that the Lagrangian density, $\hat{\mathcal{L}}=\mathcal{E} \mathcal{L}$ is analytic, obeying

$$
\nabla_{\alpha} \hat{\mathcal{L}}=-\sum_{m} c_{\alpha}^{(m)}(-)^{m} \hat{\mathcal{L}}
$$

It may perhaps be useful to illustrate these points using the analytic harmonic superspace as an example. We choose the analytic basis coordinates $\hat{\mathfrak{z}}^{m}=\left(\hat{x}^{m}, \hat{\theta}^{\mu+}, \hat{u}_{i}^{ \pm}\right)$. We can choose each of these to be annihilated by $\nabla_{\underline{\alpha}}^{+}, \nabla_{\mathrm{v}}^{++}, \nabla_{\mathrm{w}}^{--}$, and $\nabla_{\mathrm{v}}^{0}$, while

$$
\nabla_{\mathrm{w}}^{0} \hat{x}^{m}=0, \quad \nabla_{\mathrm{w}}^{0} \hat{\theta}^{\underline{\mu}}=\hat{\theta}^{\underline{\mu}}, \quad \nabla_{\mathrm{w}}^{0} \hat{u}_{i}^{ \pm}= \pm \hat{u}_{i}^{ \pm} .
$$

Now one can show, in contrast with (A.12), that

$$
\nabla_{\mathrm{v}}^{0} \mathcal{E}=-2 \mathcal{E}, \quad \nabla_{\mathrm{w}}^{0} \mathcal{E}=+2 \mathcal{E}
$$

and so the Lagrangian density $\hat{\mathcal{L}}$ now obeys

$$
\nabla_{\mathrm{v}}^{0} \hat{\mathcal{L}}=0, \quad \nabla_{\mathrm{w}}^{0} \hat{\mathcal{L}}=4 \hat{\mathcal{L}} .
$$

In a similar way, we can adopt an "analytic basis" for full harmonic superspace: the simplest choice is actually the central basis! Now we have $\mathfrak{z}^{m}=\left(x^{m}, \theta^{\underline{\mu}}{ }_{\imath}, v^{i+}, w_{i}^{-}\right)$, where each is annihilated by $\nabla_{\mathrm{v}}^{++}$and $\nabla_{\mathrm{w}}^{--}$. We easily see that $\mathcal{E}$ is independent of the harmonics - in fact, it is just the Berezinian sdet $E_{M}{ }^{A}$ in the central basis - and the action integral becomes (4.1). From this perspective, the measure factor $\mathcal{V}^{++} \wedge \mathcal{W}^{--}$is just the anholonomic measure associated with the constrained coordinates $v^{i+}$ and $w_{i}^{-}$.

\section{A.3 Rules for total derivatives}

We will need some general rules for integrating total derivatives. It is a simple exercise to show that for the full supermanifold $\mathcal{M}$,

$$
\int \mathrm{d}^{D} z E \nabla_{A} \mathcal{V}^{A}(-)^{A}=\int \mathrm{d}^{D} z\left[\nabla_{M}\left(E \mathcal{V}^{A} E_{A}{ }^{M}\right)(-)^{M}-E \mathcal{V}^{A} T_{A B}{ }^{B}(-)^{B}\right] .
$$

The term involving $\nabla_{M}$ may be decomposed into $\partial_{M}$, which may be discarded, and a connection piece. The connection piece may be non-trivial as $E \mathcal{V}^{A} E_{A}{ }^{M}$ might transform 
under some of the $\mathcal{H}$ group. We are really interested in integrals on the analytic submanifold $\mathfrak{M}$, where

$$
\begin{aligned}
\int \mathrm{d}^{d} \mathfrak{z} \mathcal{E} \nabla_{a} \mathcal{V}^{a}(-)^{a}=\int & \mathrm{d}^{d} \mathfrak{z}\left[\nabla_{m}\left(\mathcal{E} \mathcal{V}^{a} \mathcal{E}_{a}{ }^{m}\right)(-)^{m}-\mathcal{E} \mathcal{V}^{a} T_{a b}{ }^{b}(-)^{b}\right. \\
& \left.-\mathcal{E} \mathcal{E}_{a}{ }^{m} E_{m}{ }^{\alpha}\left(\nabla_{\alpha} \mathcal{V}^{a}+T_{\alpha b}{ }^{b} \mathcal{V}^{a}(-)^{b}+\mathcal{V}^{b} T_{b \alpha}{ }^{a}\right)(-)^{a}\right] .
\end{aligned}
$$

Again $\nabla_{m}$ may be decomposed into $\partial_{m}$, which may be discarded, and a connection piece.

The expression (A.25) can be a bit unwieldy, so a few examples should help. Let us take full harmonic superspace, which is the analytic superspace of twisted biholomorphic fields with fixed charges. We choose the non-vanishing components of $\mathcal{V}^{A}$ to be $\mathcal{V}^{w^{--}}$ and $\mathcal{V}^{\mathrm{v}++}$. Keeping in mind that the Lagrangian in this case must carry charge $(-2,2)$, these components may be labeled $\mathcal{V}^{(-2,0)}$ and $\mathcal{V}^{(0,2)}$ (up to a sign). The Lagrangian is $\mathscr{L}^{(-2,2)}=\nabla_{\mathrm{w}}^{++} \mathcal{V}^{(-2,0)}+\nabla_{\mathrm{v}}^{--} \mathcal{V}^{(0,2)}$. These are both valid integrands provided $\mathcal{V}^{(-2,0)}$ and $\mathcal{V}^{(0,2)}$ are twisted biholomorphic and invariant with respect to the gauge symmetries. Using (A.25), it is easy to show that this is a total derivative.

As another example, we take analytic superspace and choose a single non-vanishing component $\mathcal{V}^{\mathrm{w}--}$. Because the Lagrangian now must have charge $(2,2)$, this component may be labeled $\mathcal{V}^{(2,0)}$. The Lagrangian $\mathscr{L}^{(2,2)}=\nabla_{\mathrm{w}}^{++} \mathcal{V}^{(2,0)}$ is covariant provided $\mathcal{V}^{(2,0)}$ is a twisted biholomorphic analytic primary. It is easy to check this is a total derivative. In contrast, the expression $\nabla_{\mathrm{v}}^{--} \mathcal{V}^{(4,2)}$ is not even generically a covariant Lagrangian because it is not annihilated by $\nabla_{\mathrm{v}}^{++}$.

Open Access. This article is distributed under the terms of the Creative Commons Attribution License (CC-BY 4.0), which permits any use, distribution and reproduction in any medium, provided the original author(s) and source are credited.

\section{References}

[1] A. Galperin, E. Ivanov, S. Kalitsyn, V. Ogievetsky and E. Sokatchev, Unconstrained $N=2$ matter, Yang-Mills and supergravity theories in harmonic superspace, Class. Quant. Grav. 1 (1984) 469 [Erratum ibid. 2 (1985) 127] [INSPIRE].

[2] A. Galperin, E. Ivanov, V. Ogievetsky and E. Sokatchev, Harmonic superspace, Cambridge University Press, Cambridge U.K. (2001).

[3] A. Karlhede, U. Lindström and M. Roček, Selfinteracting tensor multiplets in $N=2$ superspace, Phys. Lett. B 147 (1984) 297 [InSPIRE].

[4] U. Lindström and M. Roček, New hyper-Kähler metrics and new supermultiplets, Commun. Math. Phys. 115 (1988) 21 [INSPIRE].

[5] U. Lindström and M. Roček, $N=2$ super Yang-Mills theory in projective superspace, Commun. Math. Phys. 128 (1990) 191 [INSPIRE].

[6] U. Lindström and M. Roček, Properties of hyper-Kähler manifolds and their twistor spaces, Commun. Math. Phys. 293 (2010) 257 [arXiv:0807.1366] [InSPIRE].

[7] S.M. Kuzenko, Lectures on nonlinear $\sigma$-models in projective superspace, J. Phys. A 43 (2010) 443001 [arXiv:1004.0880] [INSPIRE]. 
[8] P.S. Howe and G.G. Hartwell, A superspace survey, Class. Quant. Grav. 12 (1995) 1823 [INSPIRE].

[9] G.G. Hartwell and P.S. Howe, $(N, p, q)$ harmonic superspace, Int. J. Mod. Phys. A 10 (1995) 3901 [hep-th/9412147] [INSPIRE].

[10] D. Butter, New approach to curved projective superspace, Phys. Rev. D 92 (2015) 085004 [arXiv: 1406.6235] [INSPIRE].

[11] D. Butter, S.M. Kuzenko, J. Novak and G. Tartaglino-Mazzucchelli, Conformal supergravity in five dimensions: new approach and applications, JHEP 02 (2015) 111 [arXiv:1410.8682] [INSPIRE].

[12] S.M. Kuzenko and G. Tartaglino-Mazzucchelli, $N=4$ supersymmetric Yang-Mills theories in $A d S_{3}, J H E P 05$ (2014) 018 [arXiv: 1402.3961] [INSPIRE].

[13] F. Gonzalez-Rey, M. Roček, S. Wiles, U. Lindström and R. von Unge, Feynman rules in $N=2$ projective superspace: 1 . Massless hypermultiplets, Nucl. Phys. B 516 (1998) 426 [hep-th/9710250] [INSPIRE].

[14] F. Gonzalez-Rey and R. von Unge, Feynman rules in $N=2$ projective superspace. 2. Massive hypermultiplets, Nucl. Phys. B 516 (1998) 449 [hep-th/9711135] [INSPIRE].

[15] F. Gonzalez-Rey, Feynman rules in $N=2$ projective superspace. 3. Yang-Mills multiplet, hep-th/9712128 [INSPIRE].

[16] D. Jain and W. Siegel, On projective hoops: loops in hyperspace, Phys. Rev. D 83 (2011) 105024 [arXiv: 1012.3758] [INSPIRE].

[17] D. Jain and W. Siegel, Improved methods for hypergraphs, Phys. Rev. D 88 (2013) 025018 [arXiv: 1302.3277] [INSPIRE].

[18] S.M. Kuzenko and G. Tartaglino-Mazzucchelli, Five-dimensional superfield supergravity, Phys. Lett. B 661 (2008) 42 [arXiv:0710.3440] [INSPIRE].

[19] S.M. Kuzenko and G. Tartaglino-Mazzucchelli, 5D supergravity and projective superspace, JHEP 02 (2008) 004 [arXiv:0712.3102] [INSPIRE].

[20] S.M. Kuzenko and G. Tartaglino-Mazzucchelli, Super-Weyl invariance in $5 D$ supergravity, JHEP 04 (2008) 032 [arXiv:0802.3953] [INSPIRE].

[21] S.M. Kuzenko, U. Lindström, M. Roček and G. Tartaglino-Mazzucchelli, $4 D N=2$ supergravity and projective superspace, JHEP 09 (2008) 051 [arXiv:0805.4683] [INSPIRE].

[22] S.M. Kuzenko, U. Lindström, M. Roček and G. Tartaglino-Mazzucchelli, On conformal supergravity and projective superspace, JHEP 08 (2009) 023 [arXiv:0905.0063] [INSPIRE].

[23] S.M. Kuzenko and G. Tartaglino-Mazzucchelli, Different representations for the action principle in $4 D N=2$ supergravity, JHEP 04 (2009) 007 [arXiv:0812.3464] [INSPIRE].

[24] S.M. Kuzenko, On superconformal projective hypermultiplets, JHEP 12 (2007) 010 [arXiv:0710.1479] [INSPIRE].

[25] B. Delamotte and J. Kaplan, The geometry of $N=2$ supergravity in harmonic superspace, Class. Quant. Grav. 4 (1987) 1223 [INSPIRE].

[26] A.S. Galperin, N.A. Ky and E. Sokatchev, $N=2$ supergravity in superspace: solution to the constraints, Class. Quant. Grav. 4 (1987) 1235 [INSPIRE]. 
[27] D. Butter, Projective multiplets and hyper-Kähler cones in conformal supergravity, JHEP 06 (2015) 161 [arXiv: 1410.3604] [INSPIRE].

[28] D. Butter and J. Novak, Component reduction in $N=2$ supergravity: the vector, tensor and vector-tensor multiplets, JHEP 05 (2012) 115 [arXiv:1201.5431] [INSPIRE].

[29] E. Ivanov and G. Valent, Quaternionic metrics from harmonic superspace: Lagrangian approach and quotient construction, Nucl. Phys. B 576 (2000) 543 [hep-th/0001165] [INSPIRE].

[30] D. Butter, S.M. Kuzenko, J. Novak and G. Tartaglino-Mazzucchelli, Conformal supergravity in three dimensions: off-shell actions, JHEP 10 (2013) 073 [arXiv: 1306.1205] [INSPIRE].

[31] S.M. Kuzenko, J. Novak and G. Tartaglino-Mazzucchelli, $N=6$ superconformal gravity in three dimensions from superspace, JHEP 01 (2014) 121 [arXiv:1308.5552] [INSPIRE].

[32] M. Nishimura and Y. Tanii, $N=6$ conformal supergravity in three dimensions, JHEP 10 (2013) 123 [arXiv:1308.3960] [INSPIRE].

[33] D. Jain and W. Siegel, Deriving projective hyperspace from harmonic, Phys. Rev. D 80 (2009) 045024 [arXiv:0903.3588] [INSPIRE].

[34] E.T. Newman, Gauge theories, the holonomy operator and the Riemann-Hilbert problem, J. Math. Phys. 27 (1986) 2797 [inSPIRE].

[35] S.M. Kuzenko, Projective superspace as a double punctured harmonic superspace, Int. J. Mod. Phys. A 14 (1999) 1737 [hep-th/9806147] [inSPIRE].

[36] D. Butter, Relating harmonic and projective descriptions of $N=2$ nonlinear $\sigma$-models, JHEP 11 (2012) 120 [arXiv:1206.3939] [INSPIRE].

[37] A. Galperin, E. Ivanov, V. Ogievetsky and E. Sokatchev, Conformal invariance in harmonic superspace, in Quantum field theory and quantum statistics, I.A. Batalin, C.J. Isham and G.A. Vilkovisky eds., vol. 2, Adam Hilger, Bristol U.K. (1987), pg. 233.

[38] A. Galperin, E. Ivanov and O. Ogievetsky, Harmonic space and quaternionic manifolds, Annals Phys. 230 (1994) 201 [hep-th/9212155] [INSPIRE].

[39] S.J. Gates, Jr., C.M. Hull and M. Roček, Twisted multiplets and new supersymmetric nonlinear $\sigma$-models, Nucl. Phys. B 248 (1984) 157 [INSPIRE].

[40] T. Buscher, U. Lindström and M. Roček, New supersymmetric $\sigma$ models with Wess-Zumino terms, Phys. Lett. B 202 (1988) 94 [InSPIRE].

[41] U. Lindström, I.T. Ivanov and M. Roček, New $N=4$ superfields and $\sigma$-models, Phys. Lett. B 328 (1994) 49 [hep-th/9401091] [INSPIRE].

[42] G. Tartaglino-Mazzucchelli, $2 D N=(4,4)$ superspace supergravity and bi-projective superfields, JHEP 04 (2010) 034 [arXiv:0911.2546] [INSPIRE].

[43] S.M. Kuzenko, U. Lindström and G. Tartaglino-Mazzucchelli, Off-shell supergravity-matter couplings in three dimensions, JHEP 03 (2011) 120 [arXiv:1101.4013] [INSPIRE].

[44] B. de Wit, B. Kleijn and S. Vandoren, Superconformal hypermultiplets, Nucl. Phys. B 568 (2000) 475 [hep-th/9909228] [INSPIRE].

[45] D. Butter, $N=2$ conformal superspace in four dimensions, JHEP 10 (2011) 030 [arXiv:1103.5914] [INSPIRE]. 
[46] B. de Wit, J.W. van Holten and A. Van Proeyen, Structure of $N=2$ supergravity, Nucl. Phys. B 184 (1981) 77 [Erratum ibid. B 222 (1983) 516] [inSPIRE].

[47] S.V. Ketov, B.B. Lokhvitsky and I.V. Tyutin, Hyper-Kähler $\sigma$ models in extended superspace, Theor. Math. Phys. 71 (1987) 496 [Teor. Mat. Fiz. 71 (1987) 226] [InSPIRE].

[48] S.V. Ketov and B.B. Lokhvitsky, Some generalizations of $N=2$ Yang-Mills matter couplings, Class. Quant. Grav. 4 (1987) L137 [InSPIRE].

[49] B. de Wit, R. Philippe and A. Van Proeyen, The improved tensor multiplet in $N=2$ supergravity, Nucl. Phys. B 219 (1983) 143 [INSPIRE].

[50] E.S. Fradkin and M.A. Vasiliev, Minimal set of auxiliary fields in $\mathrm{SO}(2)$ extended supergravity, Phys. Lett. B 85 (1979) 47 [INSPIRE].

[51] B. de Wit and J.W. van Holten, Multiplets of linearized $\mathrm{SO}(2)$ supergravity, Nucl. Phys. B 155 (1979) 530 [inSPIRE].

[52] A.S. Galperin, E.A. Ivanov, V.I. Ogievetsky and E. Sokatchev, $N=2$ supergravity in superspace: different versions and matter couplings, Class. Quant. Grav. 4 (1987) 1255 [INSPIRE].

[53] R. Grimm, Solution of the Bianchi identities in $\mathrm{SU}(2)$ extended superspace with constraints, in Unification of the fundamental particle interactions, S. Ferrara, J. Ellis and P. van Nieuvenhuizen eds., Springer U.S.A. (1980), pg. 509.

[54] A. Galperin and V. Ogievetsky, $N=2 D=4$ supersymmetric $\sigma$-models and Hamiltonian mechanics, Class. Quant. Grav. 8 (1991) 1757 [InSPIRE].

[55] S.V. Ketov, Superconformal hypermultiplets in superspace, Nucl. Phys. B 582 (2000) 95 [hep-th/0001109] [INSPIRE].

[56] A. Galperin, E. Ivanov and V. Ogievetsky, Superspace actions and duality transformations for $N=2$ tensor multiplets, Phys. Scr. T 15 (1987) 176.

[57] S.M. Kuzenko, On $N=2$ supergravity and projective superspace: dual formulations, Nucl. Phys. B 810 (2009) 135 [arXiv:0807.3381] [InSPIRE].

[58] D. Butter and S.M. Kuzenko, A dual formulation of supergravity-matter theories, Nucl. Phys. B 854 (2012) 1 [arXiv:1106.3038] [INSPIRE].

[59] P. Breitenlohner and M.F. Sohnius, Superfields, auxiliary fields and tensor calculus for $N=2$ extended supergravity, Nucl. Phys. B 165 (1980) 483 [INSPIRE].

[60] S.J. Gates, Jr., Supercovariant derivatives, super Weyl groups and $N=2$ supergravity, Nucl. Phys. B 176 (1980) 397 [inSPIRE].

[61] A. Galperin, E. Ivanov, V. Ogievetsky and E. Sokatchev, Hyper-Kähler metrics and harmonic superspace, Commun. Math. Phys. 103 (1986) 515 [INSPIRE].

[62] A.S. Galperin, E.A. Ivanov, V.I. Ogievetsky and E. Sokatchev, Gauge field geometry from complex and harmonic analyticities. Hyper-Kähler case, Annals Phys. 185 (1988) 22 [INSPIRE].

[63] J.A. Bagger, A.S. Galperin, E.A. Ivanov and V.I. Ogievetsky, Gauging $N=2 \sigma$ models in harmonic superspace, Nucl. Phys. B 303 (1988) 522 [INSPIRE].

[64] C.M. Hull, A. Karlhede, U. Lindström and M. Roček, Nonlinear $\sigma$ models and their gauging in and out of superspace, Nucl. Phys. B 266 (1986) 1 [INSPIRE]. 
[65] G. Sierra and P.K. Townsend, The hyper-Kähler supersymmetric $\sigma$ model in six-dimensions, Phys. Lett. B 124 (1983) 497 [INSPIRE].

[66] G. Sierra and P.K. Townsend, The gauge invariant $N=2$ supersymmetric $\sigma$ model with general scalar potential, Nucl. Phys. B 233 (1984) 289 [INSPIRE].

[67] J. Bagger and E. Witten, Matter couplings in $N=2$ supergravity, Nucl. Phys. B 222 (1983) 1 [INSPIRE].

[68] A. Swann, Hyper-Kähler and quaternionic Kähler geometry, Math. Ann. 289 (1991) 421.

[69] B. de Wit, M. Roček and S. Vandoren, Gauging isometries on hyper-Kähler cones and quaternion Kähler manifolds, Phys. Lett. B 511 (2001) 302 [hep-th/0104215] [INSPIRE].

[70] J. Wess and J. Bagger, Supersymmetry and supergravity, Princeton Univ. Pr., Princeton U.S.A. (1992).

[71] P. Binétruy, G. Girardi, R. Grimm and M. Müller, Kähler transformations and the coupling of matter and Yang-Mills fields to supergravity, Phys. Lett. B 189 (1987) 83 [INSPIRE].

[72] P. Binétruy, G. Girardi and R. Grimm, Supergravity couplings: a geometric formulation, Phys. Rept. 343 (2001) 255 [hep-th/0005225] [INSPIRE].

[73] D. Butter and S.M. Kuzenko, New higher-derivative couplings in $4 D N=2$ supergravity, JHEP 03 (2011) 047 [arXiv: 1012.5153] [INSPIRE].

[74] P.C. Argyres, A.M. Awad, G.A. Braun and F.P. Esposito, Higher derivative terms in $N=2$ supersymmetric effective actions, JHEP 07 (2003) 060 [hep-th/0306118] [INSPIRE].

[75] D. Butter, B. de Wit, S.M. Kuzenko and I. Lodato, New higher-derivative invariants in $N=2$ supergravity and the Gauss-Bonnet term, JHEP 12 (2013) 062 [arXiv:1307.6546] [INSPIRE].

[76] W. Siegel, Off-shell $N=2$ supersymmetry for the massive scalar multiplet, Phys. Lett. B 122 (1983) 361 [inSPIRE].

[77] B. de Wit and F. Saueressig, Off-shell $N=2$ tensor supermultiplets, JHEP 09 (2006) 062 [hep-th/0606148] [INSPIRE].

[78] S.M. Kuzenko and S. Theisen, Correlation functions of conserved currents in $N=2$ superconformal theory, Class. Quant. Grav. 17 (2000) 665 [hep-th/9907107] [INSPIRE]. 